\title{
Analysis of broad-lined Type Ic supernovae from the (intermediate) Palomar Transient Factory
}

\author{
F. Taddia ${ }^{1}$, J. Sollerman ${ }^{1}$, C. Fremling ${ }^{2}$, C. Barbarino ${ }^{1}$, E. Karamehmetoglu ${ }^{1}$, I. Arcavi ${ }^{3}$, S. B. Cenko ${ }^{4,5}$, \\ A. V. Filippenko ${ }^{6,7}$, A. Gal-Yam ${ }^{8}$, D. Hiramatsu ${ }^{9,10}$, G. Hosseinzadeh ${ }^{11}$, D. A. Howell ${ }^{9,10}$, S. R. Kulkarni ${ }^{2}$, \\ R. Laher ${ }^{12}$, R. Lunnan ${ }^{1,2}$, F. Masci ${ }^{12}$, P. E. Nugent ${ }^{6,13}$, A. Nyholm ${ }^{1}$, D. A. Perley ${ }^{14}$, \\ R. Quimby ${ }^{15,16}$, and J. M. Silverman ${ }^{6,17}$
}

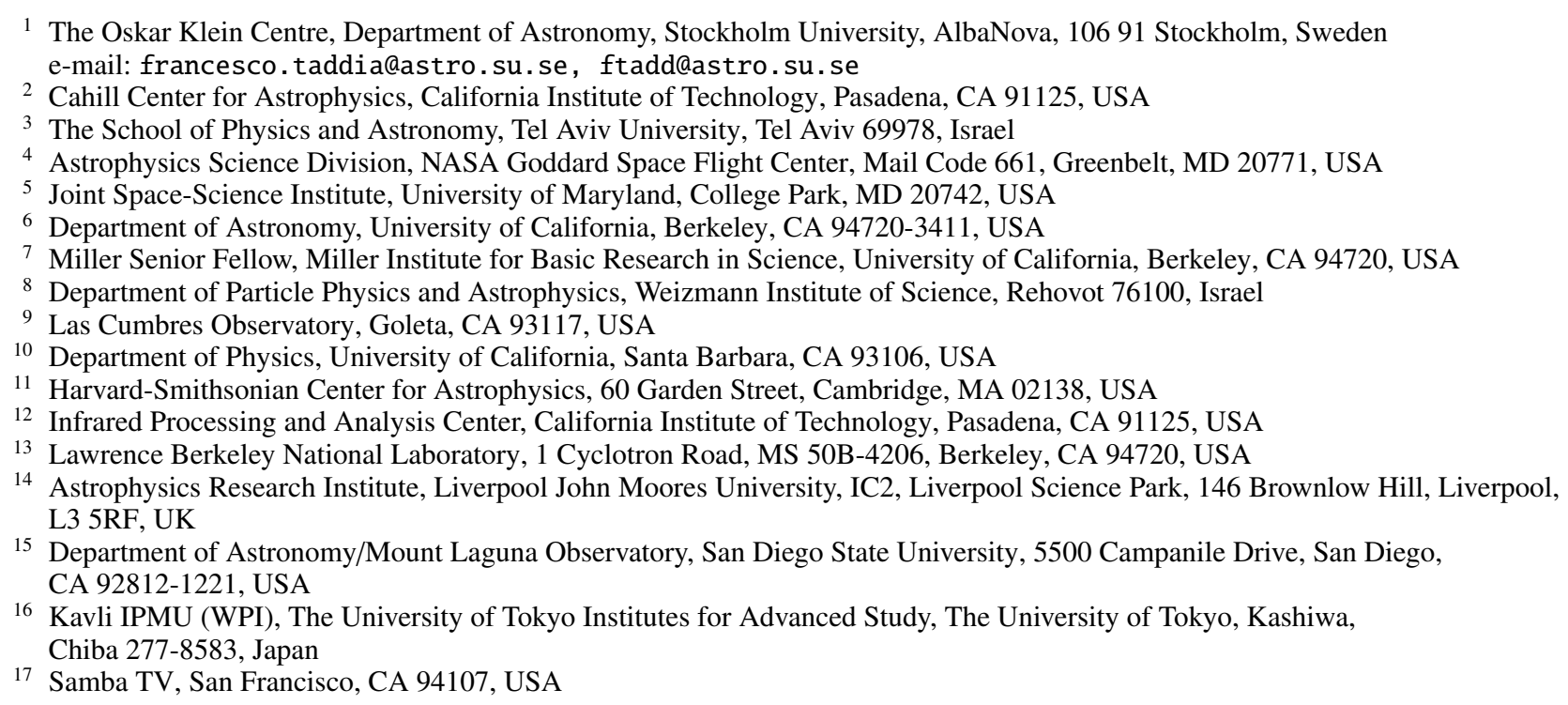

Received 15 October 2018 / Accepted 12 November 2018

\begin{abstract}
We study 34 Type Ic supernovae that have broad spectral features (SNe Ic-BL). This is the only SN type found in association with longduration gamma-ray bursts (GRBs). We obtained our photometric data with the Palomar Transient Factory (PTF) and its continuation, the intermediate PTF (iPTF). This is the first large, homogeneous sample of SNe Ic-BL from an untargeted survey. Furthermore, given the high observational cadence of iPTF, most of these SNe Ic-BL were discovered soon after explosion. We present K-corrected Bgriz light curves of these $\mathrm{SNe}$, obtained through photometry on template-subtracted images. We analyzed the shape of the $r$-band light curves, finding a correlation between the decline parameter $\Delta m_{15}$ and the rise parameter $\Delta m_{-10}$. We studied the SN colors and, based on $g-r$, we estimated the host-galaxy extinction for each event. Peak $r$-band absolute magnitudes have an average of $-18.6 \pm 0.5$ mag. We fit each $r$-band light curve with that of SN 1998bw (scaled and stretched) to derive the explosion epochs. We computed the bolometric light curves using bolometric corrections, $r$-band data, and $g-r$ colors. Expansion velocities from Fe II were obtained by fitting spectral templates of SNe Ic. Bolometric light curves and velocities at peak were fitted using the semianalytic Arnett model to estimate ejecta mass $M_{\mathrm{ej}}$, explosion energy $E_{K}$ and ${ }^{56} \mathrm{Ni}$ mass $M\left({ }^{56} \mathrm{Ni}\right)$ for each $\mathrm{SN}$. We find average values of $M_{\mathrm{ej}}=4 \pm 3 M_{\odot}$, $E_{K}=(7 \pm 6) \times 10^{51} \mathrm{erg}$, and $M\left({ }^{56} \mathrm{Ni}\right)=0.31 \pm 0.16 M_{\odot}$. The parameter distributions were compared to those presented in the literature and are overall in agreement with them. We also estimated the degree of ${ }^{56} \mathrm{Ni}$ mixing using scaling relations derived from hydrodynamical models and we find that all the SNe are strongly mixed. The derived explosion parameters imply that at least $21 \%$ of the progenitors of SNe Ic-BL are compatible with massive (>28 $M_{\odot}$ ), possibly single stars, whereas at least $64 \%$ might come from less massive stars in close binary systems.
\end{abstract}

Key words. supernovae: general

\section{Introduction}

Core-collapse supernovae (CC SNe) are explosions of massive $\left(M_{\text {ZAMS }} \gtrsim 8 M_{\odot}\right)$ stars. So-called stripped-envelope (SE) SNe which show deficiency or lack of $\mathrm{H}$, or even $\mathrm{He}$, and are hence classified as $\mathrm{SNe} \mathrm{IIb} / \mathrm{Ib}$ or Ic - can be found among CC SNe (e.g.,
Filippenko 1997, for a review). These SE SNe could arise from single massive $\left(M_{\text {ZAMS }} \gtrsim 30 M_{\odot}\right.$ ) stars stripped of their $\mathrm{H} / \mathrm{He}$ envelopes by strong stellar winds (Conti 1976), or they could be the explosions of somewhat less massive stars $\left(M_{\text {ZAMS }} \lesssim 20 M_{\odot}\right)$ stripped by their companions in binary systems (e.g., Yoon et al. 2010; Lyman et al. 2016; Taddia et al. 2018a). 
Among He-poor SNe Ic, there are events characterized by broad lines in their spectra, indicating particularly fast expansion velocities. These $\mathrm{SNe}$ are called broad-lined $\mathrm{SNe}$ Ic, or SNe Ic-BL. As shown by Modjaz et al. (2016), normal SNe Ic have Fe II velocities of $\sim 10^{4} \mathrm{~km} \mathrm{~s}^{-1}$ at light-curve peak, whereas $\mathrm{SNe}$ Ic-BL show Fe II velocities of $\sim(1.5-3) \times 10^{4} \mathrm{~km} \mathrm{~s}^{-1}$. In some cases, however, it is not straightforward to distinguish between normal SNe Ic and SNe Ic-BL, as there are SNe, such as SN 2004aw (Taubenberger et al. 2006), showing intermediate properties.

$\mathrm{SNe}$ Ic-BL is the only ${ }^{1} \mathrm{SN}$ class found to occur in correspondence with long-duration gamma-ray bursts (GRBs; Woosley \& Bloom 2006), the first clear example being SN 1998bw (Galama et al. 1998). These GRB-SNe are believed to be accompanied by the launch of collimated relativistic outflows, as shown in the case of SN 1998bw, whose strong radio emission revealed relativistic ejecta (Kulkarni et al. 1998). On the other hand, many SNe Ic-BL seem not to be associated with any GRB (see, e.g., SN 2002ap; Berger et al. 2002). Even SN 2012ap (Milisavljevic et al. 2015) and SN 2009bb (Pignata et al. 2011), which show evidence for a central engine and ejecta with relativistic velocities, are not coincident with a GRB. The reason for the difference between SNe Ic-BL with and without a GRB is still debated. It has been suggested that some $\mathrm{SNe}$ Ic-BL are accompanied by an off-axis GRB, which may be initially invisible but later should emerge in radio emission. However, this has never been observed (Soderberg et al. 2010). Corsi et al. (2016) estimated that $\langle 85 \%$ of their 15 SNe Ic-BL from the (intermediate) Palomar Transient Factory with radio observations could hide off-axis GRBs expanding in media with relatively high densities.

The light curves of SNe Ic-BL rise faster than those of $\mathrm{SNe} \mathrm{Ib}$ or IIb, and their peak magnitudes are brighter than those of the other SE SNe (e.g., Cano 2013; Taddia et al. 2015, 2018a; Lyman et al. 2016; Prentice et al. 2016). The modeling of the light curves has suggested that SNe Ic-BL eject larger ${ }^{56} \mathrm{Ni}$ masses and have higher explosion energies $\left(\sim 10^{52} \mathrm{erg}\right)$ than the other SE SN types (e.g., Cano 2013; Taddia et al. 2015, 2018a; Lyman et al. 2016; Prentice et al. 2016), but that the ejecta masses are not very different. Given the high explosion energies of these $\mathrm{SNe}$, they are sometimes also called "hypernovae". SNe Ic-BL and GRB-SNe have been suggested (also by host galaxy studies) to have progenitors that are younger and more massive than those of normal SNe Ic (e.g., Sanders et al. 2012; Cano 2013).

With the exception of the small SN Ic-BL sample from SDSS-II studied by Taddia et al. (2015), the other analyzed samples (e.g., Cano 2013; Lyman et al. 2016; Prentice et al. 2016) come from literature collections. Most of those SNe Ic-BL were discovered by targeted surveys, and the nonuniform data collections were obtained with many different telescopes. In addition, light curves of 10 SNe Ic-BL from the Center for Astrophysics (CfA) survey were released by Bianco et al. (2014), and Modjaz et al. (2016) performed a detailed analysis of the spectra of $10 \mathrm{SNe}$ Ic-BL without GRBs and $11 \mathrm{SNe}$ Ic-BL with GRBs.

Thanks to the Palomar Transient Factory (PTF; Rau et al. 2009; Law et al. 2009) and its continuation the intermediate PTF (iPTF; Kulkarni 2013), we can present a large (34 objects) sample of SNe Ic-BL with optical observations from an untargeted

\footnotetext{
1 With the exceptions of the spectroscopically normal SNe Ic 20021t and 2013ez (Cano et al. 2014) and the SLSN Ic 2011kl associated with the UL-GRB 111209A (Greiner et al. 2015).
}

survey. Almost all of the photometry has been obtained with two telescopes and reduced in the same way. This allows for a systematic study of the properties of this SN population and its progenitor stars.

This paper is structured as follows. In Sect. 2, we describe the basic information about our sample of SNe Ic-BL from the PTF+iPTF surveys. The SN observations and data reduction methods are presented in Sect. 3. The light curves in different bands are presented and analyzed (in particular, the $r$ band) in Sect. 4, as are the SN colors (especially $g-r$ ). These colors are also used to derive the host galaxy extinction. In Sect. 5 we present the SN spectra, whereas in Sect. 6 we produce and discuss the bolometric properties of our $\mathrm{SNe}$. By modeling the derived bolometric properties, in Sect. 7 we obtain progenitor and explosion parameters for our SNe. A discussion of the results (which are compared with those in the literature) and final conclusions are given in Sects. 8 and 9, respectively.

\section{The SN sample}

Our study includes 34 events discovered and monitored by PTF and iPTF in the years 2009-2017 that we classified as SNe IcBL. Their names and coordinates are reported in Table 1. These 34 events are a significant part of the total SE SN sample from PTF and iPTF, which counts 204 events, including SNe IIb (58), SN Ib (38), spectroscopically normal SNe Ic (60), and SNe Ibc (14). Supernovae Ibc are objects with spectra showing possible traces of $\mathrm{He}$ in their spectra, making them intermediate cases between spectroscopically normal SNe Ic and Ib. The SNe Ic and $\mathrm{SNe}$ Ib light curves will be studied by Barbarino et al. (in prep.), and the spectra have been presented and analyzed by Fremling et al. (2018). We do not consider superluminous Type I SNe (SLSNe I) in our study; these are typically characterized by absolute peak magnitudes $\lesssim-21 \mathrm{mag}$, long rise times, and peculiar spectral features at early times (Quimby et al. 2011; Gal-Yam 2012; De Cia et al. 2018; Lunnan et al. 2018).

The inclusion of SNe in our sample was based on spectroscopic classification, comparing our SN spectra with those of known SNe Ic-BL. We inspected the spectra of all SNe classified as possible SNe Ic and SNe Ic-BL in the PTF archive, and defined our final sample of SNe Ic-BL by cross-correlating our SN spectra with those included as templates in the Supernova Identification (SNID; Blondin \& Tonry 2007) program. We also used as templates those by Liu \& Modjaz (2014), specific for SE SNe. In Fig. 1 we present for each SN an example of SNID matches to SN Ic-BL spectral templates. As in Modjaz et al. (2016), PTF10bzf (SN 2010ah), PTF10qts, and PTF10vgv are included in our SN Ic-BL sample. If we use the method proposed by Prentice \& Mazzali (2017) to classify helium-poor SNe based on the number of absorption features in the spectra at and before the light-curve peak, our spectra show between two, three, and four features in most of the cases. This corresponds to SNe Ic with larger line blending caused by their fast velocities. Spectroscopically normal SNe Ic typically show six or seven features. We report the Ic- $\langle N\rangle$ classification (Prentice \& Mazzali 2017) for each SN observed before peak in Table A.1, after visual inspection of all the spectra. When only past-peak spectra are available, upper limits for $\langle N\rangle$ are provided.

Given their spectra, we include iPTF15eov and iPTF16asu in our sample, even though these SNe are somewhat peculiar objects: the former is a slow riser, slow decliner, superluminous $\mathrm{SN}$, whereas the latter is a very luminous fast-rising event. We 


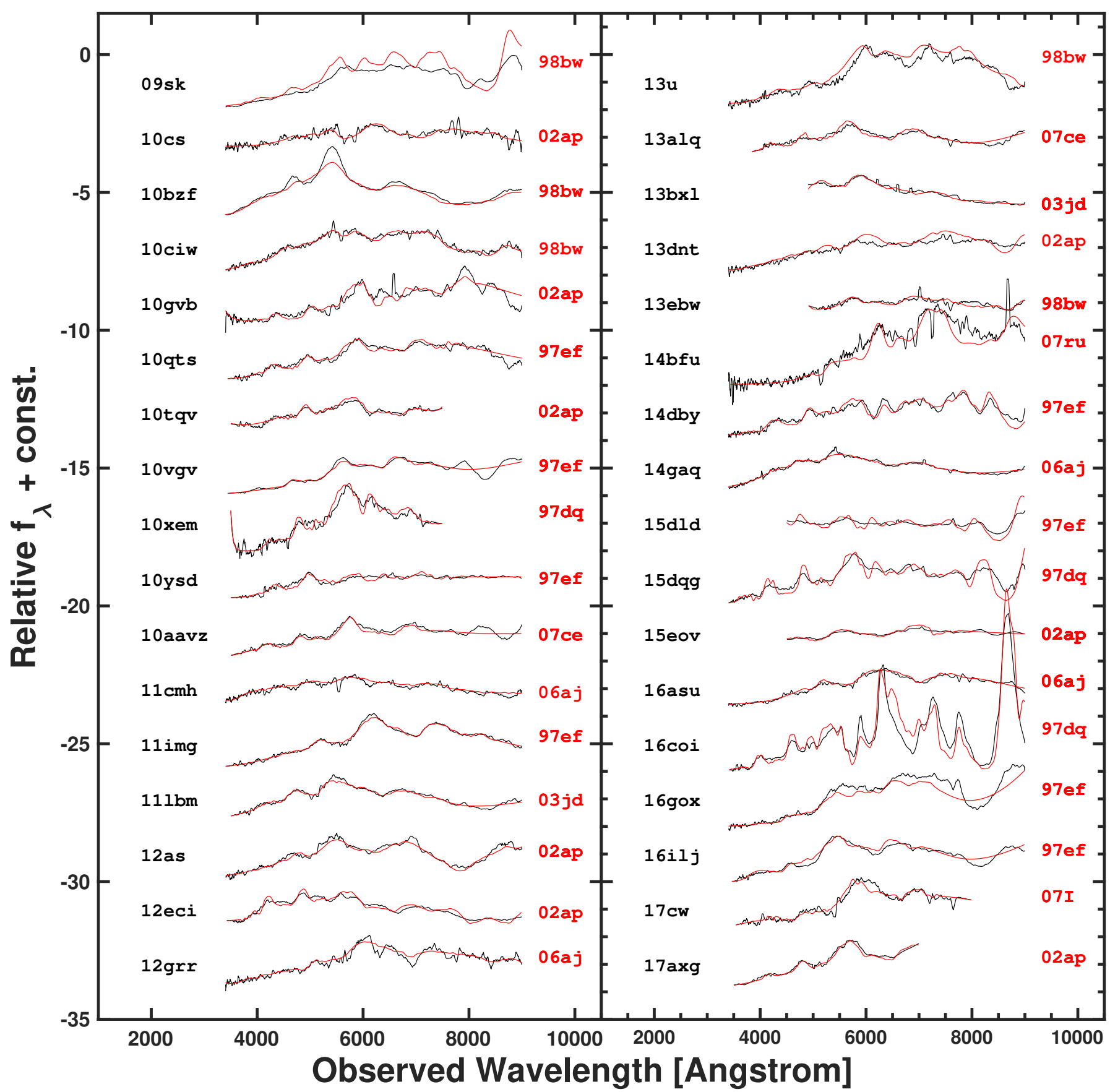

Fig. 1. SNID fits to the spectra of our 34 SNe Ic-BL. Next to each observed spectrum (in black) we report the SN name. The SN Ic-BL template spectrum that fits best is presented in red and the corresponding SN name is reported in red next to each spectrum. We selected a single spectrum for each SN, in order to show that they belong to the SN Ic-BL class.

describe their peculiarities with respect to the average properties of our sample throughout the paper, and discuss their underlying powering mechanism in Sect. 8.2. We also notice that if we use the classification code presented by Quimby et al. (2018), the spectra of iPTF15eov are better matched by SLSN-I than SN Ic spectra, whereas iPTF16asu is better matched by SN Ic spectra.

We measured the redshift of our SNe from their host galaxy spectral lines, often detected in the SN spectra or in archival host galaxy spectra. When the galaxy lines were not detected, as in the case of PTF12grr, the best SNID fit was used to estimate the redshift. Our SNe have the redshift distribution shown in Fig. 2. The average redshift is $0.090 \pm 0.069$; the most dis- tant object are located at $z=0.384$ and the most nearby at $z=0.0036$. The $\mathrm{SN}$ redshifts and host galaxy names are reported in Table 1. We did not include in our sample the transient at $z=$ 1.9733 named iPTF14yb, which was presented by Cenko et al. (2015) and associated with GRB 140226A; given its large distance, for this transient we obviously have only afterglow data available.

We computed the luminosity distance of each SN based on its redshift and assuming cosmological parameters from the fiveyear Wilkinson Microwave Anisotropy Probe (WMAP) observations $\left(H_{0}=70.5 \mathrm{~km} \mathrm{~s}^{-1} \mathrm{Mpc}^{-1}, \Omega_{\mathrm{m}}=0.27, \Omega_{\Lambda}=0.73\right.$; Komatsu et al. 2009), including corrections for peculiar motions (Virgo+GA+Shapley, as in Mould et al. 2000). The Milky Way 


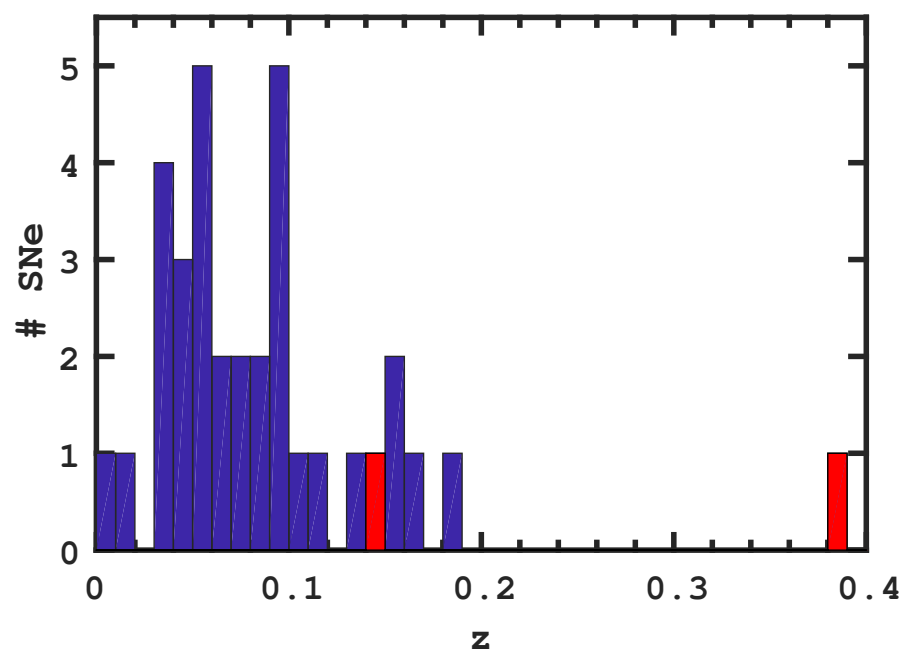

Fig. 2. Redshift distribution for our sample of SNe Ic-BL. All events are at $z<0.2$, except iPTF14bfu at $z=0.384$, which was targeted by iPTF in order to look for the optical counterpart of GRB 140606B (Cano et al. 2015). Also, iPTF13bxl $(z=0.145)$ was discovered while trying to find the optical counterpart of GRB 130702A (Singer et al. 2013). The two GRB SNe are marked in red.

(MW) color excess was obtained from Schlafly \& Finkbeiner (2011) via the NASA/IPAC Extragalactic Database (NED). Both the distances and $E(B-V)^{\mathrm{MW}}$ are listed in Table 1 .

We report $g$-band absolute magnitudes $\left(M_{g}^{\text {gal }}\right)$ for each host galaxy, as obtained through the apparent magnitude listed in the Sloan Digital Sky Survey (SDSS) catalog 2 or, when not observed by SDSS, in the Pan-STARRS catalog ${ }^{3}$. These galaxy magnitudes were K-corrected using their $g-r$ colors ${ }^{4}$ (Chilingarian et al. 2010; Chilingarian \& Zolotukhin 2012). For the host galaxies of iPTF14bfu and iPTF13bxl, we obtained the $g$ and $r$ magnitudes from Cano et al. (2015) and D'Elia et al. (2015), respectively. The absolute galaxy magnitudes span a relatively large range, from $M_{g}^{\mathrm{gal}}=-14.6 \mathrm{mag}$ to $M_{g}^{\mathrm{gal}}=$ $-21.2 \mathrm{mag}$, with average $\left\langle M_{g}^{\mathrm{gal}}\right\rangle=-18.3 \pm 0.3 \mathrm{mag}$.

From the post-peak $g-r$ colors of our SNe, we inferred the host galaxy extinction using a method similar to that illustrated by Stritzinger et al. (2018). The details of the method are given in Sect. 4.3. The values of $E(B-V)^{\text {host }}$ are listed in Table 1.

We also attempted to measure the equivalent width (EW) of the narrow $\mathrm{NaI} \mathrm{D}$ line in the $\mathrm{SN}$ spectra, and derive the host galaxy color excess using the formula provided by Taubenberger et al. (2006). These $E(B-V)^{\text {host }}$ values from Na I D are also reported in Table 1 . However, we detected the Na I D narrow absorption lines in just five $\mathrm{SNe}$, likely because our spectra of most $\mathrm{SNe}$, which are rather distant, do not have the necessary resolution and signal-to-noise ratio. Since we could not measure the $\mathrm{Na}$ I $\mathrm{D} \mathrm{EW}$ for most of our $\mathrm{SNe}$, and given that it is known that the Na I D EW is a poor proxy for host extinction (see, e.g., Phillips et al. 2013), we resorted to use the SN colors to estimate the host extinction.

Light curves of each $\mathrm{SN}$ are shown in Fig. 3. Our sample was mainly observed in the $r$ and $g$ bands (Sect. 3) and some photometric data were also obtained in the $B, i$, and $z$ bands. The light curves are presented, in the observer frame, in apparent magnitudes as a function of days since PTF/iPTF discovery. The

\footnotetext{
http://skyserver.sdss3.org

http://archive.stsci.edu/panstarrs/search.php

4 http://kcor.sai.msu.ru/about/
}

different bands are shifted by different amounts for clarity, and these shifts are indicated in the last subpanel of Fig. 3. Of our 34 events, we have $28 \mathrm{SNe}$ observed before $r$-band maximum brightness (or $g$-band maximum in the case of iPTF16asu and iPTF17axg). In the following sections, we only analyze those 28 $\mathrm{SNe}$ with at least one band observed before maximum brightness.

Our SNe were observed in the $r$ band with a median cadence of $3 \mathrm{~d}$ and for a median duration of $61 \mathrm{~d}$ and with a minimum duration of $6 \mathrm{~d}$ (PTF10cs) and a maximum duration of $150 \mathrm{~d}$ (PTF10aavz). We thus observed our SNe Ic-BL mainly during their photospheric phases.

For each SN, the collaboration also obtained spectra, mainly thanks to Caltech astronomers using the Keck (1 and 2) and Palomar (P200) telescopes. The epochs of the spectra are indicated by vertical black segments in Fig. 3 . The first spectral observation occurred before $r$-band maximum for 20 out of the $34 \mathrm{SNe}$. Many of our $\mathrm{SNe}$ were, in fact, imaged right after explosion, as demonstrated by the tight pre-explosion limits discussed in Sect. 6.1.

In summary, this PTF+iPTF photometric dataset of $\mathrm{SNe}$ Ic-BL is characterized by its untargeted nature, its large size, early coverage, high cadence, multiband coverage, and the availability of pre-explosion images. In addition, we present accompanying low-resolution spectroscopy to aid the analysis.

Several of the SNe in our sample have already been presented elsewhere. We report a list of references in the last column of Table 1. Nine of our SNe were described in single-object papers (marked in boldface in Table 1). All data from PTF+iPTF were already published for these $\mathrm{SNe}$ in the abovementioned papers, except in the cases of iPTF14bfu, iPTF15dld, and iPTF16coi, for which we provide additional data. The other previously reported $\mathrm{SNe}$ in our sample were included in sample papers. In particular, Corsi et al. (2016) presented radio observations and also the PTF/iPTF $r$-band light curves and classification spectra of 15 $\mathrm{SNe}$. For all these 21 previously studied $\mathrm{SNe}-$ as well as for the $13 \mathrm{SNe}$ presented for the first time in this paper - we provide a new analysis in the context of our large, untargeted, and homogeneous SN Ic-BL sample.

\section{Photometry acquisition and reduction}

Photometric observations were performed with the 48 inch Samuel Oschin Telescope at Palomar Observatory (P48), equipped with the $92 \mathrm{Mpx}$ mosaic camera $\mathrm{CFH} 12 \mathrm{~K}$ (Rahmer et al. 2008), a Mould $r$-band filter (Ofek et al. 2012), and an SDSS-like $g$-band filter (and in one case also an $i$-band filter). The P48 observations allowed the discovery of our SNe. Twenty-four of our SNe were also observed with the automated Palomar 60 inch telescope (P60; Cenko et al. 2006) in the Bgriz bands.

Point spread function (PSF) photometry was obtained on template-subtracted P48 and P60 images using the Palomar Transient Factory Image Differencing and Extraction (PTFIDE) pipeline (Masci et al. 2017) for P48 and the FPipe pipeline presented by Fremling et al. (2016) for P60. The P48 templates were obtained from the stack of pre-explosion images. As P60 templates, we used SDSS images in the corresponding filters (and Pan-STARRS images in the cases of PTF10cs, iPTF14bfu, and iPTF15eov, which did not have SDSS coverage). The photometry was calibrated against SDSS (or Pan-STARRS) stars (Ahn et al. 2014) in the SN field and is presented in AB magnitudes. When P48 and P60 photometry in $g$ and $r$ did not match perfectly owing to slightly different filters, we scaled the P48 


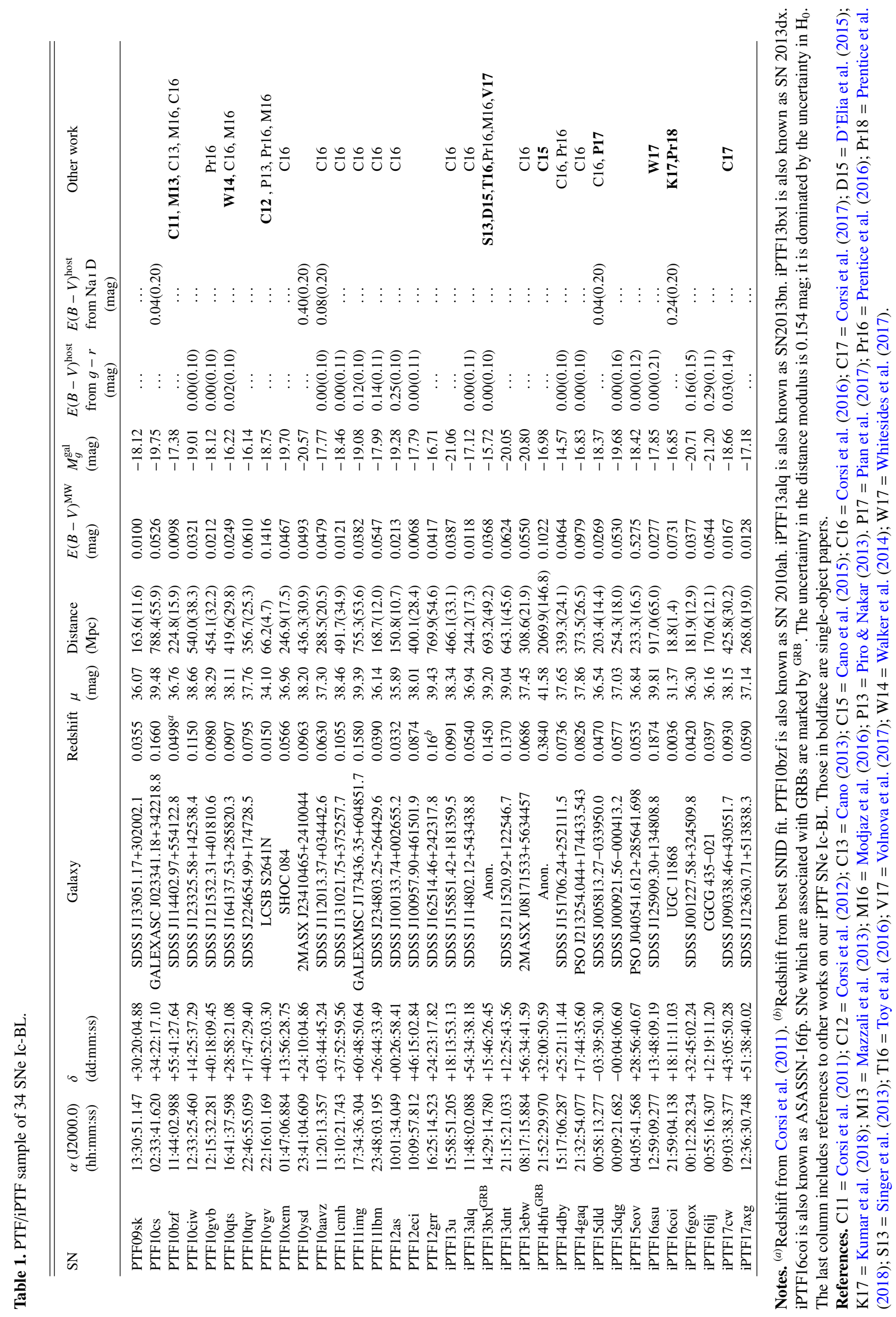




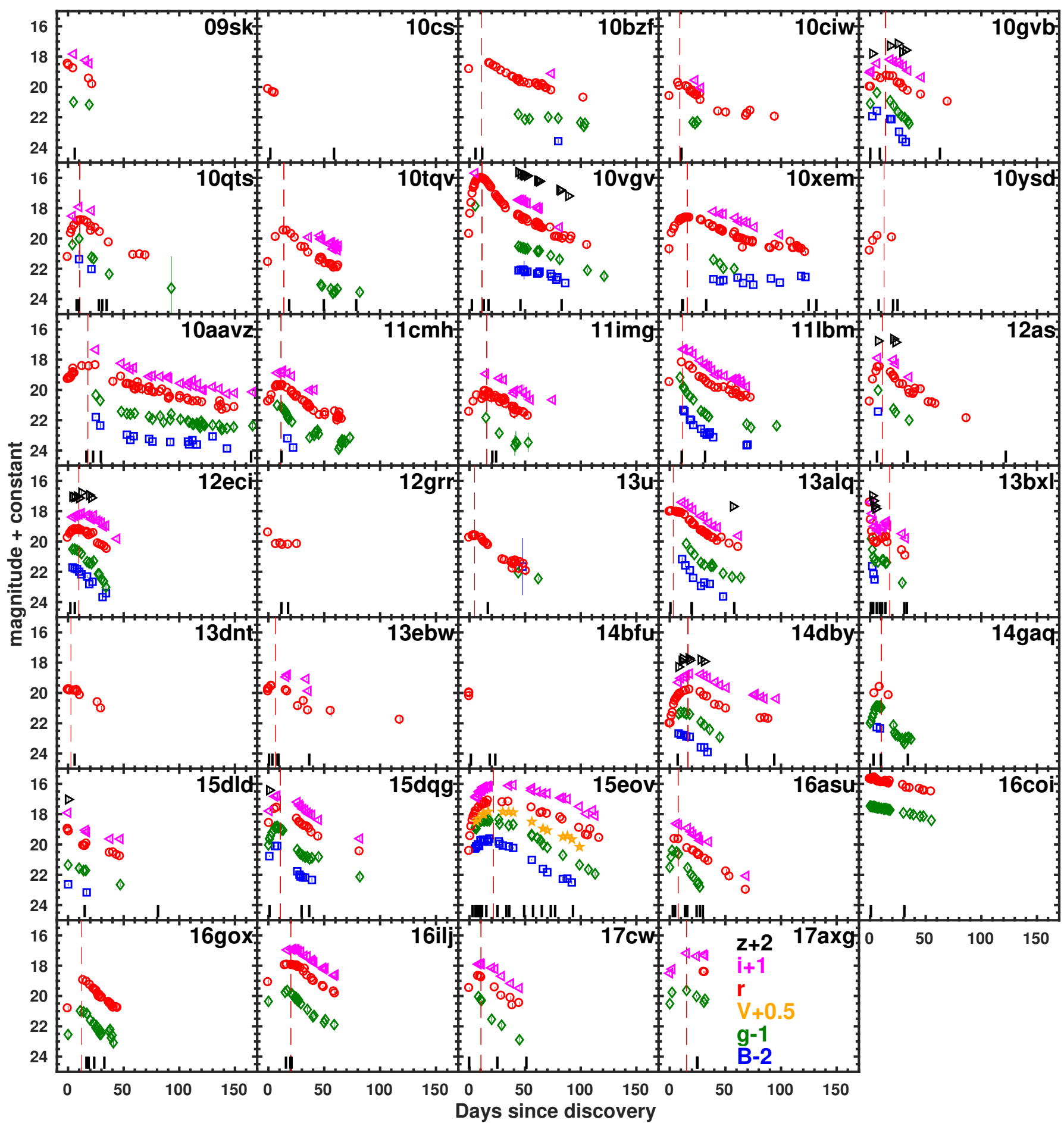

Fig. 3. $B$ (blue squares), $g$ (green diamonds), $r$ (red circles), $i$ (magenta triangles), and $z$ (black triangles) photometry for our sample of SNe Ic-BL. In the case of iPTF15eov we also have a $V$-band light curve (yellow stars). The light curves as a function of discovery epoch have been shifted for clarity (see the legend in the last subpanel). The epochs of the observed $r$-band maxima are marked by vertical red dashed lines. The spectral epochs are shown by black segments at the bottom of each subpanel.

photometry to match the $\mathrm{P} 60$ data points by adding to the $\mathrm{P} 48$ light curve the mean magnitude difference between $\mathrm{P} 48$ and P60 in the time range where P48 and P60 data overlap. For example, P48 mounts a Mould $r$ filter, while P60 is equipped with an $r$ filter closer to the standard SDSS. In the case of iPTF15eov, BgVri data were also obtained by the Las Cumbres Observatory (LCO) and reduced using the LCO pipeline lcogtsnpipe (Valenti et al. 2016), a PyRAF-based photomet- ric reduction pipeline. We performed point spread function photometry. Reference images were obtained with SBIG and Sinistro after the $\mathrm{SN}$ faded and image subtraction was performed using PyZOGY (Guevel \& Hosseinzadeh 2017), an implementation in Python of the subtraction algorithm described by Zackay et al. (2016). The $B V$ data and gri data were calibrated to Vega magnitudes and AB magnitudes, respectively. iPTF16asu was also observed with Telescopio Nazionale Galileo (TNG) 


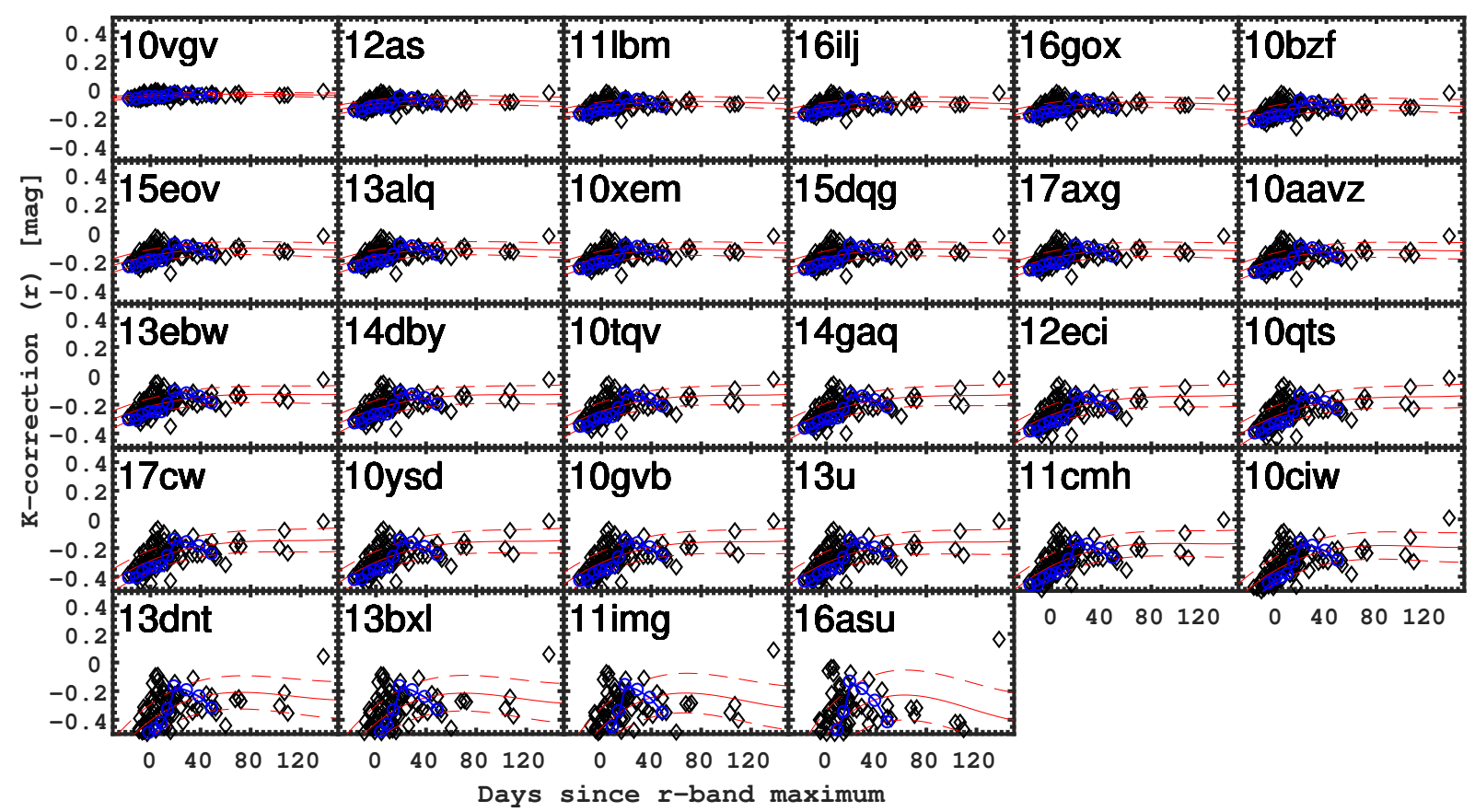

Fig. 4. K-corrections in the $r$ band for our SN sample. For each SN redshift we determined the $K_{\text {corr }}^{r}(z, t)=r_{\text {observed }}-r_{\mathrm{K} \text {-corrected }}$ from all the SN spectra in our sample, and we fit them with a second-order polynomial, shown as a solid red line. The $1 \sigma$ uncertainties are shown as red dashed lines. The SNe are ordered based on redshift, with the nearest ones on top. For the most distant events, K-corrections in $r$ have values up to 0.4 mag around peak. For comparison, in blue we show the K-corrections from the SN Ic-BL spectral templates available at Peter Nugent's Spectral Templates webpage. The results are similar and compatible within the uncertainties.

in gri at late epochs (see Whitesides et al. 2017). The final SN magnitudes in each band will be made available on WISeREP (Yaron \& Gal-Yam 2012).

\section{Supernova light curves and colors}

Figure 3 provides all of the photometric observations obtained in the optical bands for our SN sample. We now proceed to the analysis of the $28 \mathrm{SNe}$ observed before peak brightness. The first step is to correct the observed light curves for time dilation and $\mathrm{K}$-corrections, given their small but not negligible effects on the light-curve shape.

\subsection{K-corrections}

We first determined the observed peak epoch in the $r$ band $\left(t_{r}^{\max }\right)$, given that most of our $\mathrm{SNe}$ were observed at peak in that filter. This was done by fitting a polynomial to the $r$-band light curves. The peak epochs are indicated with a dashed red line in Fig. 3. In the cases of iPTF16asu and iPTF17axg, the $r$-band peak epoch was estimated from the $g$-band peak epoch using the formula presented by Taddia et al. (2015).

Armed with measured $t_{r}^{\max }$, we determined a phase $t$ for each spectrum of the SNe in our sample. All phases are given in the rest frame, after correcting for time dilation - that is, correcting the observed phase by a factor $(1+z)$. A log of the spectra is provided in Table A.1, and all of the spectra are presented and analyzed in Sect. 5. With these spectra, we computed average $\mathrm{K}$-corrections for the Bgriz bands as functions of redshift and time since $t_{r}^{\max }$.

Ideally, K-corrections for each individual SN should be calculated using a comprehensive spectral sequence for the given object. Unfortunately, we have very few spectra per SN, but instead have a large sample of SE SN spectra in general. We therefore proceeded in the following way. For each given SN observed in a filter centered on $\lambda$, (i) we considered the redshift $z$ of the SN, and shifted all the rest-frame spectra in our entire sample to that redshift using $f_{\lambda}^{\text {rest }}=f_{\lambda}^{\text {observed }}(1+z)$ and $\lambda_{\text {rest }}=\lambda_{\text {observed }} /(1+z)$. (ii) Next, we computed for each spectrum the synthetic magnitude in the given filter $\lambda$ at that redshift $\left(m_{z, t}\right)$ and in the rest frame $\left(m_{z=0, t}\right)$. From each spectrum, we first removed the host galaxy emission lines and we discarded those spectra that were strongly contaminated by the host continuum. We also first corrected all of the spectra for MW extinction. (iii) Then, we defined the K-correction from each spectrum in the sample as $K_{\text {corr }}^{\lambda}(z, t)=m_{z, t}-m_{z=0, t}$, with $t$ being the phase of the spectrum. (iv) We plotted all of the obtained $K_{\text {corr }}^{\lambda}(z, t)$ as a function of phase and fit them with a secondorder polynomial. The fits for the $r$ band are shown in Fig. 4. Kcorrections are larger at early epochs. (v) Once we obtained the $\mathrm{K}$-correction polynomials in all of the bands and for each SN, we K-corrected all of the Bgriz light curves by the values obtained with the interpolation of the polynomials at the epochs of the different light-curve observations. The average $1 \sigma$ uncertainty in the K-corrections between -20 and +40 days for the $r$ band is 0.07 mag.

In the following analysis, we always refer to our K-corrected and time-dilation-corrected light curves. K-corrections can be as large as $\sim 0.5 \mathrm{mag}$ at peak brightness in the $r$ band (see Fig. 4), but for most of the events these corrections are smaller than $0.3 \mathrm{mag}$ at the same phase and subsequently even lower. For comparison, we also obtained the $r$-band K-corrections from Peter Nugent's spectral template of SNe Ic-BL ${ }^{5}$, and we plot these as well in Fig. 4. The results are compatible within the uncertainties.

\footnotetext{
5 Available at this webpage: https://c3.lbl.gov/nugent/ nugent_templates.html.
} 


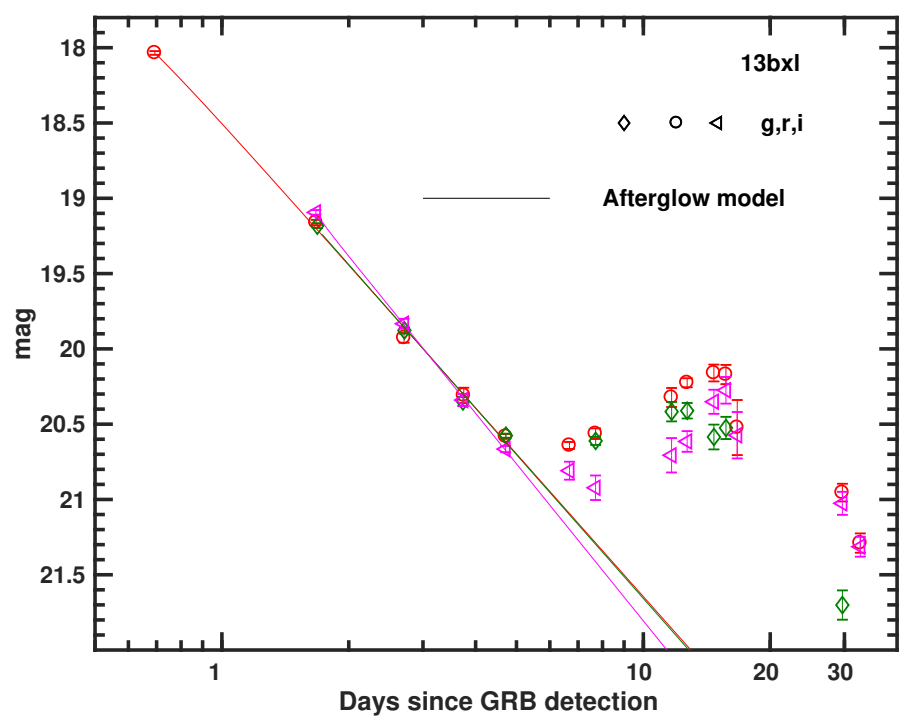

Fig. 5. Fit of the afterglow emission from the gri light curves of iPTF13bxl.

\subsection{Light-curve shape}

Armed with the K-corrected light curves, we proceeded to fit the $r$-band light curves with the function provided by Contardo et al. (2000) to characterize their shape. This function includes an exponential rise, a Gaussian peak, and a linear late decline, as also discussed in Taddia et al. (2018a). Only the $27 \mathrm{SNe}$ observed both before and after $r$-band peak were included; iPTF17axg was observed before peak but only in $g$ band. From the functional fit, it is easy to derive the peak epoch and peak magnitude, the decline parameter $\Delta m_{15}$, and the rise parameter $\Delta m_{-10}$, as well as the late linear-decline slope.

The results of our best fits to the $r$-band light curves are shown in Fig. 6. In the top panel, each $\mathrm{SN}$ is reported in individual subpanels, whereas in the bottom panel we overplot all of our $\mathrm{SNe}$ to better show the general shape of their light curves. The latter panel highlights the large variety of rise and decline rates; broad and narrow light curves are part of our sample. iPTF15eov emerges as the broadest event, clearly separated from the rest of the SNe.

We estimate uncertainties in each of the light-curve-fit parameters by Monte Carlo simulating 1000 light curves according to their photometric uncertainties and refitting them with the same function. The standard deviations of the best-fit parameters were taken as the uncertainty on each parameter. The parameters and their estimated uncertainties are reported in Table 2.

Figure 7 suggests a correlation between $\Delta m_{15}$ and $\Delta m_{-10}$ where fast-rising objects are also fast decliners. The correlation is characterized by a $p$-value of 0.006 if we perform a Spearman correlation test. We also tested if $\Delta m_{15}$ correlates with the latetime linear slope, but given the small number of SNe observed at late epochs (seven $\mathrm{SNe}$ with a measured late-time slope and $\Delta m_{15}$ ) we could not statistically confirm the correlation found by Taddia et al. (2018a). Our test gives a $p$-value of 0.55 .

We note that in the case of iPTF13bxl, the light curves are characterized by a strong GRB afterglow in the optical that precedes the SN emission (see Fig. 5). This characteristic of the GRB SN iPTF13bxl was studied in detail by Singer et al. (2013), D'Elia et al. (2015), and Toy et al. (2016), where the association of iPTF13bxl to GRB 130702A was investigated. Therefore, before performing the light-curve fit with the Contardo et al.
(2000) function, we fit and removed the afterglow emission using the method presented by Cano et al. (2011, see their Eq. (2)). The afterglow fits to the epochs $<5 \mathrm{~d}$ since the GRB are indicated by solid lines in Fig. 5 for the gri light curves.

\subsection{Observed g-r colors and host extinction}

We proceeded with the colors of our SNe, in particular $g-r$. We made use of the $g-r$ colors to estimate the host extinction, following the approach of Stritzinger et al. (2018).

For each SN with both $g$ and $r$ observations both before and after $r$-band maximum, we interpolated the $r$ observations to the $g$-band epochs, and plotted the obtained $g-r$, after having corrected both bands for MW extinction. This was done adopting the MW $E(B-V)$ given in Table 1 , assuming $R_{V}=3.1$ and the Fitzpatrick (1999) reddening law.

The $g-r$ colors in the time interval between 0 and $20 \mathrm{~d}$ after $r$-band maximum are shown in Fig. 8. We also illustrate a $g-r$ color template between 0 and $20 \mathrm{~d}$ after $r$ maximum, which we obtained from the extinction-corrected $g-r$ colors presented for six relatively nearby $(z<0.05)$ SNe Ic-BL (Prentice et al. 2016, see their Fig. 10).

The $g-r$ colors of the SNe presented by Prentice et al. (2016) were corrected for the host extinction mainly based on the measurement of narrow Na I D absorption in the SN spectra. We took the average and standard deviation of these colors at each epoch between 0 and $20 \mathrm{~d}$ as the intrinsic color template. The standard deviation of our $g-r$ template is $\sim 0.1 \mathrm{mag}$.

An alternative approach to obtaining the intrinsic color for SNe Ic-BL would be to identify apparently unreddened events in our sample and choose their colors as intrinsic. We could identify, among the objects with the bluest colors that also had no detected $\mathrm{Na}$ I $\mathrm{D}$ at the host galaxy rest wavelength, those with absolute magnitude (as computed assuming no host extinction) not too far from the middle of the absolute-magnitude distribution in our sample. Similar assumptions were made in the selection of the intrinsic colors of SE SNe by Stritzinger et al. (2018), but they only had two SNe Ic-BL. For our sample, PTF12eci would match these criteria, and we could therefore assume its $g-r$ color as the intrinsic $g-r$ color for SNe Ic-BL. In fact, the $g-r$ color of PTF12eci (see Fig. 8) turns out to be consistent, within the errors, with the $g-r$ template that we obtained from the dereddened $g-r$ color of the best-observed SNe Ic-BL from the literature (from Prentice \& Mazzali 2017). Therefore, we proceed assuming the $g-r$ template as the intrinsic $g-r$ color.

We fit the $g-r$ colors of all the SNe with low-order polynomials, shown as solid, colored lines in Fig. 8. We then computed the average $E(g-r)$ for each of our SNe in the range between 0 and $20 \mathrm{~d}$ since peak brightness by computing the average difference between the fit to the observed $g-r$ color and the assumed intrinsic $g-r$ color. Next we converted $E(g-r)$ into $E(B-V)$ host assuming $R_{V}^{\text {host }}=3$.1. Using the canonical $R_{V}^{\text {host }}=3.1$ allows for easy comparisons with other work in the literature. We also notice that for the only SN Ic-BL (SN 2009bb) for which Stritzinger et al. (2018) could estimate the $R_{V}^{\text {host }}$, the value was consistent with the usual 3.1 (it was $3.3_{-0.3}^{+0.4}$ ).

The computed $E(B-V)^{\text {host }}$ are reported in Table 1 and shown in Fig. 8. The uncertainties in the host galaxy extinction include the uncertainty of the $g-r$ template, and they also account for the standard deviation of the difference between each epoch of the intrinsic and measured $g-r$ color. For the SNe without observed $g-r$ color in the range between 0 to $20 \mathrm{~d}$ past 

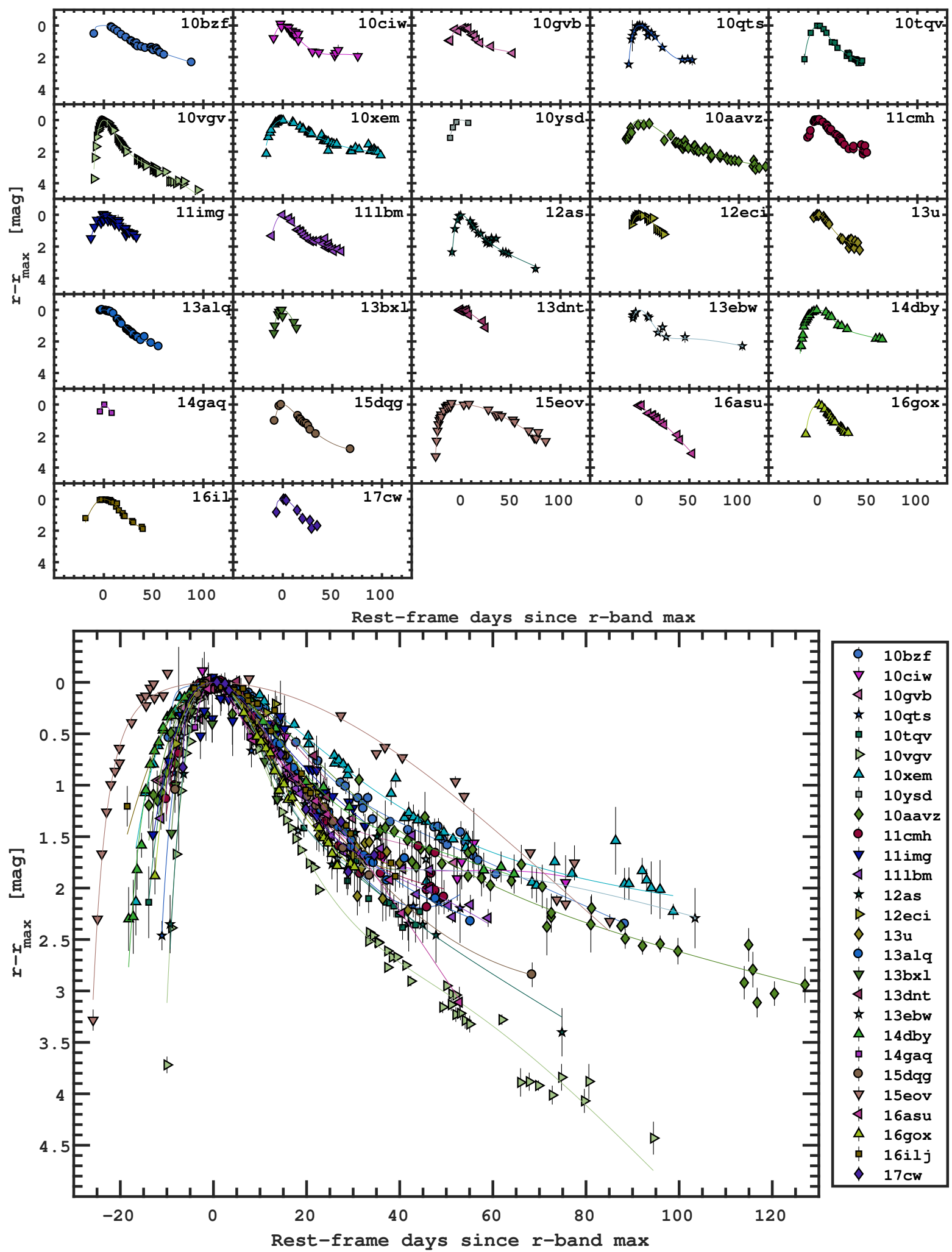

Fig. 6. Top panel: rest-frame $r$-band light curves fit by the Contardo et al. (2000) equation. The best fits are shown as solid lines, and the light curves have been normalized to peak brightness. Bottom panel: same as in the top panel, but now all SNe were overplotted to highlight the variety of rise and decay timescales.

maximum, we adopted the host extinction obtained from the measured equivalent width of $\mathrm{Na}$ I $\mathrm{D}$, reported in Table 1 , and then used Eq. (1) of Taubenberger et al. (2006). For those cases, we adopted an uncertainty of $E(B-V)=0.2 \mathrm{mag}$. If no Na I D was detected and no $g-r$ color excess could be computed, we assumed the host extinction to be negligible. This was done for $11 \mathrm{SNe}$, which are those in Table 1 without indications for $E(B-V)^{\text {host }}$. We notice that the colors of iPTF15eov are particularly blue, as are those of iPTF16asu. These two SNe are also peculiar in other respects within our sample - iPTF15eov 
Table 2. $r$-band light-curve properties and explosion epochs.

\begin{tabular}{|c|c|c|c|c|c|c|c|}
\hline SN & $\begin{array}{c}t\left(r_{\max }\right) \\
(\mathrm{JD})\end{array}$ & $\begin{array}{c}r_{\max } \\
(\mathrm{mag})\end{array}$ & $\begin{array}{l}\Delta m_{15} \\
(\mathrm{mag})\end{array}$ & $\begin{array}{l}\Delta m_{-10} \\
(\mathrm{mag})\end{array}$ & $\begin{array}{c}\text { Linear slope } \\
\left(\mathrm{mag} \mathrm{d}^{-1}\right)\end{array}$ & $\begin{array}{l}M_{r}^{\max } \\
(\mathrm{mag}) \\
\end{array}$ & $\begin{array}{c}t_{\text {explo }}{ }^{a} \\
(\mathrm{JD})\end{array}$ \\
\hline PTF10bzf & $2455261.03(1.91)$ & $18.48(0.06)$ & $0.43(0.05)$ & $\ldots$ & $0.019(0.001)$ & $-18.30(0.75)$ & 2455246.94 \\
\hline PTF10ciw & $2455262.38(0.26)$ & $20.22(0.11)$ & $0.77(0.05)$ & $\ldots$ & $0.060(0.005)$ & $-18.53(0.41)$ & 2455242.69 \\
\hline PTF10gvb & $2455335.97(0.16)$ & $19.42(0.06)$ & $0.82(0.01)$ & $0.73(0.01)$ & $\ldots$ & $-18.92(0.41)$ & 2455316.10 \\
\hline PTF10qts & $2455425.84(0.04)$ & $19.10(0.05)$ & $0.74(0.01)$ & $1.67(0.09)$ & $\ldots$ & $-19.13(0.39)$ & 2455412.10 \\
\hline PTF10tqv & $2455437.88(1.12)$ & $19.73(0.15)$ & $1.09(0.08)$ & $0.86(0.16)$ & $\ldots$ & $-18.19(0.76)$ & 2455422.67 \\
\hline PTF10vgv & $2455463.78(0.02)$ & $16.06(0.01)$ & $1.24(0.01)$ & & $0.067(0.013)$ & $-18.41(0.74)$ & 2455453.47 \\
\hline PTF10xem & $2455486.44(0.16)$ & $18.80(0.04)$ & $0.35(0.01)$ & $0.40(0.01)$ & $0.032(0.007)$ & $-18.28(0.75)$ & 2455461.65 \\
\hline PTF10ysd & $2455491.16(0.38)$ & $20.05(0.05)$ & & $0.75(0.16)$ & & $-19.31(0.75)$ & 2455465.00 \\
\hline PTF10aavz & $2455529.12(0.54)$ & $18.34(0.04)$ & $0.48(0.05)$ & $0.56(0.15)$ & $0.016(0.001)$ & $-19.08(0.39)$ & 2455507.98 \\
\hline PTF11 cmh & $2455682.98(0.07)$ & 20.03(0.03) & $0.80(0.09)$ & $1.24(0.21)$ & $\ldots$ & $-18.46(0.42)$ & 2455669.28 \\
\hline PTF11img & $2455767.27(1.07)$ & $20.53(0.04)$ & $0.64(0.16)$ & $0.85(0.32)$ & $\ldots$ & $-19.28(0.39)$ & 2455748.34 \\
\hline PTF11lbm & $2455805.47(0.10)$ & $18.32(0.01)$ & $0.86(0.01)$ & $1.00(0.10)$ & $\ldots$ & $-18.32(0.41)$ & 2455793.76 \\
\hline PTF12as & $2455933.55(0.50)$ & $18.45(0.06)$ & $1.17(0.09)$ & $2.07(0.30)$ & $0.045(0.005)$ & $-18.14(0.39)$ & 2455921.01 \\
\hline PTF12eci & $2456067.34(0.13)$ & $19.48(0.02)$ & $0.63(0.05)$ & $\ldots$ & $\ldots$ & $-18.55(0.43)$ & 2456052.37 \\
\hline iPTF13u & $2456330.19(0.22)$ & $19.93(0.04)$ & $0.95(0.06)$ & $\ldots$ & $\ldots$ & $-18.51(0.75)$ & 2456318.90 \\
\hline iPTF13alq & $2456398.28(0.10)$ & $18.19(0.02)$ & $0.63(0.01)$ & $\ldots$ & $\ldots$ & $-18.77(0.42)$ & 2456386.46 \\
\hline iPTF13bxl & $2456492.01(0.60)$ & 20.36(0.09) & & $\ldots$ & $\ldots$ & $-18.94(0.40)$ & 2456475.00 \\
\hline iPTF13dnt & $2456566.01(0.63)$ & $20.21(0.06)$ & $0.49(0.04)$ & $\ldots$ & $\ldots$ & $-19.00(0.75)$ & 2456545.04 \\
\hline iPTF13ebw & $2456630.13(0.19)$ & $19.56(0.01)$ & $1.27(0.18)$ & $\ldots$ & $0.007(0.004)$ & $-18.03(0.74)$ & 2456612.21 \\
\hline iPTF14dby & $2456850.34(0.29)$ & $20.00(0.07)$ & $0.61(0.03)$ & $0.38(0.01)$ & $\ldots$ & $-17.77(0.40)$ & 2456828.08 \\
\hline iPTF14gaq & $2456930.24(0.22)$ & $19.88(0.03)$ & & $\ldots$ & $\ldots$ & $-18.23(0.40)$ & 2456920.21 \\
\hline iPTF15dqg & $2457342.21(0.20)$ & $17.75(0.01)$ & $0.75(0.03)$ & $\ldots$ & $\ldots$ & $-19.41(0.61)$ & 2457328.73 \\
\hline iPTF15eov & $2457386.96(0.45)$ & $17.38(0.02)$ & $0.11(0.01)$ & $0.07(0.01)$ & $\ldots$ & $-20.82(0.45)$ & 2457358.72 \\
\hline iPTF16asu & $2457525.65(0.09)$ & $20.09(0.10)$ & $0.83(0.05)$ & $\ldots$ & $\ldots$ & $-19.79(0.80)$ & 2457519.03 \\
\hline iPTF16gox & $2457647.26(4.92)$ & $19.06(0.15)$ & $0.24(0.30)$ & $\ldots$ & $\ldots$ & $-17.74(0.58)$ & 2457641.59 \\
\hline iPTF16ilj & $2457721.67(0.21)$ & $18.05(0.03)$ & $0.68(0.01)$ & $0.37(0.03)$ & $\ldots$ & $-19.00(0.42)$ & 2457701.25 \\
\hline iPTF17cw & $2457767.98(1.20)$ & $19.00(0.07)$ & $0.77(0.09)$ & $\ldots$ & $\ldots$ & $-19.26(0.54)$ & 2457752.40 \\
\hline
\end{tabular}

Notes. For the $27 \mathrm{SNe}$ Ic-BL observed before and after $r$ maximum brightness. ${ }^{(a)}$ Obtained by stretching the $R$-band light curve of SN $1998 \mathrm{bw}$ to the $r$-band light curves of each of our SNe. Typical uncertainty of $\pm 2 \mathrm{~d}$ (see text).

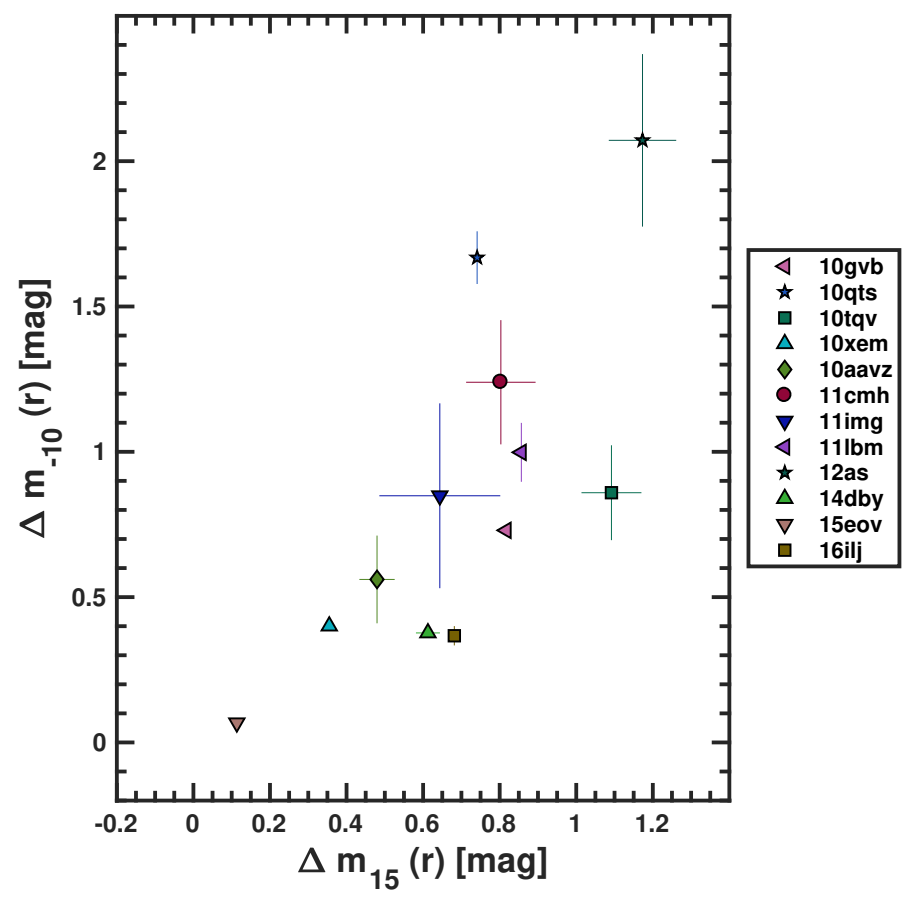

Fig. 7. $\Delta m_{-10}$ vs $\Delta m_{15}$ in $r$ for the sample of SNe Ic-BL. The correlation is significant ( $p$-value $=0.006$ using a Spearman test).

because of its unusually broad light curve and its extraordinary large peak brightness (see Sect. 4.4), and iPTF16asu for its nonstandard light curve with an unprecedented fast rise (as discussed in detail by Whitesides et al. 2017).

\subsection{Absolute magnitudes}

With the computed host galaxy extinctions, distances, and $\mathrm{K}$-corrected light curves, we proceeded to compute the absolutemagnitude light curves, which for the $r$ band are shown in the left-hand panel of Fig. 9. The uncertainty in the absolute magnitudes accounts for that in the host extinction and in the photometry. We further report the systematic uncertainty from the distance $( \pm 0.15 \mathrm{mag})$ in the upper-right corner of the left panel in Fig. 9. In the right-hand panel of Fig. 9, the distribution of the peak $r$-band absolute magnitudes is shown. Our sample ranges over $3 \mathrm{mag}$ in $M_{r}^{\max }$, between -17.7 and $-20.8 \mathrm{mag}$, with an average of $-18.7 \pm 0.7 \mathrm{mag}$. iPTF15eov is clearly brighter than all of the other $\mathrm{SNe}, \sim 1 \mathrm{mag}$ brighter than the second brightest (iPTF16asu). Its $r$-band peak is $4 \sigma$ brighter than the average of the other $\mathrm{SNe}$ in our sample.

If we exclude iPTF15eov and iPTF16asu from the average, as they might not belong to the sample of normal $\mathrm{SNe}$ Ic-BL (see Sect. 8.2), the average peak is $-18.6 \pm 0.5$ mag. If we compute the average peak absolute magnitude by weighting for the inverse effective search volume for each SN, we obtain $-18.4 \pm 0.4 \mathrm{mag}$. This value should be closer to the intrinsic absolute magnitude average since it takes into account the volume effect.

All of the $r$-band peak magnitudes are reported in Table 3, where the uncertainty accounts for the error on the host extinction and on the fit to the light curve. An additional systematic uncertainty of $0.15 \mathrm{mag}$ due to the systematic uncertainty in the distance should also be considered. The spread of absolute peak magnitudes is larger than the total uncertainty in the measured peaks, implying that there is an actual range of peak luminosity 


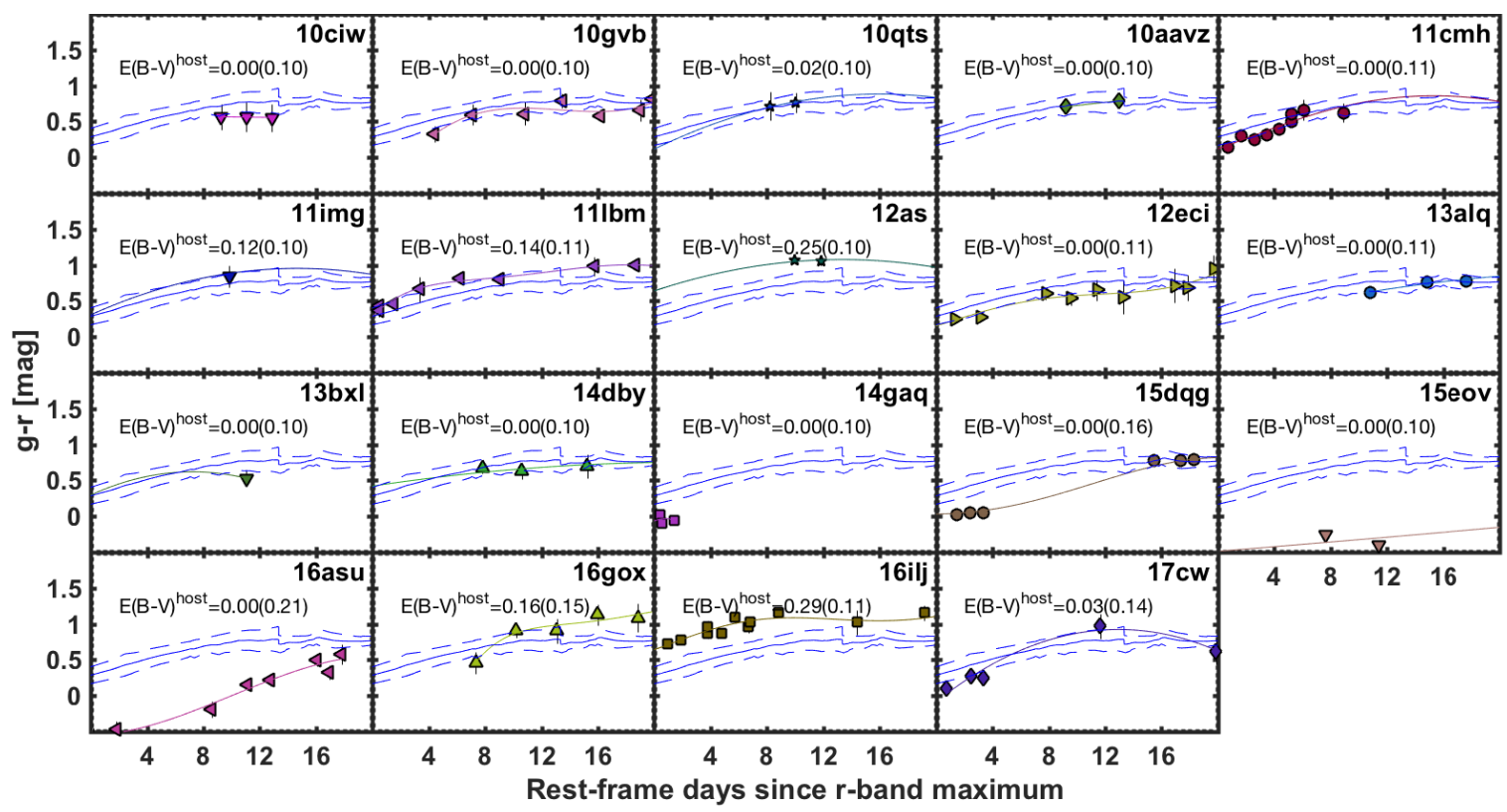

Fig. 8. $E(B-V)^{\text {host }}$ from the $g-r$ colors measured between 0 and $20 \mathrm{~d}$ after $r$-band peak brightness and corrected only for the MW extinction. The blue curves represent the intrinsic color and its uncertainty (obtained from the dereddened $g-r$ colors of six SNe Ic-BL from the literature (see Prentice et al. 2016, their Fig. 10). Each SN color is fit with a low-order polynomial (colored curves with color matching the symbol color) in order to compare it to the intrinsic color and compute the average $g-r$ color excess.
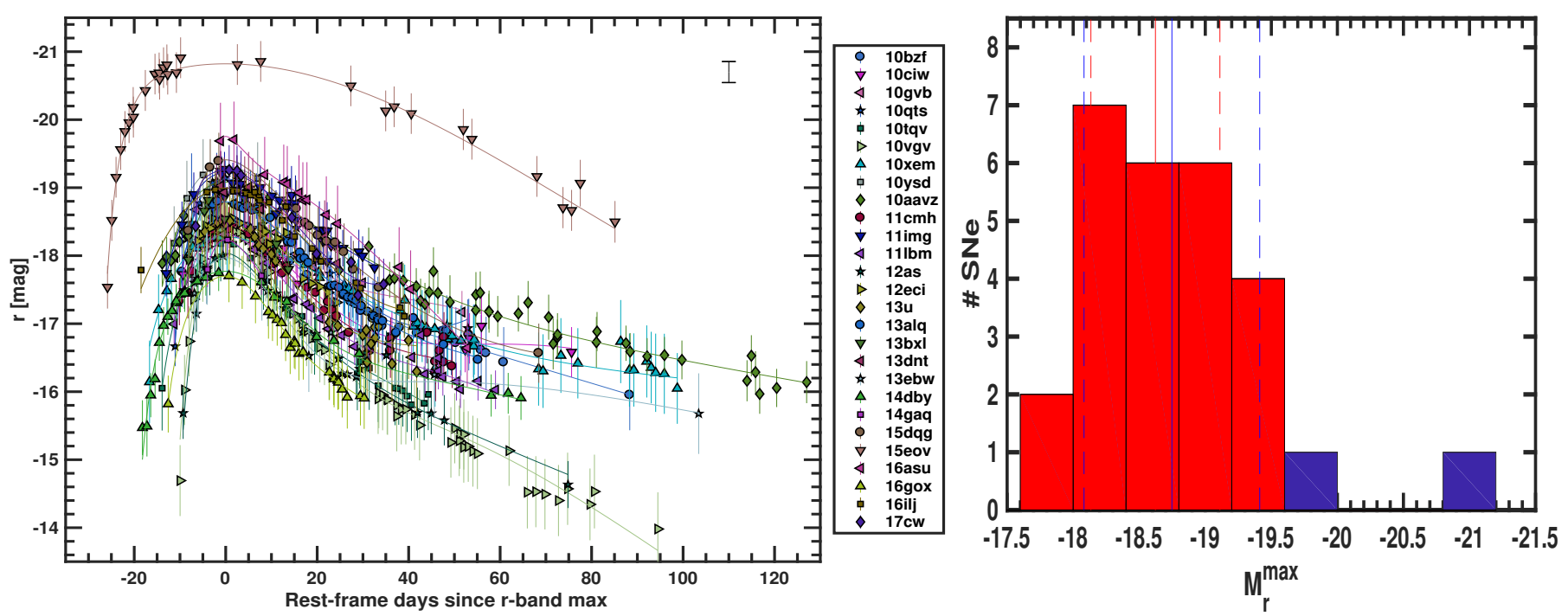

Fig. 9. Left panel: absolute $r$-band magnitude light curves, after (host $+\mathrm{MW}$ ) extinction correction and K-correction. The best fits are the same as those presented in Fig. 6. The systematic uncertainty in the distance is shown in the upper-right corner. Right panel: distribution of the absolute $r$ peak magnitudes for our sample. iPTF15eov and iPTF16asu are marked in blue. Blue vertical lines indicate the average and the standard deviation of the distribution when including all of the objects. Red vertical lines mark the average and the standard deviation of the distribution when excluding iPTF15eov and iPTF16asu.

in this family of transients. The distribution of the peak $r$-band absolute magnitudes is shown in the left panel of Fig. 9. In the other bands (Bgiz), we have fewer observations that include the peak of the light curves. For completeness, we plot their absolute magnitudes in Fig. 10 as a function of time since $r$-band maximum.

In Fig. 11 we plot the absolute $r$ magnitude peak $M_{r}^{\max }$ versus $\Delta m_{15}(r)$ to test if there is a Phillips relation (Phillips 1993) as in $\mathrm{SNe}$ Ia. Such a relation was also seen for GRB SNe by Cano (2014) and Li et al. (2014). The SNe Ic-BL in our sample do not follow such a relation (shown by a black line from Burns et al. 2011 in the figure). It is not always the case that the less luminous
SNe are also those that decline faster. This was also seen by Cano (2014) for SNe Ic-BL that are not associated with GRBs and for other SE SNe. In addition, we tested if there is any correlation between $M_{r}^{\max }$ and $\Delta m_{40}(r)$, but did not find any such correlation. Furthermore, the peak $r$-band magnitudes do not correlate with the slopes at late epochs.

\subsection{Colors}

We compute the intrinsic $g-r$ colors of our SNe including the corrections for host extinction. The intrinsic colors are shown in the upper right panel of Fig. 12. In the other panels, we 
A\&A 621, A71 (2019)

Table 3. Bolometric light curve properties and expansion velocities of the SNe listed in Table 2.

\begin{tabular}{|c|c|c|c|c|c|c|}
\hline $\mathrm{SN}$ & $\begin{array}{l}t(\text { bolo })_{\max } \\
(\mathrm{JD})\end{array}$ & $\begin{array}{l}M(\text { bolo })_{\max } \\
\quad(\mathrm{mag})\end{array}$ & $\begin{array}{l}\Delta m_{15}^{\text {bolo }} \\
(\mathrm{mag})\end{array}$ & $\begin{array}{c}\Delta m_{-10}^{\text {bolo }} \\
(\mathrm{mag})\end{array}$ & $\begin{array}{l}\text { Linear slope (bolo) } \\
\quad\left(\mathrm{mag} \mathrm{d}^{-1}\right)\end{array}$ & $\begin{array}{l}\text { Fe II velocity at } r \text {-band peak } \\
\qquad\left(\mathrm{km} \mathrm{s}^{-1}\right)\end{array}$ \\
\hline PTF10bzf & $2455260.57(2.96)$ & $-18.20(0.34)$ & $0.47(0.11)$ & $\ldots$ & $0.032(0.005)$ & 19237(1154) \\
\hline PTF10ciw & $2455258.68(0.14)$ & $-18.59(0.11)$ & $0.90(0.08)$ & $\ldots$ & $0.069(0.004)$ & $22036(8208)$ \\
\hline PTF10gvb & $2455332.79(0.79)$ & $-18.72(0.11)$ & $0.70(0.05)$ & $\ldots$ & $\ldots$ & 11952(1964) \\
\hline PTF10qts & $2455423.26(0.37)$ & $-19.00(0.10)$ & $0.78(0.09)$ & & $\ldots$ & $21830(923)$ \\
\hline PTF10tqv & $2455433.84(1.35)$ & $-18.49(0.26)$ & $1.30(0.05)$ & $1.75(0.01)$ & $\ldots$ & 18985(2298) \\
\hline PTF10vgv & $2455462.64(0.45)$ & $-18.23(0.06)$ & $1.19(0.10)$ & & $0.031(0.003)$ & $8401(2187)$ \\
\hline PTF10xem & $2455485.54(2.89)$ & $-18.10(0.14)$ & $0.33(0.02)$ & $0.45(0.03)$ & $\ldots$ & $11229(2268)$ \\
\hline PTF10ysd & $2455487.95(1.34)$ & $-19.25(0.10)$ & & & $\ldots$ & 10900(3336) \\
\hline PTF10aavz & $2455526.81(0.29)$ & $-18.72(0.28)$ & $0.41(0.04)$ & $0.50(0.09)$ & $0.013(0.001)$ & $12040(2129)$ \\
\hline PTF11cmh & $2455681.25(0.36)$ & $-18.34(0.06)$ & $0.84(0.19)$ & $\ldots$ & $\ldots$ & 15195(1852) \\
\hline PTF11img & $2455763.86(2.38)$ & $-18.98(0.21)$ & $0.49(0.19)$ & $1.02(0.09)$ & $\ldots$ & 19088(2049) \\
\hline PTF11lbm & $2455804.66(2.54)$ & $-18.13(0.12)$ & $0.91(0.15)$ & $0.96(0.13)$ & $\ldots$ & $10729(1537)$ \\
\hline PTF12as & $2455933.10(0.66)$ & $-17.92(0.11)$ & $1.16(0.10)$ & $\ldots$ & $0.037(0.001)$ & $11055(2170)$ \\
\hline PTF12eci & $2456080.89(7.83)$ & $-18.70(0.18)$ & & $0.41(0.50)$ & $\ldots$ & $9678(1741)$ \\
\hline iPTF13u & $2456327.74(0.44)$ & $-18.41(0.04)$ & $0.97(0.21)$ & & $\ldots$ & 21499(2066) \\
\hline iPTF13alq & $2456394.86(2.28)$ & $-18.64(0.04)$ & $0.55(0.13)$ & $\ldots$ & $\ldots$ & 18751(1365) \\
\hline iPTF13bxl & $2456488.06(0.13)$ & $-18.90(0.10)$ & $1.12(0.09)$ & $\ldots$ & $\ldots$ & 14820(1997) \\
\hline iPTF13dnt & $2456563.75(0.93)$ & $-18.90(0.06)$ & $0.59(0.19)$ & $\ldots$ & $\ldots$ & 20821(1911) \\
\hline iPTF13ebw & $2456628.26(0.60)$ & $-17.94(0.10)$ & $1.28(0.15)$ & & $0.008(0.014)$ & $24670(1182)$ \\
\hline iPTF14dby & $2456846.66(2.28)$ & $-17.60(0.12)$ & $0.48(0.11)$ & $0.76(0.47)$ & $\ldots$ & $10708(2098)$ \\
\hline iPTF14gaq & $2456928.89(0.19)$ & $-18.35(0.07)$ & $\ldots$ & $\ldots$ & $\ldots$ & $24782(1800)$ \\
\hline iPTF15dqg & $2457340.13(0.56)$ & $-19.28(0.03)$ & $0.73(0.08)$ & & $\ldots$ & $13126(1467)$ \\
\hline iPTF15eov & $2457373.72(1.48)$ & $-21.43(0.25)$ & $0.29(0.05)$ & $0.93(0.01)$ & $\ldots$ & $17695(410)$ \\
\hline iPTF16asu & $2457524.85(0.68)$ & $-20.36(0.30)$ & $\ldots$ & $\ldots$ & $\ldots$ & $29389(882)$ \\
\hline iPTF16gox & $2457656.93(0.47)$ & $-17.51(0.31)$ & $1.00(0.09)$ & $1.07(0.68)$ & $\ldots$ & 23705(2911) \\
\hline iPTF16ilj & $2457718.90(2.06)$ & $-18.81(0.11)$ & $0.54(0.07)$ & $0.37(0.66)$ & $\ldots$ & 19341(1821) \\
\hline iPTF17cw & $2457763.88(0.75)$ & $-19.22(0.04)$ & $0.74(0.07)$ & $\ldots$ & $\ldots$ & 19526(1999) \\
\hline
\end{tabular}
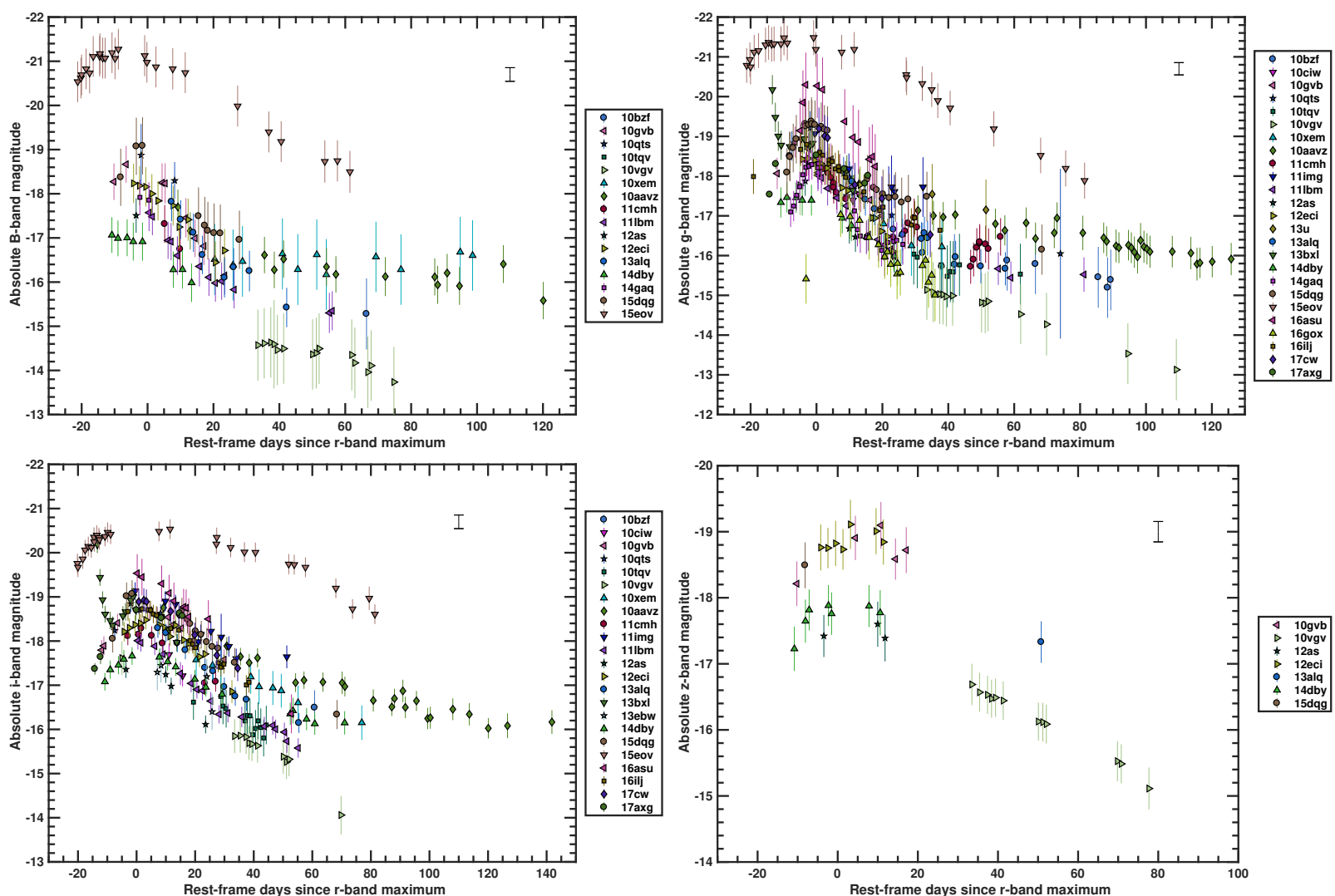

Fig. 10. Absolute Bgiz magnitudes, after (host+MW) extinction correction and K-correction. 


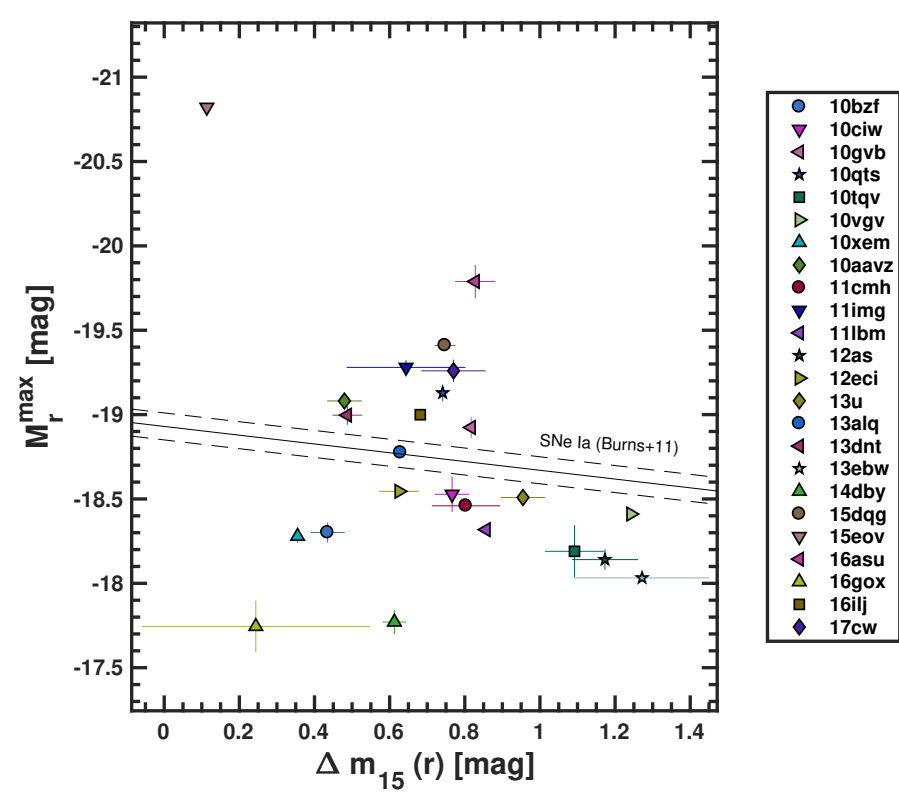

Fig. 11. Absolute peak magnitude in the $r$ band vs. $\Delta m_{15}(r)$ for the sample of 23 SNe Ic-BL. The Phillips relation from Burns et al. (2011) for the $r$ band is shown as a solid line, with the dashed lines representing the error bars. Our data show no evidence for such a relation.

provide some additional colors: $g-i, B-r$, and $B-i$. All colors tend to exhibit a sharp rise from a few days before the epoch of $r$-band peak to $\sim 20 \mathrm{~d}$ after peak, after which they start a slow decline to lower (bluer) values. This behavior becomes apparent when we fit $g-r$ with the functional formula used by Burns et al. (2014) and Stritzinger et al. (2018), and display the best fit and $3 \sigma$ uncertainties with thick red lines. The epochs before $r$-band peak are fit with a second-order polynomial. We (again) excluded iPTF15eov and iPTF16asu from the fit, as they are remarkably bluer and also display a different evolution.

To provide a continuous description of the $g-r$ color of all our SNe, we fit their individual $g-r$ colors with the function presented as a red curve in Fig. 12, using it as a template and allowing it to shift up and down. In the case of iPTF15eov, where the color evolution is clearly much slower than for the rest of the $\mathrm{SNe}$, we also stretched the color template in time by a factor of 3.125 to fit the $g-r$ color of this event. The best fits are presented in Fig. 13, and they are used to build the bolometric light curve in Sect. 6.2.

\section{Supernova spectra}

The spectra of our $\mathrm{SNe}$ are the basis for their classification as SNe Ic-BL, as discussed in Sect. 2. As shown in Table A.1, most of these 121 spectra were obtained using Keck I and P200, equipped with LRIS (Oke et al. 1995) and DBSP (Oke \& Gunn 1982), respectively. Other telescopes were also used to obtain spectra: Keck II equipped with DEIMOS (Faber et al. 2003), Gemini North with GMOS (Hook et al. 2004), Lick 3 m Shane with the Kast spectrograph (Miller \& Stone 1993), Kitt Peak National Observatory (KPNO) $4 \mathrm{~m}$ telescope with RCSpec, Hobby-Eberly Telescope (HET) with LRS (Hill et al. 1998), William Herschel Telescope (WHT) with ACAM (Benn et al. 2008), Apache Point Observatory (APO) telescope with DIS, TNG with DOLoRes, Magellan I with IMACS (Dressler et al. 2011), University of Hawaii $2.2 \mathrm{~m}$ telescope (UH88) with
SNIFS (Lantz et al. 2004), Faulkes Telescope North (FTN) with FLOYDS, Nordic Optical Telescope (NOT) with ALFOSC, P60 with SEDM (Blagorodnova et al. 2018), and Discovery Channel Telescope (DCT) with DeVeny/LMI. When possible, the spectra were obtained with an atmospheric dispersion compensator (e.g., Keck LRIS) or with the slit along the parallactic angle to help ensure accurate relative spectrophotometry (Filippenko 1982). The spectra were reduced in a standard manner, including wavelength calibration using lamp exposures and flux calibration with spectral standards observed the same night.

In the following, we present the SN spectral sequences and their properties. From the spectra we want in particular to estimate the photospheric velocities, which are later used for the modeling.

\subsection{Spectral sequences}

We present all spectra of our SNe in Figs. B.1-B.13. Each spectrum is shown in the rest frame, and its phase in rest-frame days since $r$-band maximum is reported next to it. For the SNe discovered later than $r$-band maximum, we instead report the Julian date next to each spectrum (Figs. B.12 and B.13). All spectra in these sequences have been corrected for MW and host galaxy extinction.

\subsection{Spectral temperature}

After extinction correction, we fit a blackbody (BB) function to the spectra at rest wavelengths longer than $4000 \AA$. We do not include the bluer parts since we want a temperature estimate that is not affected by the suppression of blue flux due to line blanketing. The best-fit temperatures are reported in Table A.1 and plotted in Fig. 14, where the best fits are shown by dashed red lines in the spectral-sequence figures. We fit only the spectra taken earlier than $+60 \mathrm{~d}$ after $r$-band peak, when their shape still resembles that of a blackbody. Later spectra are dominated by strong emission lines. The BB temperature evolution derived from the spectra is rather homogeneous in our sample, as shown in Fig. 14. A rapid cooling from an initial $\sim(1.5-2) \times 10^{4} \mathrm{~K}$ soon after explosion brings the average temperature at peak down to $\sim 7500 \pm 2500 \mathrm{~K}$ around the epoch of maximum light. iPTF15eov and iPTF16asu are hotter than any other SN in our sample, confirming their peculiarity.

\subsection{Photospheric velocities}

SNe Ic-BL are characterized by high expansion velocities, as implied by the broadness of their spectral lines. A good proxy for the photospheric velocity is the Fe II $\lambda 5169$ velocity, as measured by its maximum absorption position. We tried to estimate this velocity using the method illustrated by Modjaz et al. (2016). In fact, it is not easy to measure the maximum absorption position of $\mathrm{Fe}$ II $\lambda 5169$ in SNe Ic-BL, given the strong line blending in the blue part of the spectrum. The method proposed by Modjaz et al. (2016) makes use of normal SN Ic spectral templates that are shifted and smoothed to match the SN Ic-BL spectra in the region around Fe II 15169 . Before this fitting procedure, which outputs the velocity shift as compared to the expansion velocities of the SN Ic templates, the spectra of our SNe Ic-BL must be continuum-subtracted and smoothed. We therefore removed the continuum using "logwave", a software program included in the SNID package (Blondin \& Tonry 2007), and we smoothed the spectra with a fast Fourier transform (FFT) algorithm similar to that used by Modjaz et al. (2016). 

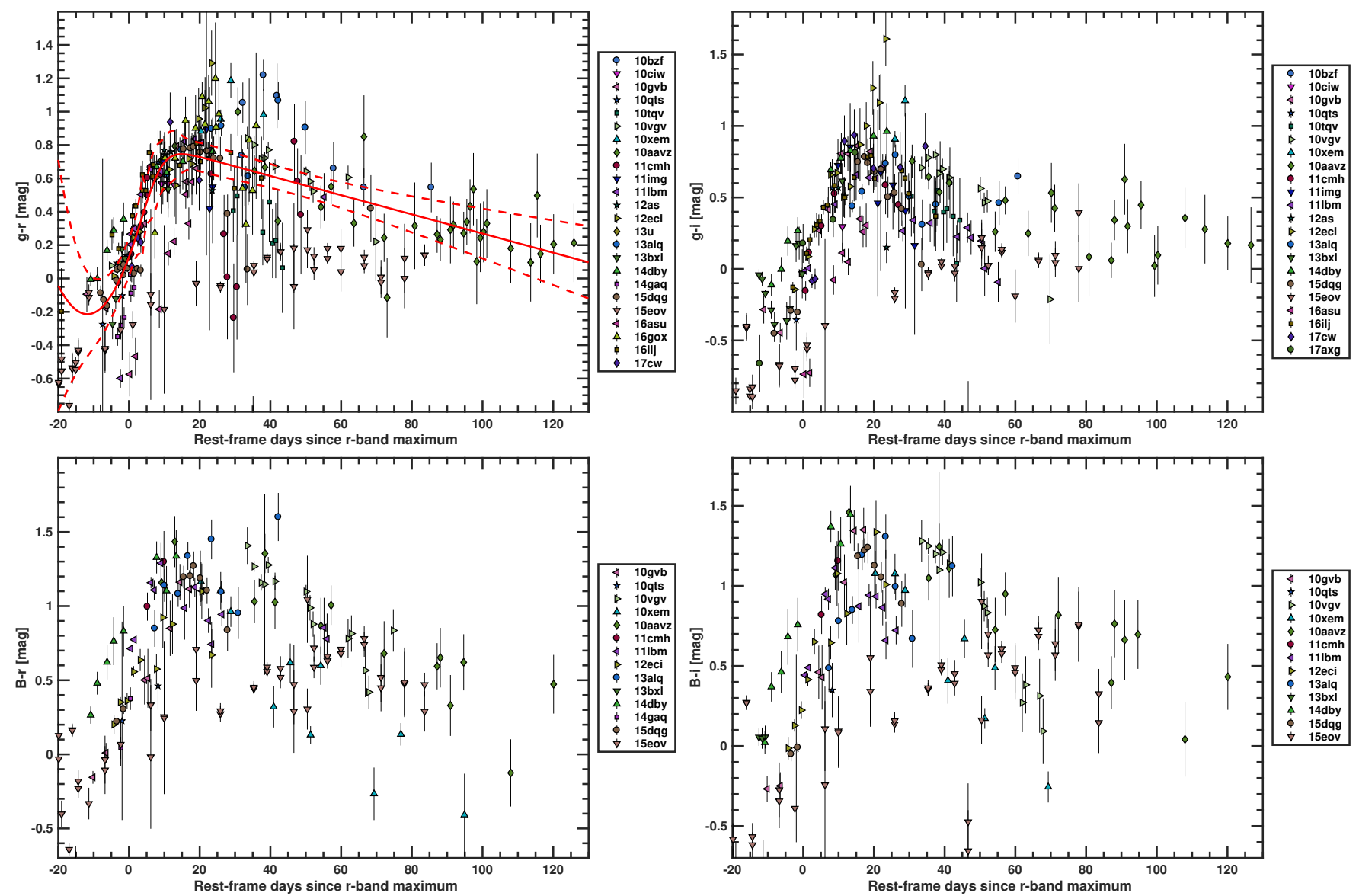

Fig. 12. $g-r, g-i, B-r$, and $B-i$ color evolution for our SNe Ic-BL. The $g-r$ color is fit with the expression used by Burns et al. (2014) and Stritzinger et al. (2018), and the best fit with its $3 \sigma$ uncertainties is shown by red lines. All of the colors are MW and host-galaxy extinction corrected.

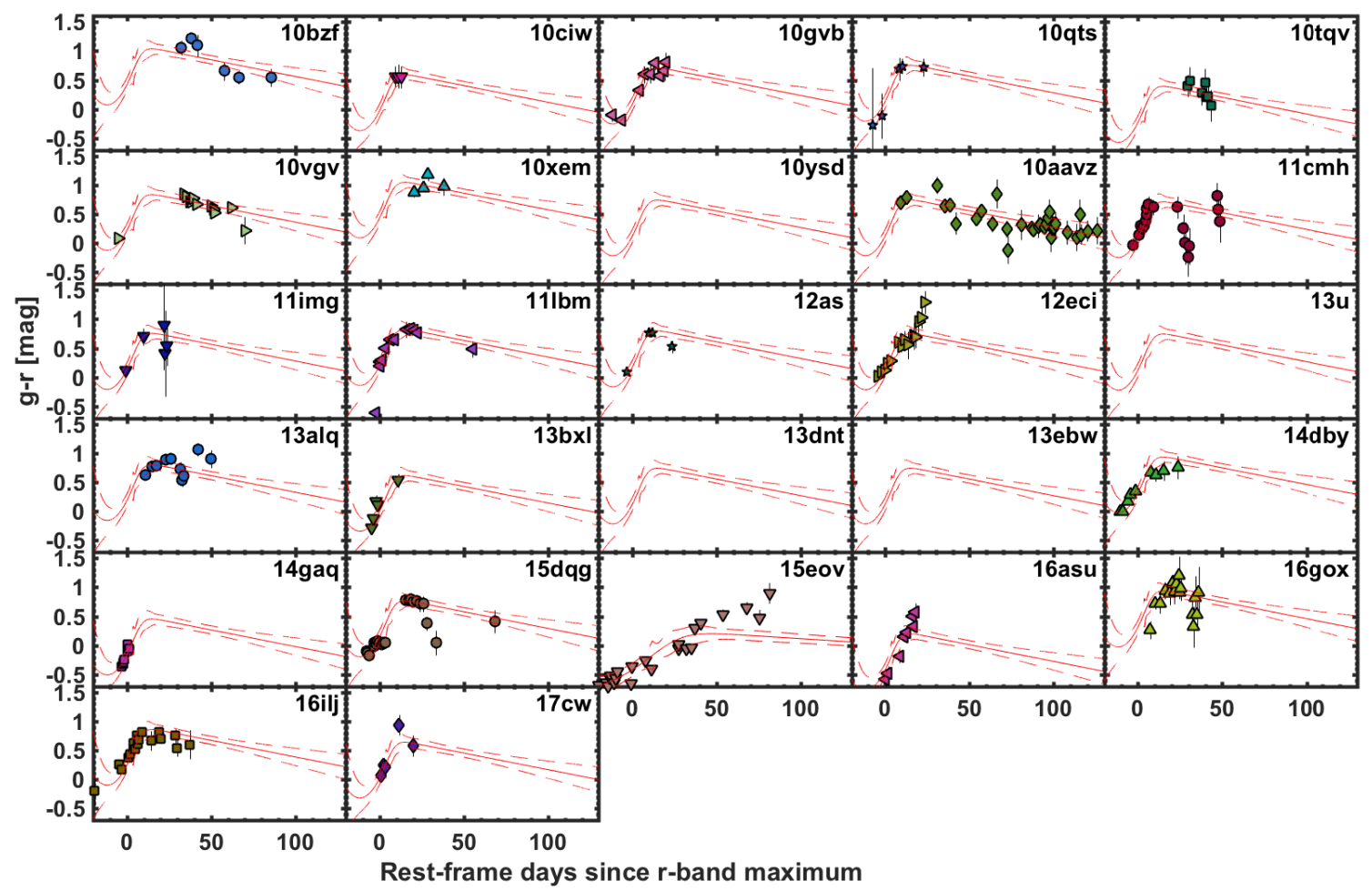

Fig. 13. Individual $g-r$ color evolution for our SNe, fit with the $g-r$ template shown in Fig. 12. In the case of PTF10ysd, iPTF13u, iPTF13dnt, and iPTF13ebw, where we have no significant $g$-band observations, we assume the template of Fig. 12 to be their $g-r$ color. 


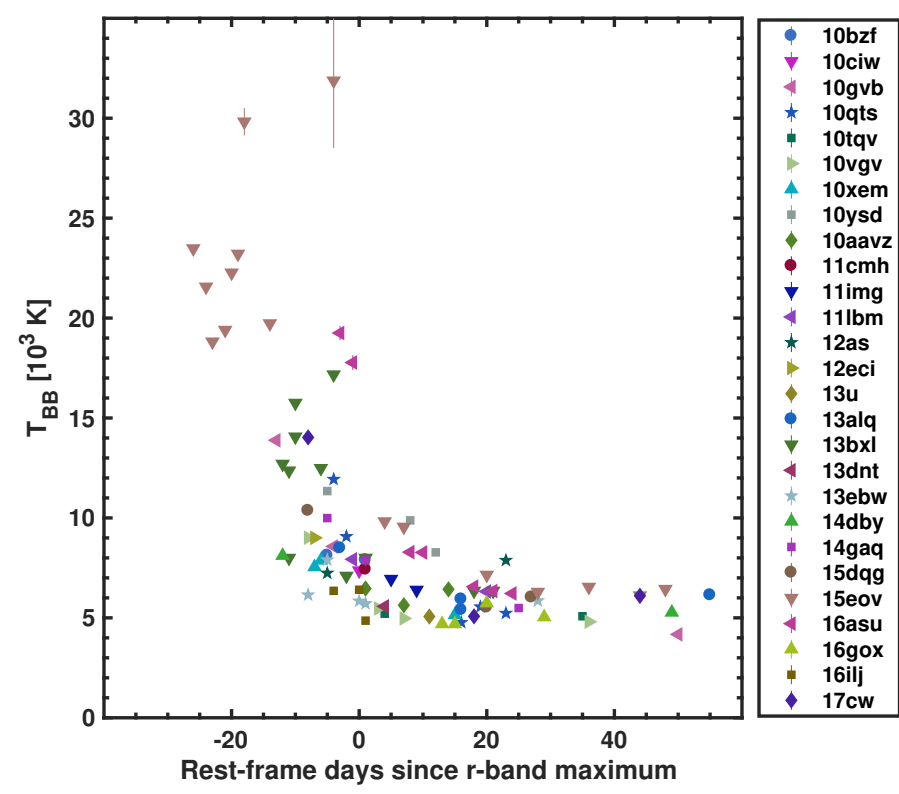

Fig. 14. Black-body temperatures from the fit to the spectral sequences.

We first confirmed that our smoothing procedure gave a similar result to those reported by Modjaz et al. (2016) by applying it to a demo spectrum already processed with the smoothing program they used. After continuum-subtracting and smoothing our spectra, we fit the spectra with the script provided by Modjaz et al. (2016) to obtain the velocity shifts with respect to the SN Ic templates at similar phases, providing the actual SN Ic-BL Fe II $\lambda 5169$ velocities in the end. We obtained the SN Ic template velocities by measuring the Fe II $\lambda 5169$ maximum velocity directly from the templates provided by Modjaz et al. (2016) and added these velocities to the shifts obtained via the spectral fit to obtain the final Fe II $\lambda 5169$ velocities for our SNe.

An example of a fit is provided in the top panel of Fig. 15. Each Fe II $\lambda 5169$ velocity measured for each SN is shown in the bottom panel of Fig. 15, as well as in the lower subpanels of Fig. 20. These velocities are also reported in Table A.1, along with the information about the spectra of our SNe. In the bottom panel of Fig. 15, our velocities are compared to those of other SNe Ic-BL from Modjaz et al. (2016). With the exception of iPTF15eov, which exhibits a high velocity for a relatively long time, the velocity trend for our $\mathrm{SNe}$ is similar to that found for the SNe discussed by Modjaz et al. (2016).

Since we need the velocities at peak brightness for deriving properties from the light-curve models (see Sect. 6), we first fit the SN Ic-template velocities with a polynomial and then use this polynomial (with its normalization left as a free parameter) to fit the velocity profiles of our SNe. The best fits are shown by solid lines in the bottom panels of Fig. 20, and the interpolated velocities at $r$-band peak are shown by empty diamonds. The uncertainty in the velocity at peak is estimated by taking the standard deviation of the peak velocities derived from 100 Monte Carlosimulated velocity profiles (based on the uncertainties of each velocity measurement) fitted with the previous velocity template. The values of the photospheric velocities at the epoch of $r$-band peak for our SNe are given in Table 3.

\subsection{Spectral signatures of different viewing angles of jet-related explosions}

Barnes et al. (2018) have predicted that if the explosion of an $\mathrm{SN}$ Ic-BL is characterized by the presence of a central engine that produces a jet, then the SN spectra and light curves should depend on the viewing angle. In particular, a polar point of view would give higher velocities at early epochs and redder spectra at later epochs (see Barnes et al. 2018, Fig. 5), as compared to an equatorial viewing angle. In our SN sample, we found a few examples where a SN that displays higher velocities than another $\mathrm{SN}$ at early epochs also turns out to be redder than that $\mathrm{SN}$ at late epochs, in line with the models provided by Barnes et al. (2018). However, when we systematically test the late-time colors versus the early-time velocity for the entire sample, there is no clear evidence that the faster events are also redder at late epochs.

\section{Bolometric properties}

We next want to determine the bolometric properties of our $\mathrm{SNe}$ to model them and derive progenitor and explosion parameters. We therefore require estimates of the explosion epochs and bolometric light curves, in addition to the photospheric velocity profiles, which we obtained in Sect. 5.3.

\subsection{Explosion epochs and rise times}

In order to estimate the explosion epochs, we fit the $r$-band light curves of our SNe with the $R$-band light curve of SN 1998bw, the prototypical SN Ic-BL (see Cano 2013, for details about the fit to the light curve of SN 1998bw.) For SN 1998bw, the epoch of explosion is set equal to the time of GRB 980425. The light curve of SN 1998bw is shifted in magnitude and stretched in time until it fits our SN light curves at early epochs; that is, until $+30 \mathrm{~d}$ post peak. Since the epochs of explosion and $R$-band maximum for SN 1998bw are known, the temporal stretch of the best fit allows us to infer the explosion epochs of our $\mathrm{SNe}$. We check the estimates against the pre-explosion upper limits and, with the exception of three cases, these estimates are consistent. When not consistent, we assume the last nondetection as the explosion epoch. We note that, in the special case of iPTF16asu (Whitesides et al. 2017), whose peculiar light-curve shape (characterized by a very fast rise) cannot be properly reproduced by stretching SN 1998bw, we take a value from the literature. We furthermore checked this method against iPTF13bxl, whose explosion epoch is known thanks to the associated GRB, and the results are consistent within $3.6 \mathrm{~d}$, but for this event we had to modify the light curve to subtract the bright afterglow (see Fig. 5), and this introduces more uncertainty in the light-curve fit. We adopt $\pm 2 \mathrm{~d}$ as the uncertainty in the explosion epochs.

The best fits and the obtained explosion epochs are shown in Fig. 16. The values of the inferred explosion epochs are reported in Table 2. The explosion epochs and the epochs of $r$-band maximum allow us to compute the rest-frame $r$-band rise time; the distribution is plotted in Fig. 17. We note that the average $r$-band rise time $(15 \pm 6 \mathrm{~d})$ is consistent with that computed by Taddia et al. (2015) for the SDSS SNe Ic-BL (14.7d). In the same figure, we also plot the rise times for the bolometric light curves, which we discuss further in Sect. 6.2. The bolometric rise times are similar to those in the $r$ band and on average only $1.6 \mathrm{~d}$ shorter. This was also observed in the SDSS SN Ibc sample (Taddia et al. 2015), where the bolometric rise times are also slightly shorter than the $r$ rise times. The $r$-band rise time obviously correlates with $\Delta m_{-10}$ but does not correlate with $\Delta m_{15}$ in the same band. 

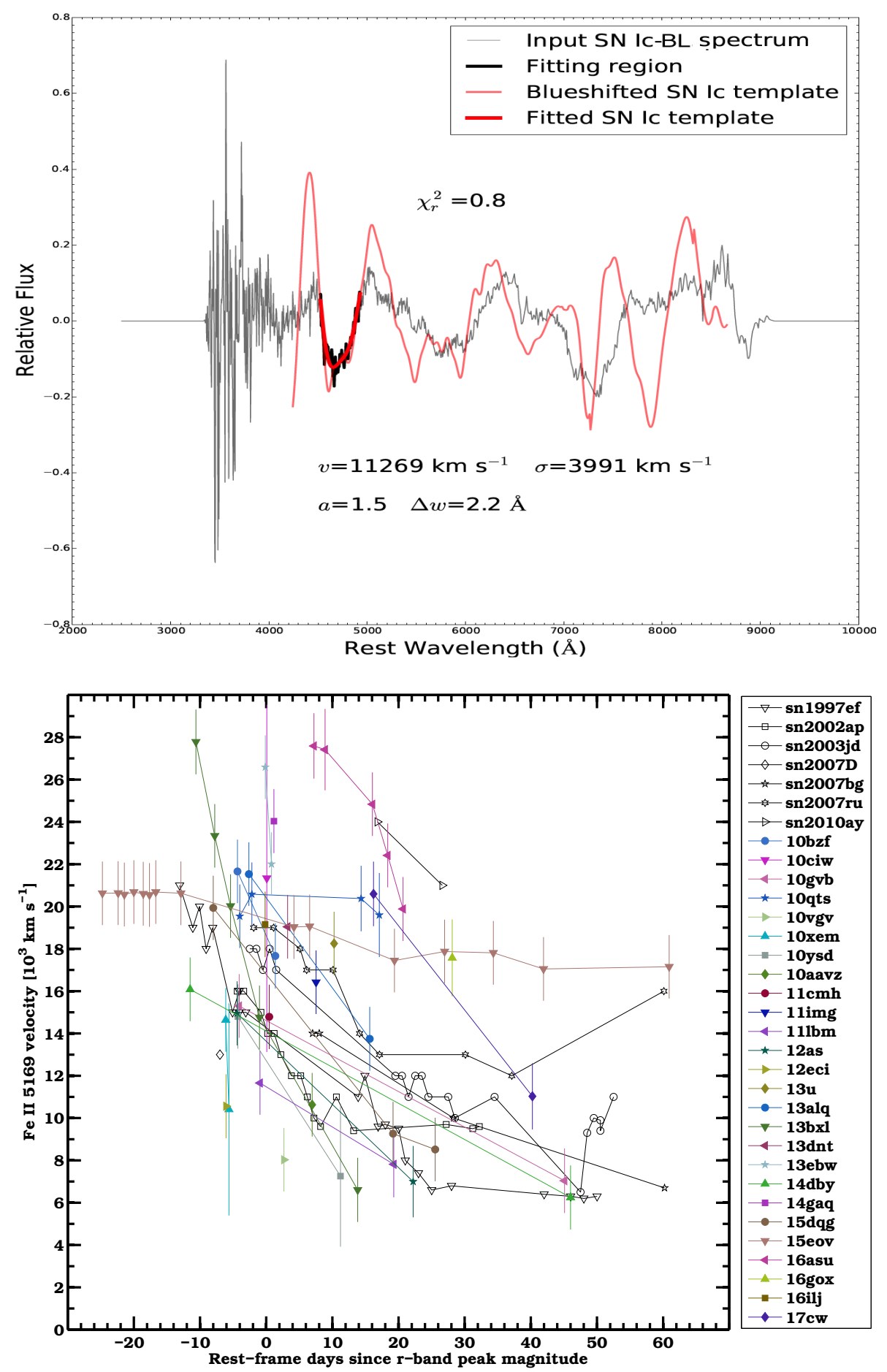

Fig. 15. Top panel: spectral template fit to a spectrum of PTF10bgz, as obtained from the Modjaz et al. (2016) routine. The SN Ic template (thin red line) has been shifted and smoothed to match the spectrum of our SN (gray line) in the wavelength region around $\mathrm{Fe}$ II $\lambda 5169$ (black line). The best fit is shown as a thick red line. The shift velocity $v$ must be added to the known Fe II $\lambda 5169$ velocity of the SN Ic template to obtain the Fe II $\lambda 5169$ velocity of our SN. Bottom panel: velocity of our SN Ic-BL sample as compared to the SNe Ic-BL (not associated with GRBs) from Modjaz et al. (2016).

\subsection{Bolometric light curves}

Given the lack of complete multiband coverage, especially at early epochs, we resort to use the absolute $r$-band light curves (Fig. 9) and the individual fits to the $g-r$ colors shown in Fig. 13 to compute the bolometric light curves. This is done using the bolometric corrections for SE SNe presented by Lyman et al. (2014), as also tested by Taddia et al. (2018a) on a large sample of SE SNe from the Carnegie Supernova Project. In this way, we can estimate bolometric light curves covering the rising phase as well. We present the final bolometric light curves in Fig. 18 (lefthand panel), as a function of days since explosion. The uncertainty associated with each bolometric light-curve point in the figure does not include the systematic error due to the bolometric correction $(0.076 \mathrm{mag})$ or to the uncertainty in the distance $(0.04 \mathrm{mag})$, but it includes the uncertainties in the photometry, extinction correction, and $g-r$ template.

We fit the bolometric light curves with the same function used to fit the $r$ light curves and plot the best fits as solid lines. As in the case of the $r$ light curves, the best fits allow us to measure some properties of the bolometric light curves, such as the peak epoch and magnitude, $\Delta m_{15}, \Delta m_{-10}$, and the linear decay slope. In Table 3, we report all these parameters.

Our $\mathrm{SNe}$ range in bolometric peak absolute magnitude between -17.5 and $-21.4 \mathrm{mag}$; the range is greater than that in the $r$ band, as iPTF15eov has a large fraction of its bolometric 


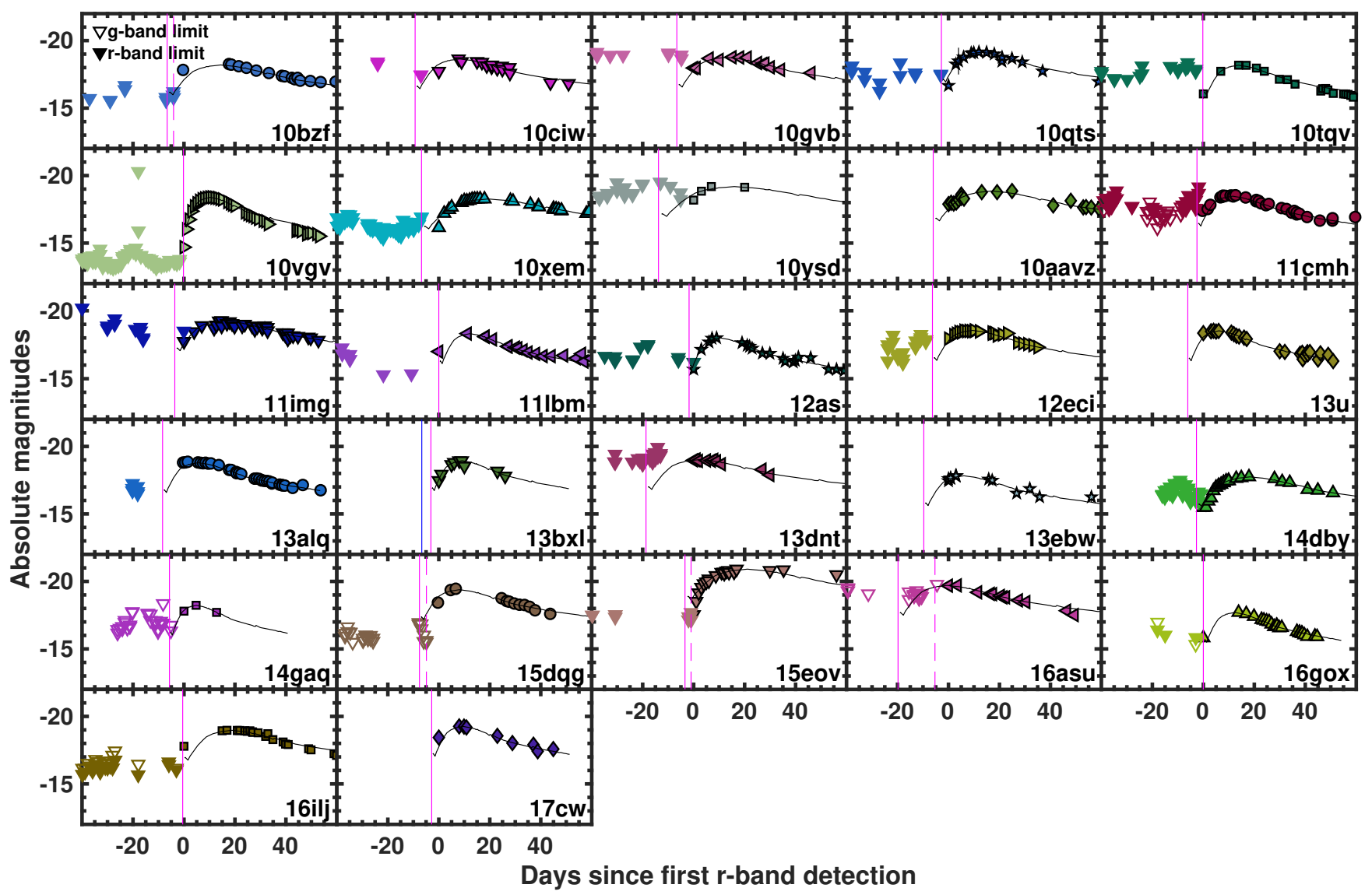

Fig. 16. Fits of the $r$ light curves of our SNe Ic-BL with the stretched light curve of SN 1998bw (black solid line). This fitting aims at determining the explosion epoch, marked by a vertical magenta line. The pre-explosion limits, marked by empty ( $g$-band) and filled ( $r$-band) triangles, are in agreement with the computed explosion epochs, except in four cases where we instead adopt the last nondetection as the explosion epoch and a value from the literature for iPTF16asu (magenta dashed lines). For iPTF13bxl, we adopt the time of the detected GRB as the explosion epoch (blue vertical line).

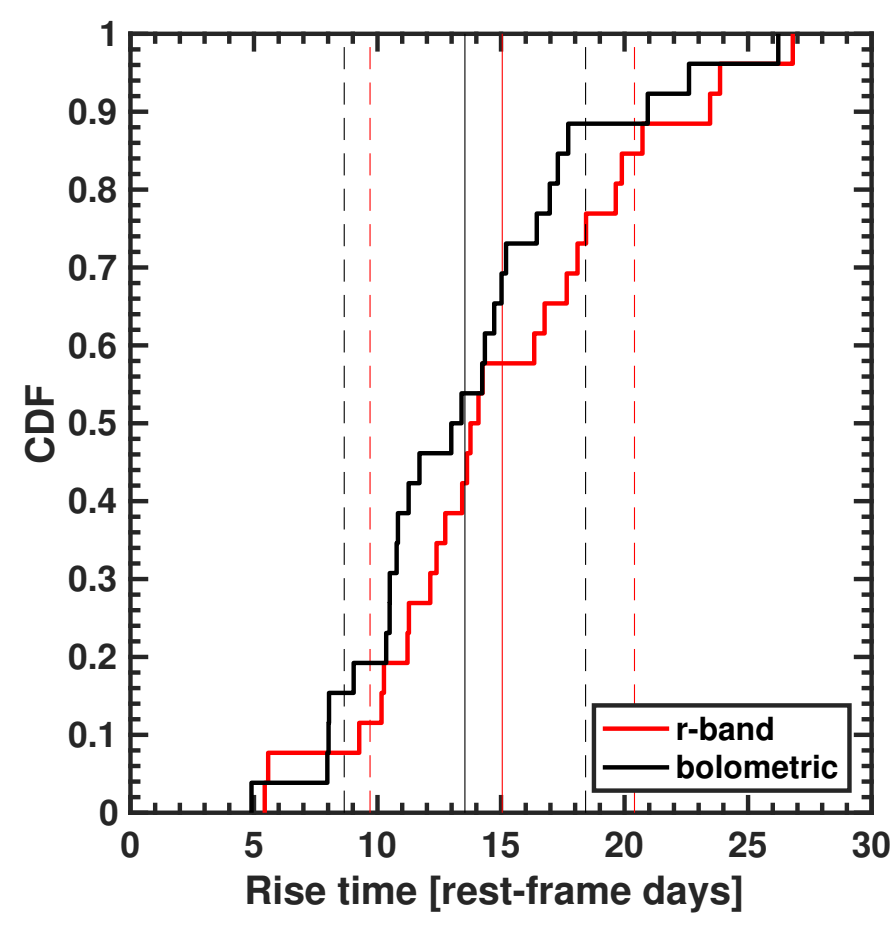

Fig. 17. Rest-frame rise-time distributions for the $r$-band (red) and the bolometric (black) light curves. The averages are marked by solid vertical lines, and their standard deviations as dashed vertical lines. emission in the bluer bands. In our SNe sample, iPTF15eov is the most luminous, followed by iPTF16asu. These two SNe have the longest and shortest bolometric rise times, respectively. The distribution of the peak absolute bolometric magnitudes is shown in the right-hand panel of Fig. 18. If we include iPTF15eov and iPTF16asu, the average bolometric peak is $-18.7 \pm 0.8 \mathrm{mag}$, whereas if we exclude these peculiar objects, the average bolometric peak is $-18.5 \pm 0.5 \mathrm{mag}$. As with $r$, we find no correlation between peak magnitude and $\Delta m_{15}$. Like in the $r$ band, the bolometric rise time obviously correlates with $\Delta m_{-10}$. The correlation between $\Delta m_{-10}$ and $\Delta m_{15}$ is present but weaker than in $r$, as we have fewer points. The individual bolometric light curves are also shown in the subpanels of Fig. 20.

\subsection{Blackbody temperatures and radii}

Having multiband photometry, we can investigate how the BB temperature corresponding to the spectral energy distribution (SED) changes with time for each $\mathrm{SN}$. We interpolate the K-corrected $g$ and $r$ photometry to each epoch of $i$-band data. The extinction-corrected gri magnitudes are converted into fluxes at the effective wavelength of each filter, and for each epoch we fit the SED with a BB function. In the bottom panel of Fig. 19, the temperature shows a progressive decline from the explosion date until $\sim 25 \mathrm{~d}$, when it flattens out at $\sim 6000 \mathrm{~K}$, in line with what was found by Taddia et al. (2015, 2018a) for $\mathrm{SE} \mathrm{SNe}$. We note that the temperature values also exhibit a 

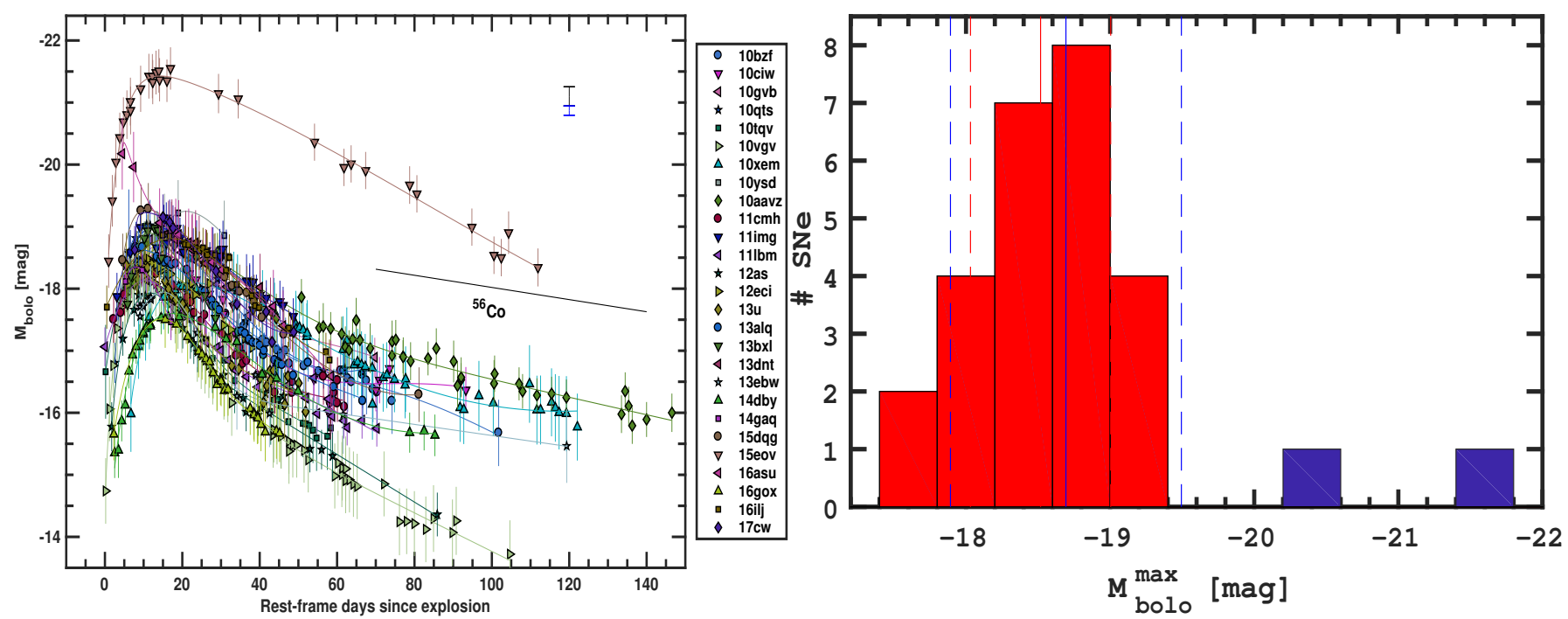

Fig. 18. Left panel: bolometric light curves, after (host $+\mathrm{MW}$ ) extinction correction and K-correction. The solid lines are the best fits of the function also used to fit the $r$ band. The systematic uncertainties in the distance (black segment) and in the bolometric correction (blue segment) are shown in the upper-right corner. Right panel: distribution of the absolute bolometric peak magnitudes for our sample. iPTF15eov and iPTF16asu are marked in blue. Blue vertical lines mark the average and the standard deviation of the distribution when including all of the objects. Red vertical lines mark the average and the standard deviation of the distribution when excluding iPTF15eov and iPTF16asu.

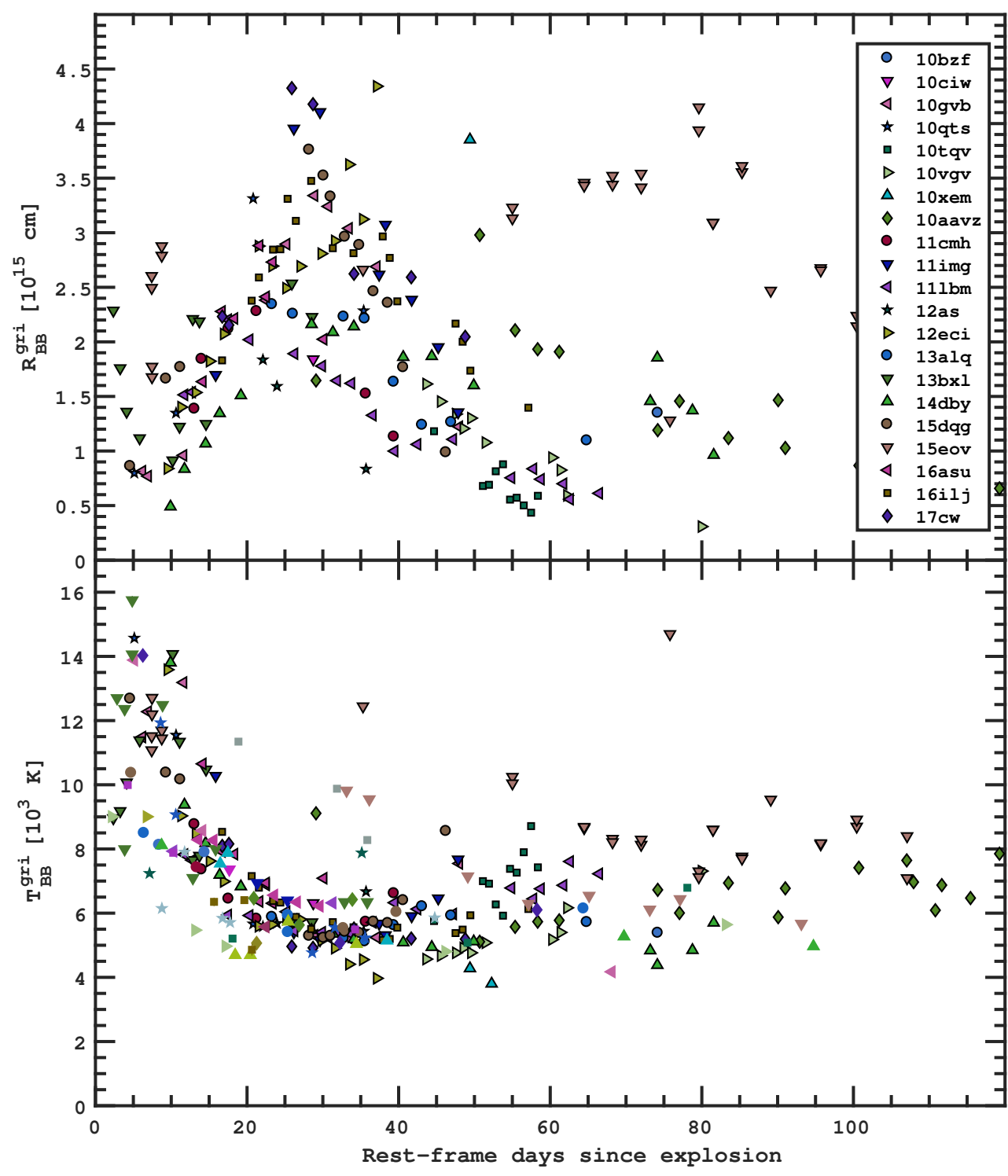

Fig. 19. BB radius (top panel) and temperature (bottom panel) from the fit of the gri SEDs of our SNe (symbols with black marker edges). We also report the temperatures from the spectral BB fits already reported in Fig. 14 (markers without black edges), which overall match the evolution of temperature from the gri SED fits. 


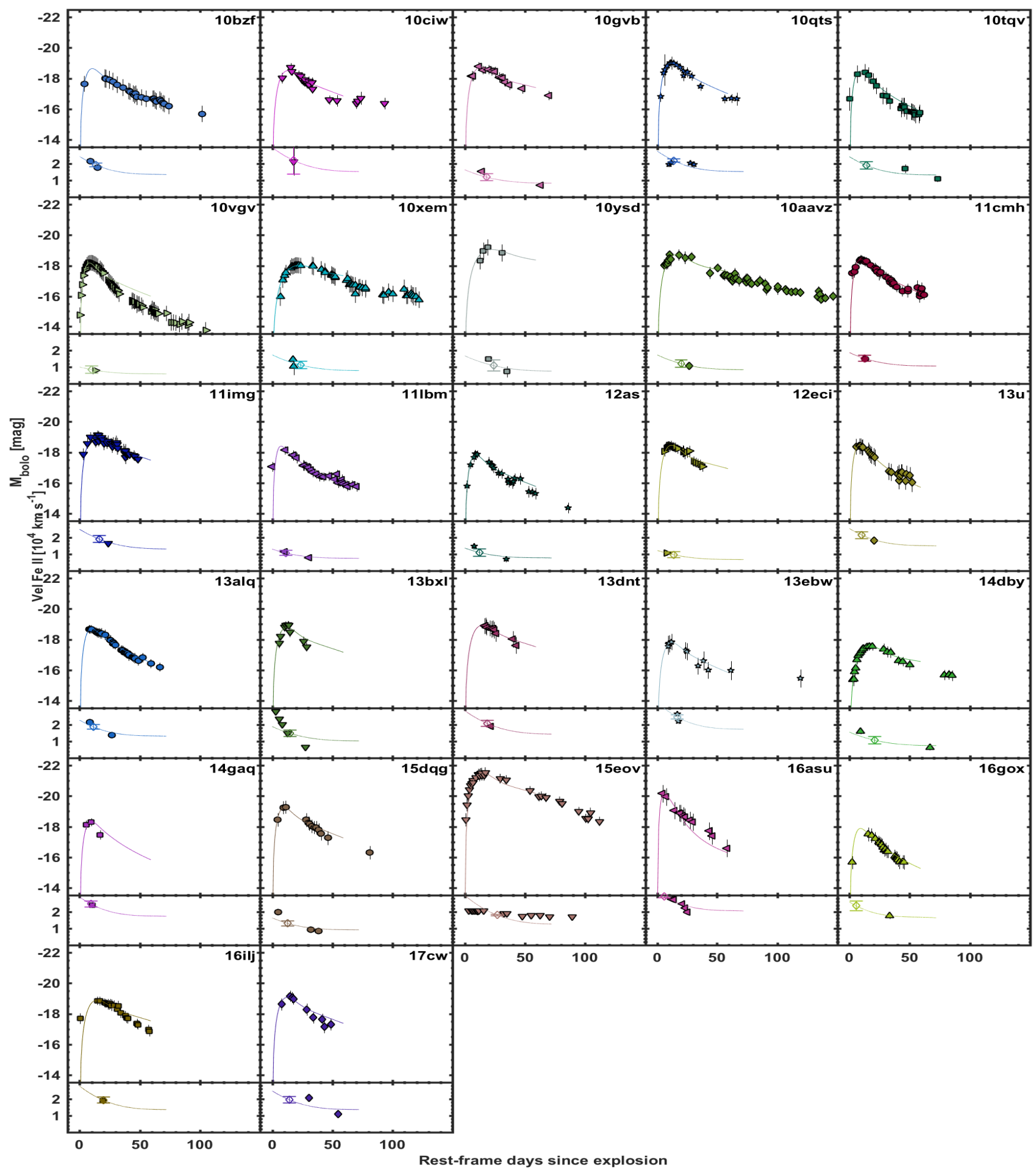

Fig. 20. Bolometric light curves and velocity profiles for our sample. For the SNe without velocity measurements, we assumed the average velocity profile. The bolometric light curves were fit with the Arnett model, shown as a solid line. The velocity profiles were fit with a scaled polynomial that also reproduces the velocity profile of the SN Ic spectral templates provided by Modjaz et al. (2016), and it is shown with solid lines in the velocity subpanels. The velocity at peak brightness is marked with an empty diamond.

relatively small spread as a consequence of how we computed the host-extinction corrections; that is, assuming the same $g-r$ colors between 0 and $20 \mathrm{~d}$ after $r$-band peak. With the BB fit, we also obtain the $\mathrm{BB}$ radius, which is shown in the top panel of Fig. 19. The radius increases up to $\sim 30 \mathrm{~d}$ since explosion and then slowly decreases, again consistent with previous findings for SE SNe (Taddia et al. 2015, 2018a).

\section{Progenitor and explosion parameters}

We fit the bolometric light curves with an Arnett model (Arnett 1982), as done by Taddia et al. (2018a). This includes the possibility of escaping gamma rays. We restrict our fits to the early epochs of the light curves, when the $\mathrm{SNe}$ are in their photospheric phases (i.e., $\lesssim 60 \mathrm{~d}$ after peak brightness). From this 
Table 4. Explosion parameters from Arnett models and from hydrodynamical model scaling relations.

\begin{tabular}{|c|c|c|c|c|}
\hline Supernova & $\begin{array}{c}M_{\mathrm{ej}} \\
\left(M_{\odot}\right)\end{array}$ & $\begin{array}{c}E_{K} \\
\left(10^{51} \text { erg }\right) \\
\end{array}$ & $\begin{array}{c}M\left({ }^{56} \mathrm{Ni}\right) \\
\left(M_{\odot}\right)\end{array}$ & $\begin{array}{c}{ }^{56} \mathrm{Ni} \text { mixing } \\
\left(\% M_{\mathrm{ej}}\right)\end{array}$ \\
\hline PTF10bzf & $2.8(0.7)$ & $6.2(0.7)$ & $0.274(0.125)$ & 100 \\
\hline PTF10ciw & $5.7(8.4)$ & $16.5(12.3)$ & $0.292(0.097)$ & 100 \\
\hline PTF10gvb & $3.3(2.2)$ & $2.8(0.9)$ & $0.371(0.120)$ & 100 \\
\hline PTF10qts & $2.9(0.5)$ & $8.3(0.7)$ & $0.392(0.137)$ & 100 \\
\hline PTF10tqv & $1.6(0.8)$ & $3.5(0.9)$ & $0.203(0.093)$ & 100 \\
\hline PTF10vgv & $0.6(0.6)$ & $0.3(0.1)$ & $0.149(0.067)$ & 100 \\
\hline PTF10xem & $11.1(8.9)$ & $8.3(3.4)$ & $0.312(0.142)$ & 93 \\
\hline PTF10ysd & $10.4(12.7)$ & $7.4(4.5)$ & $0.802(0.370)$ & 94 \\
\hline PTF10aavz & $5.0(3.5)$ & $4.3(1.5)$ & $0.424(0.141)$ & 100 \\
\hline PTF11cmh & $1.7(0.8)$ & $2.3(0.6)$ & $0.205(0.070)$ & 100 \\
\hline PTF11img & $5.2(2.2)$ & $11.3(2.4)$ & $0.479(0.170)$ & 100 \\
\hline PTF11lbm & $0.6(0.4)$ & $0.4(0.1)$ & $0.172(0.056)$ & 100 \\
\hline PTF12as & $1.2(0.9)$ & $0.8(0.3)$ & $0.117(0.039)$ & 100 \\
\hline PTF12eci & $1.9(1.3)$ & $1.0(0.4)$ & $0.253(0.083)$ & 87 \\
\hline iPTF13u & $1.8(0.7)$ & $4.9(0.9)$ & $0.199(0.091)$ & 100 \\
\hline iPTF13alq & $2.4(0.7)$ & $5.1(0.7)$ & $0.281(0.090)$ & 100 \\
\hline iPTF13bxl & $3.1(1.7)$ & 4.1(1.1) & $0.354(0.109)$ & $\ldots$ \\
\hline iPTF13dnt & $7.0(2.6)$ & $18.2(3.3)$ & $0.469(0.211)$ & 90 \\
\hline iPTF13ebw & $5.3(1.0)$ & $19.1(1.8)$ & $0.142(0.065)$ & 94 \\
\hline iPTF14dby & $5.1(4.0)$ & $3.5(1.4)$ & $0.155(0.050)$ & 100 \\
\hline iPTF14gaq & $3.0(0.9)$ & $11.0(1.6)$ & $0.195(0.060)$ & 100 \\
\hline iPTF15dqg & $1.7(0.8)$ & $1.7(0.4)$ & $0.461(0.184)$ & 100 \\
\hline iPTF15eov & $6.1(0.5)$ & $11.3(0.5)$ & $5.114(1.773)$ & \\
\hline iPTF16asu & $0.9(0.1)$ & $4.8(0.3)$ & $0.763(0.355)$ & $\cdots$ \\
\hline iPTF16gox & $2.4(1.2)$ & $8.2(2.0)$ & $0.123(0.049)$ & 98 \\
\hline iPTF16ilj & $6.6(2.5)$ & $14.8(2.8)$ & $0.462(0.154)$ & 90 \\
\hline iPTF17cw & $4.5(1.8)$ & $10.2(2.1)$ & $0.473(0.174)$ & 100 \\
\hline$\langle$ Ic-BL $\rangle$ & $3.9(2.7)$ & $7.1(5.4)$ & $0.506(0.938)$ & \\
\hline Median Ic-BL & 3.1 & 5.1 & 0.292 & \\
\hline$\langle\mathrm{Ic}-\mathrm{BL}\rangle^{a}$ & $3.9(2.8)$ & $7.0(5.6)$ & $0.310(0.160)$ & \\
\hline$\langle\mathrm{Ic}-\mathrm{BL}\rangle^{b}$ & $3.9(2.8)$ & 7.1(5.7) & $0.309(0.164)$ & \\
\hline
\end{tabular}

Notes. Uncertainties in the averages are the standard deviations. ${ }^{(a)}$ Computed without iPTF15eov and iPTF16asu. ${ }^{(b)}$ Computed without iPTF15eov, iPTF16asu, and GRB SN iPTF13bxl.

modeling, it is possible to obtain estimates of the ${ }^{56} \mathrm{Ni}$ mass, $M\left({ }^{56} \mathrm{Ni}\right)$, kinetic energy of the explosion $\left(E_{K}\right)$, and ejecta mass $\left(M_{\mathrm{ej}}\right)$. We use $E / M=(3 / 10) v^{2}$ assuming the SN ejecta are spherical with a uniform density, where $v$ is equal to the velocity at $r$-band peak as measured in Sect. 5.3. We also assume constant opacity $\kappa=0.07 \mathrm{~cm}^{2} \mathrm{~g}^{-1}$. This is likely not completely correct, but it allows for comparisons with other works on SE SN samples. We also note that in Taddia et al. (2018a) such an assumption provided similar results to more sophisticated hydrodynamical models.

The obtained values for $M_{\mathrm{ej}}, E_{K}$, and $M\left({ }^{56} \mathrm{Ni}\right)$ are reported in Table 4. The uncertainties in $M_{\mathrm{ej}}$ and $E_{K}$ depend mainly on the uncertainty in the expansion velocity, whereas that in $M\left({ }^{56} \mathrm{Ni}\right)$ is mostly due to the uncertainty in the extinction, but also in the SN distance; the systematic uncertainty due to the bolometric corrections used in Sect. 6.2 is significant as well. We obtained averages $\left\langle M_{\mathrm{ej}}\right\rangle=3.9 \pm 2.7 M_{\odot},\left\langle E_{K}\right\rangle=(7.1 \pm$ 5.4) $\times 10^{51} \mathrm{erg}$, and $\left\langle M\left({ }^{56} \mathrm{Ni}\right)\right\rangle=0.51 \pm 0.94 M_{\odot}$. If we remove iPTF16asu and iPTF15eov, we obtain $\left\langle M_{\mathrm{ej}}\right\rangle=3.9 \pm 2.8 M_{\odot}$, $\left\langle E_{K}\right\rangle=(7.0 \pm 5.6) \times 10^{51} \mathrm{erg}$, and $\left\langle M\left({ }^{56} \mathrm{Ni}\right)\right\rangle=0.31 \pm 0.16 M_{\odot}$. If we also remove the GRB SN iPTF13bxl, considering only the regular SNe Ic-BL, we obtain $\left\langle M_{e j}\right\rangle=4.0 \pm 2.9 M_{\odot}$, $\left\langle E_{K}\right\rangle=(7.0 \pm 5.8) \times 10^{51} \mathrm{erg}$, and $\left\langle M\left({ }^{56} \mathrm{Ni}\right)\right\rangle=0.31 \pm 0.17 M_{\odot}$.
Removing the two outliers significantly reduces the average value of $M\left({ }^{56} \mathrm{Ni}\right)$. The averages of the other parameters are similar. The GRB SN parameters do not significantly alter the averages.

In Fig. 21, we plot each parameter against the others, identifying a correlation between $M_{\mathrm{ej}}$ and $E_{K}$ (see bottom panel), a weaker correlation between $M_{\mathrm{ej}}$ and $M\left({ }^{56} \mathrm{Ni}\right)$ (top panel), and between $M\left({ }^{56} \mathrm{Ni}\right)$ and $E_{K}$ (central panel). This was also observed for SE SNe by Lyman et al. (2016) and Taddia et al. (2018a).

iPTF15eov and iPTF16asu show a very large ratio $(>80 \%)$ of $M\left({ }^{56} \mathrm{Ni}\right)$ over $M_{\mathrm{ej}}$, which indicates that they do not fit the traditional radioactive-powered scenario, as already suggested by their peculiar light curves. As such, the values derived for these objects from the above method are not valid.

The probability distributions of the three aforementioned parameters are plotted in Fig. 22. We excluded GRB SNe, as well as iPTF15eov and iPTF16asu, for further comparison with literature data on regular SNe Ic-BL. The scatter of these three parameters is larger than the typical uncertainties for each individual $\mathrm{SN}$, so there is an intrinsic range of values in the SN Ic-BL family for $M_{\mathrm{ej}}, E_{K}$, and $M\left({ }^{56} \mathrm{Ni}\right)$.

An interesting property of SNe powered by radioactive decay is the distribution of the radioactive ${ }^{56} \mathrm{Ni}$. The degree of ${ }^{56} \mathrm{Ni}$ 

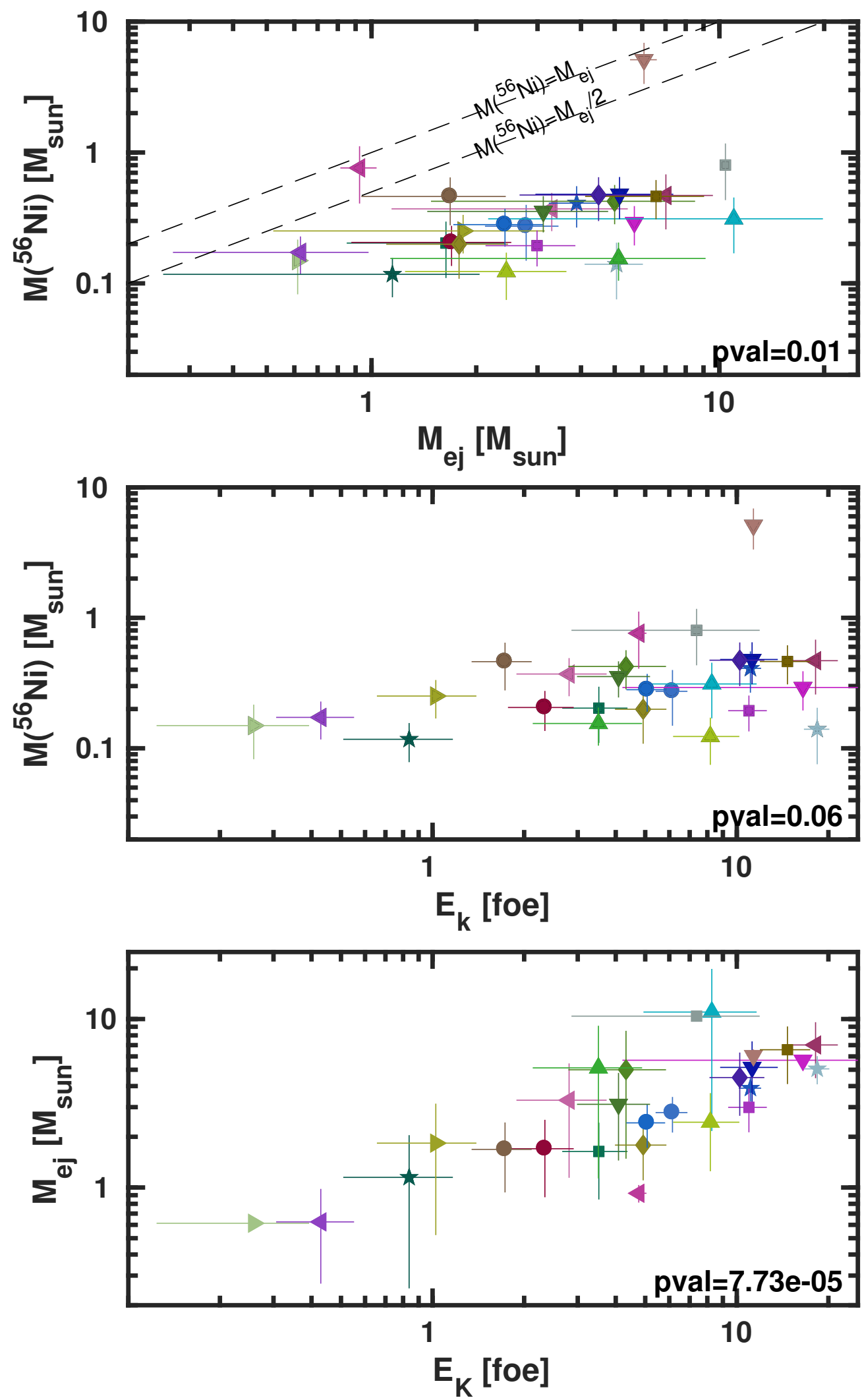

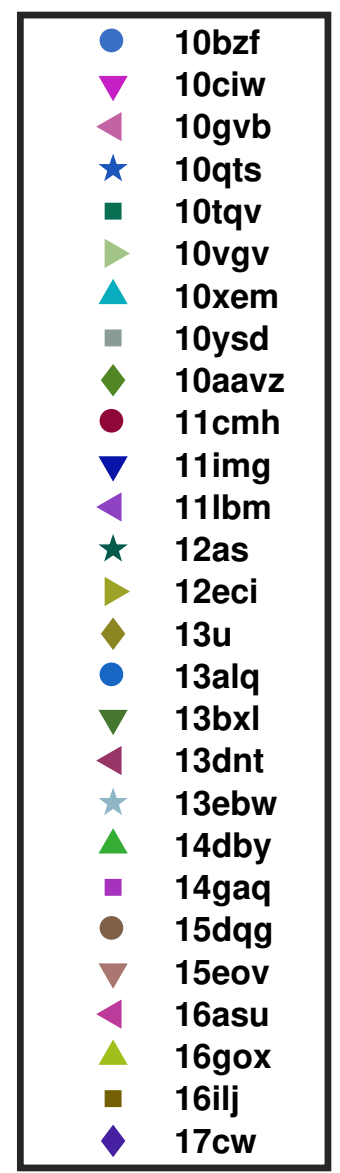

Fig. 21. Explosion and progenitor parameters plotted against each other for our SN sample.

mixing in the ejecta affects the light-curve shape, and it is possible to estimate its extent by comparison with theoretical models. Arnett's model assumes that all of the ${ }^{56} \mathrm{Ni}$ is in the center of the ejecta, and therefore we turned to more sophisticated hydrodynamical models to derive scaling relations aimed to estimate the degree of ${ }^{56} \mathrm{Ni}$ mixing. We followed an approach similar to that used by Taddia et al. (2016a) to determine the progenitor parameters of SN 1987A-like events from iPTF. We first built a realistic progenitor star for SNe Ic-BL, which is a moderately low final mass $\left(7.34 M_{\odot}\right)$, He-poor $\left(0.56 M_{\odot}\right.$ of $\left.\mathrm{He}\right)$, 

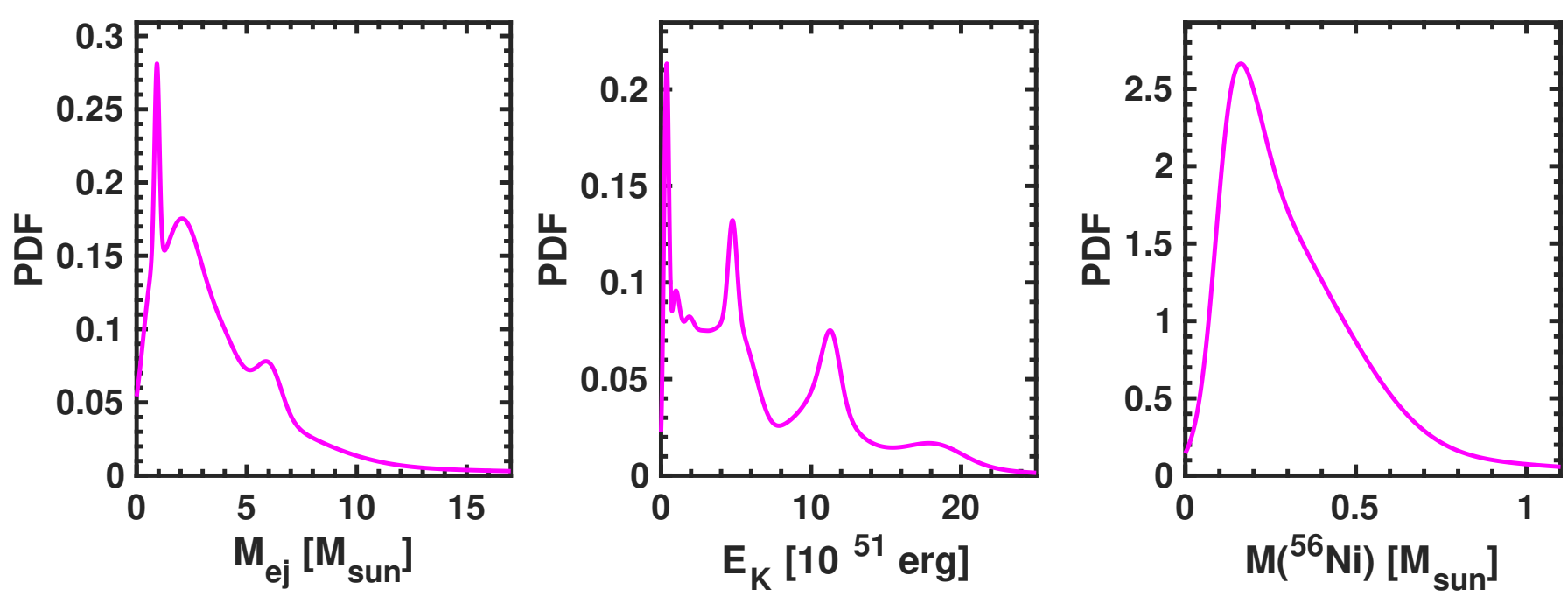

Fig. 22. Probability density functions for the three main explosion parameters for our sample of SNe Ic-BL, excluding GRB-SNe as well as the peculiar SNe iPTF15eov and iPTF16asu.
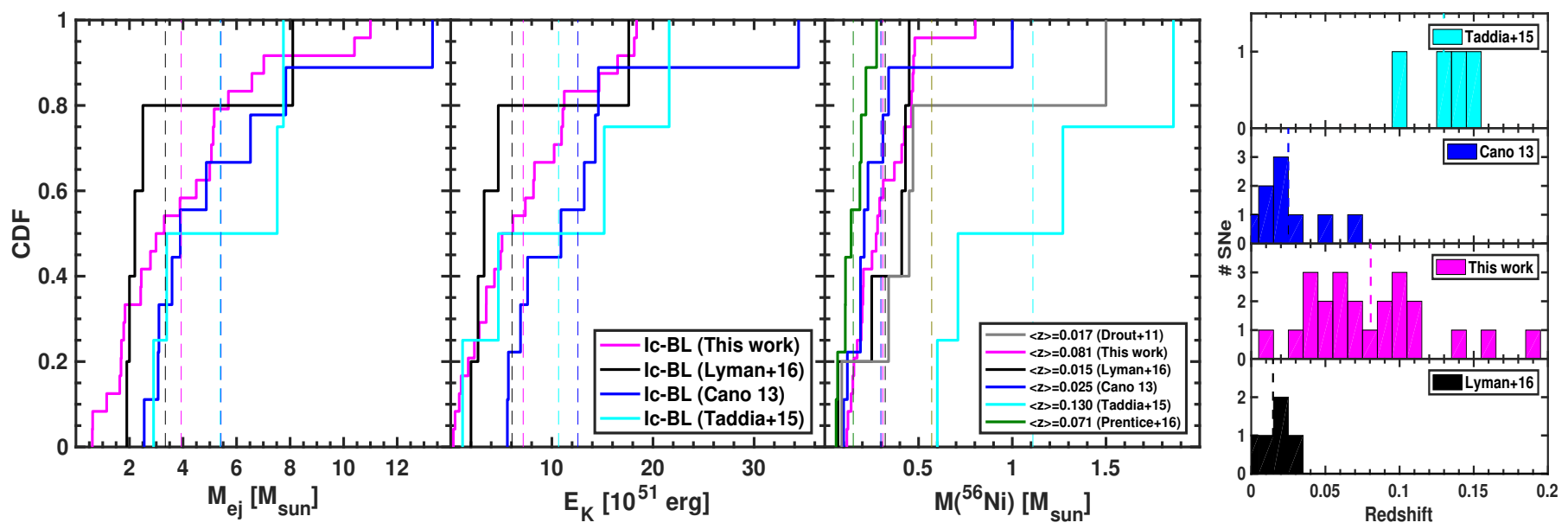

Fig. 23. Cumulative probability functions for the three main explosion parameters as compared to those of other SN Ic-BL samples in the literature.

compact $\left(R=10 R_{\odot}\right)$ star. This was carried out using MESA (Paxton et al. 2011), evolving a star with initial mass $85 M_{\odot}$, fast rotation velocity $\left(350 \mathrm{~km} \mathrm{~s}^{-1}\right)$, and at solar metallicity. This star is similar to (though less massive than) that used to model PTF11mnb by Taddia et al. (2018b), and with much less He than the star used to model iPTF15dtg by Taddia et al. (2016b). We then exploded this progenitor star with the hydrodynamical code SNEC (Morozova et al. 2015) with a range of different explosion energies, ${ }^{56} \mathrm{Ni}$ masses, and ${ }^{56} \mathrm{Ni}$ mixing parameters.

The model assumes an opacity floor that scales linearly with metallicity, where $\kappa=0.07 \mathrm{~cm}^{2} \mathrm{~g}^{-1}$ at $Z=1$ and $\kappa=$ $0.01 \mathrm{~cm}^{2} \mathrm{~g}^{-1}$ at $Z=0.02$. We obtained bolometric light curves and their rise times for each model. We defined a first model with $E_{K}=7 \times 10^{51} \mathrm{erg}, M_{\mathrm{ej}}=7.34-1.4=5.9 M_{\odot}$ (the removed $1.4 M_{\odot}$ from the total mass of the star accounts for the compact remnant), ${ }^{56} \mathrm{Ni}$ mixing up to $80 \%$ of the ejecta mass, and a ${ }^{56} \mathrm{Ni}$ mass of $0.3 M_{\odot}$. Then, we kept all of these parameters constant except the ${ }^{56} \mathrm{Ni}$ mass, which we also set to 0.1 and $0.5 M_{\odot}$. Subsequently, we varied the explosion energy with the values 2.0 , $3.0,4.5,7.0,9.5,12,14.5,19.5$, and $24.5 \times 10^{51} \mathrm{erg}$, keeping all of the other parameters unchanged. Finally, we varied the ${ }^{56} \mathrm{Ni}$ mixing from $40 \%$ to $100 \%$.
We plot all of the models and their rise times versus the mentioned parameters in Fig. 24. This shows how these parameters correlate with the rise time. We derived simple scaling relations between these parameters and the rise time - in particular, a linear relation with ${ }^{56} \mathrm{Ni}$ mixing and ${ }^{56} \mathrm{Ni}$ mass, and a power-law scaling with $E_{K} / M_{\mathrm{ej}}$. The best-fit relations are shown in red in the figure. We calibrated these relations using the known rise time of SN 1998bw (15.5d) and the progenitor parameters of SN 1998bw from the work of Chugai (2000; see Fig. 24). SN 1998bw is believed to be fully mixed. These calibrated scaling relations require the rise time of a SN Ic-BL, its $E_{K} / M_{\mathrm{ej}}$, and its ${ }^{56} \mathrm{Ni}$ mass to determine the degree of ${ }^{56} \mathrm{Ni}$ mixing. We used the bolometric rise times reported in Fig. 17 and the $E_{K} / M_{\text {ej }}$ and ${ }^{56} \mathrm{Ni}$ mass parameters from the Arnett fit in Table 4. We did this for all of the radioactively powered $\mathrm{SNe}$ in our sample, obtaining the mixing reported in Table 4 and shown in Fig. 24. All of the SNe are strongly mixed (>87\%) and more than $70 \%$ of these $\mathrm{SNe}$ are fully mixed. We note that $E_{K} / M_{\mathrm{ej}}$ in our models comes from the constraints on the photospheric velocity, so it is independent of the early light-curve shape, from which we estimate the ${ }^{56} \mathrm{Ni}$ mixing with our scaling relations. 

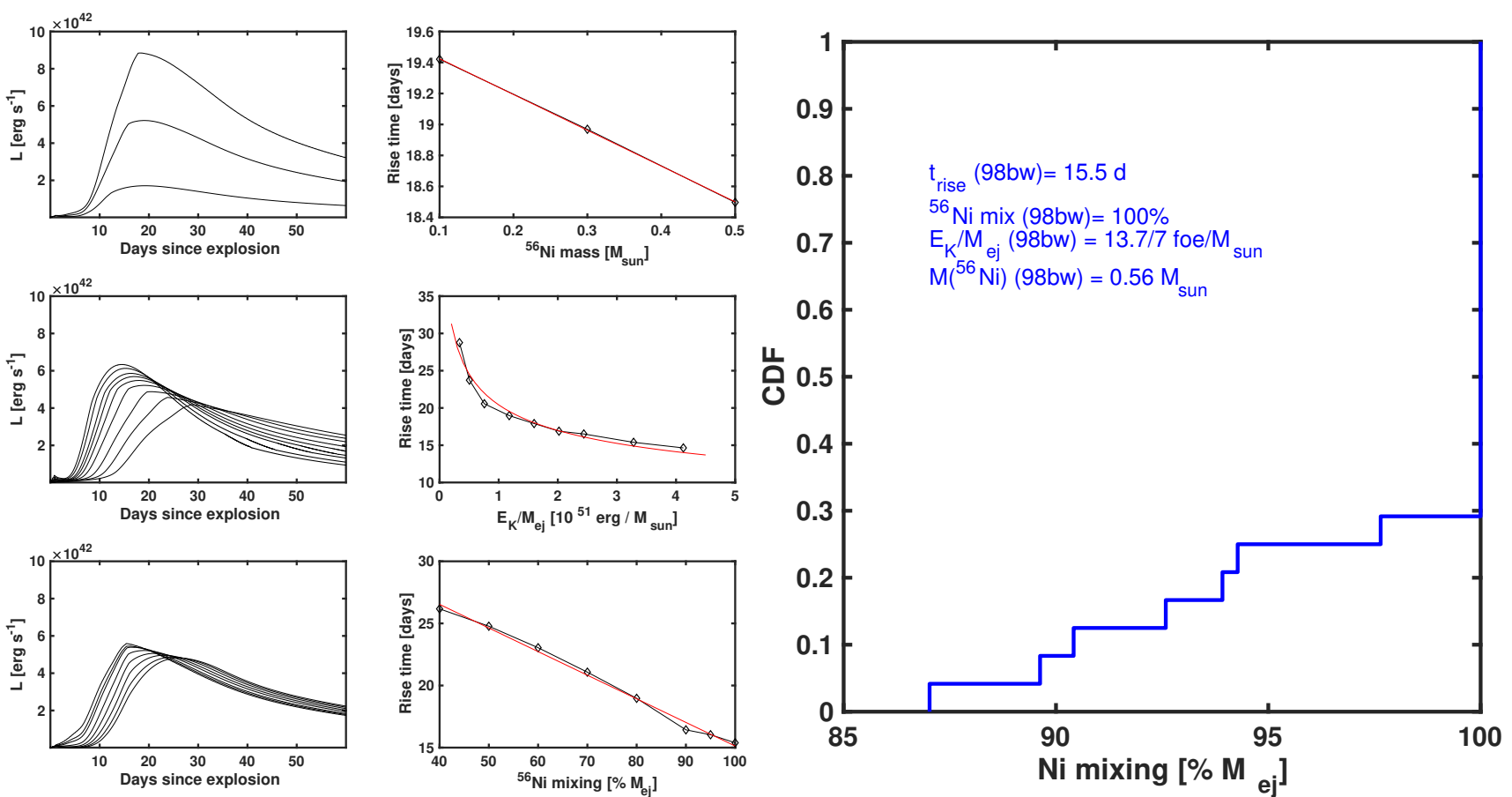

Fig. 24. Left panels: light curves from the hydrodynamical models and their rise times as a function of three explosion and progenitor parameters ( ${ }^{56} \mathrm{Ni}$ mass, $E_{K} / M_{\mathrm{ej}},{ }^{56} \mathrm{Ni}$ mixing). The observed correlations are fit with two linear and one power-law functions. These functions are used to build scaling relations aimed at determining the ${ }^{56} \mathrm{Ni}$ mixing of our SNe, relations that are calibrated against the parameters of SN 1998bw from Chugai (2000). Right panel: ${ }^{56} \mathrm{Ni}$ mixing obtained from the scaling relations determined from the hydrodynamical models and calibrated to SN $1998 \mathrm{bw}$. All of the SNe are strongly (>87\%) mixed.

\section{Discussion}

\subsection{Literature comparison}

First, we emphasize that PTF and iPTF, as compared with the other SN Ic-BL samples, provide a larger, homogeneous, untargeted set with good constraints on the explosion epoch. Furthermore, this work makes use of state-of-the-art host extinction and bolometric corrections, and spectral velocity measurements; moreover, in addition to deriving the classic explosion parameters, it also tests the role of ${ }^{56} \mathrm{Ni}$ mixing.

A number of SNe Ic-BL and GRB-SNe have been presented in the literature. Our PTF+iPTF sample of SNe Ic-BL is mainly composed of SNe not associated with GRBs. We note that we could only determine the explosion parameters for 1 GRB-SN of the 25 events. In the following we do not consider the parameters for iPTF15eov and iPTF16asu since those SNe are not radioactively powered.

We checked for any eventual GRB coincidence in time and space with our SNe using the Fermi archive and the GRB interplanetary network ${ }^{6}$. We did not find GRB associations aside from those already known from the literature of iPTF13bxl (and iPTF14bfu, for which we did not have enough photometry to perform an analysis).

Therefore, we start by comparing the explosion and progenitor parameters of the $24 \mathrm{PTF}+\mathrm{iPTF}$ SNe Ic-BL not associated with a GRB to samples of other similar events, excluding GRBSNe. Cano (2013) presented explosion parameters for $9 \mathrm{SNe}$ Ic-BL not associated with a GRB. Taddia et al. (2015) showed four events, whereas Lyman et al. (2016) included $5 \mathrm{SNe}$ Ic-BL without GRB association. Taddia et al. (2018a) presented 2 additional SNe Ic-BL, namely SN 2009bb and SN 2009ca. Our

\footnotetext{
6 https://heasarc.gsfc.nasa.gov/W3Browse/all/ipngrb.
} html
iPTF sample ( 24 objects) therefore more than doubles the sum of the samples (20) for which explosion parameters have been derived. We also note that Prentice et al. (2016) estimated the ${ }^{56} \mathrm{Ni}$ mass for a collection of $12 \mathrm{SNe}$ Ic-BL not associated with GRBs (and of $10 \mathrm{GRB} \mathrm{SNe}$ ), but they provided only $M_{\mathrm{ej}}^{3} / E_{K}$. Also Drout et al. (2011) presented ${ }^{56} \mathrm{Ni}$ mass values for $5 \mathrm{SNe}$ Ic-BL, and values for $M_{\mathrm{ej}}^{3 / 4} / E_{K}^{1 / 4}$. In Fig. 23, we present the cumulative distribution functions of the main parameters, as compared to those obtained by the other mentioned studies in the literature. In Table 5 we report averages and standard deviations of the different samples.

These works make use of similar assumptions regarding the opacity $\left(\kappa=0.05-0.07 \mathrm{~cm}^{2} \mathrm{~g}^{-1}\right)$ used in the Arnett models. Cano (2013) did not assume $E / M=(3 / 10) v^{2}$ as in the other works, but $E / M=(1 / 2) v^{2}$. The velocities derived for our iPTF SNe are from Fe II and determined via the robust method proposed by Modjaz et al. (2016). In the other works, the velocities are mainly from Si II. The work by Lyman et al. (2016) is that with which the comparison is more direct owing to the most similar assumptions in the modeling.

The ejecta masses turn out to be rather similar among the samples, confirming relatively low values for this parameter. Compared to Lyman et al. (2016) who found $3.3 \pm 2.7 M_{\odot}$, we found $4.0 \pm 2.9 M_{\odot}$. Our sample has a tail of the ejecta-mass probability distribution that extends to larger masses than that of Lyman et al. (2016); the implication of this is discussed in Sect. 8.

In the case of Cano (2013; and Taddia et al. 2015, which has fewer events), the derived kinetic energies are higher than in our sample. The difference is statistically significant $(p=0.037$ through a K-S test). However, we stress that in Cano (2013) the assumption on $E_{K} / M_{\mathrm{ej}}$ relative to the photospheric velocity was 
Table 5. Averages of supernova explosion parameters.

\begin{tabular}{lccc}
\hline \hline Sample (size) & $\begin{array}{c}M_{\mathrm{ej}} \\
\left(M_{\odot}\right)\end{array}$ & $\begin{array}{c}E_{K} \\
\left(10^{51} \mathrm{erg}\right)\end{array}$ & $\begin{array}{c}M\left({ }^{56} \mathrm{Ni}\right) \\
\left(M_{\odot}\right)\end{array}$ \\
\hline Drout et al. 2011 (5) & $\ldots$ & $\ldots$ & $0.570(0.541)$ \\
Cano 2013 (9) & $5.4(3.4)$ & $12.6(8.9)$ & $0.299(0.274)$ \\
Taddia et al. 2015 (4) & $5.4(2.6)$ & $10.7(9.4)$ & $1.110(0.580)$ \\
Lyman et al. 2016(5) & $3.3(2.7)$ & $6.1(6.5)$ & $0.322(0.162)$ \\
Prentice et al. 2016(9) & $\ldots$ & $\ldots$ & $0.151(0.073)$ \\
This work (24) & $3.9(2.8)$ & $7.1(5.7)$ & $0.309(0.164)$ \\
\hline
\end{tabular}

Notes. From Arnett semi-analytic models of SNe Ic-BL (not associated with GRBs) from the literature and this work. Uncertainties in the averages are the standard deviations. We removed the GRB SN iPTF13bxl and the peculiar iPTF15eov and iPTF16asu from our sample when computing the average. Prentice et al. (2016) do not include extinction corrections for some $\mathrm{SNe}$ Ic-BL.

different by a factor of $5 / 3$ compared to the other works, implying higher energy.

The estimated ${ }^{56} \mathrm{Ni}$ masses are similar for all of the samples except Taddia et al. (2015), where the mean value is considerably higher. However, this can be understood in terms of the average redshift of the sample shown by Taddia et al. (2015), which is much higher than in the other samples, and therefore biased toward brighter, more ${ }^{56} \mathrm{Ni}$-rich events (see the left panel in Fig. 23). Moreover, in Taddia et al. (2015), large extinction corrections were applied based on colors; the intrinsic color was taken from a sample of SE SNe that also included SNe Ib and Ic, not only SNe Ic-BL. In Prentice \& Mazzali (2017), which does not provide estimates for $E_{K}$ and $M_{\mathrm{ej}}$ since they do not make use of the SN spectra, some events were not corrected for host extinction, implying a lower average ${ }^{56} \mathrm{Ni}$ mass.

We further investigated the effect of the Malmquist bias on the ${ }^{56} \mathrm{Ni}$-mass distribution for our $\mathrm{SNe}$, by studying its effect on the peak absolute $r$-band magnitude distribution. Following the approach by Richardson et al. (2014), we plot the peak absolute magnitudes versus their respective distance moduli in Fig. 25. We identify the minimum peak magnitude at -17.74 mag in accordance with the faintest objects in our sample. Given the apparent magnitude limit of iPTF (20.5 mag) and considering the extinction, this sets the distance modulus within which we observe the complete peak magnitude distribution to 37.88 mag. The fraction of SNe between -17.74 and $-18.74 \mathrm{mag}$ as well as between -18.74 and -19.74 mag within a distance modulus of $37.88 \mathrm{mag}$ is considered intrinsic, and we reproduce the same fraction at larger distances (distance moduli of 37.88$38.88 \mathrm{mag}$ and $38.88-39.88 \mathrm{mag}$ ) by randomly simulating the magnitude of the missing $\mathrm{SNe}$ in those bins. The distribution of the peak magnitudes after creating these new SNe has an average of $-18.55 \pm 0.63 \mathrm{mag}$ versus the previous $-18.75 \pm 0.66 \mathrm{mag}$ (see Fig. 25). A difference of $0.2 \mathrm{mag}$ in peak luminosity means that the Malmquist-bias corrected ${ }^{56} \mathrm{Ni}$ mass distribution has an average that is $\sim 17 \%$ lower than that of the observed ${ }^{56} \mathrm{Ni}$ mass distribution. Overall, this is a rather small effect.

Our sample is untargeted, so we found SNe in all kinds of star-forming galaxies within a span of host galaxy magnitudes larger than $7 \mathrm{mag}$ in the $g$ band (see Table 1 and Fig. 26). In Fig. 26, we plot the $r$ light-curve properties versus the host galaxy $g$ absolute magnitude; despite the large range of host galaxy luminosity, we do not see clear differences between the populations at low and high host galaxy luminosity.
Finally, we compare the main parameters of our sample to those of 14 GRB-SNe from Cano (2013, see their Table 1). Their average ejecta mass and the kinetic energy are $6.34 \pm 4.40 M_{\odot}$ and $2.49 \pm 1.77 \times 10^{52} \mathrm{erg} \mathrm{s}^{-1}$, the average ${ }^{56} \mathrm{Ni}$ mass $0.40 \pm$ $0.27 M_{\odot}$. The averages from Cano (2013) are higher than those of our sample (especially the kinetic energy), but still compatible within the uncertainties.

\subsection{Implications for progenitor stars and powering mechanism}

SNe Ic-BL and GRB SNe have been suggested to come from massive stars, like Wolf-Rayet (WR) stars, which have initial masses higher than $25-30 M_{\odot}$, typically have fast rotation (Woosley \& Heger 2006), and have low metallicity (e.g., Sanders et al. 2012). From the Arnett models, we derived progenitor and explosion parameters for $27 \mathrm{SNe}$ Ic-BL, assuming these $\mathrm{SNe}$ to be powered by radioactivity. By looking at the top panel of Fig. 21, it is clear that both iPTF15eov and iPTF16asu would require a mass of ${ }^{56} \mathrm{Ni}$ that is more than half of their ejecta masses according to the Arnett model. This suggests that the powering mechanism of these $\mathrm{SNe}$ is not (only) radioactive decay, but rather another mechanism such as the spindown of a magnetar (Kasen \& Bildsten 2010). The other $25 \mathrm{SNe}$ are compatible with radioactive powering of their light curves, and their derived parameters can be used to constrain the nature of their progenitor stars. The ejecta masses are characterized by a majority of events $(64 \%)$ having $M_{\text {ej }}<4.5 M_{\odot}$ and the peak of the asymmetric distribution around $2 M_{\odot}$ (see Fig. 22). A non-negligible fraction (21\%) of the $\mathrm{SNe}$ have ejecta masses above $5.5 M_{\odot}$. This is larger than what was found by Lyman et al. (2016), where the probability of finding $\mathrm{SNe}$ Ic-BL with ejecta masses above $5.5 M_{\odot}$ was almost negligible. Our SNe with ejecta masses higher than $5.5 M_{\odot}$ could be consistent with single massive $\left(M_{\text {ZAMS }}>28 M_{\odot}\right)$ WR-star progenitors, as shown by Lyman et al. (2016). The other SNe exhibit lower ejecta masses, which might rather come from binary stars with lower initial masses $\left(M_{\text {ZAMS }}<28 M_{\odot}\right)$.

An alternative hypothesis to pre-supernova mass loss could be that the observations of lower ejecta masses imply that some of the progenitor-star material fell back onto the forming black hole and did not participate to form the ejecta (Colgate 1971). However, the large amount of ${ }^{56} \mathrm{Ni}$ in SNe Ic-BL and the latetime nebular abundances of core-collapse SNe (Jerkstrand et al. 2018) might disfavor this scenario.

Another possibility is that we inferred low ejecta masses (and also high energies and high ${ }^{56} \mathrm{Ni}$ masses) as a consequence of the assumption related to the symmetry of the ejecta. This is an important caveat to discuss. We assumed spherical symmetry in the modeling of our SN bolometric light curves, since we fit these with an Arnett model. However, we know that SN 1998bw was likely characterized by asphericity, as discussed and suggested by many works (e.g., Maeda et al. 2006; Tanaka et al. 2007; Dessart et al. 2017). This was concluded from the analysis of the early-time and late-time emission of SN 1998bw, which provides different and inconsistent results in terms of derived ejecta masses, explosion energy, and ${ }^{56} \mathrm{Ni}$ masses. When assuming spherical symmetry, the early peak of the light curve of SN 1998bw is compatible with low $\left(\sim 3 M_{\odot}\right)$ ejecta mass, large explosion energy (a few $10^{52} \mathrm{erg}$ ), and $0.5 M_{\odot}$ of ${ }^{56} \mathrm{Ni}$ (Dessart et al. 2017). However, the interpretation of the late-time light curve suggests larger ejecta mass $\left(\sim 10 M_{\odot}\right)$, lower explosion energy $\left(10^{51} \mathrm{erg}\right.$ ), and only $0.1 M_{\odot}$ of ${ }^{56} \mathrm{Ni}$ (Dessart et al. 2017). Maeda et al. (2003) tried to reconcile these conflicting 

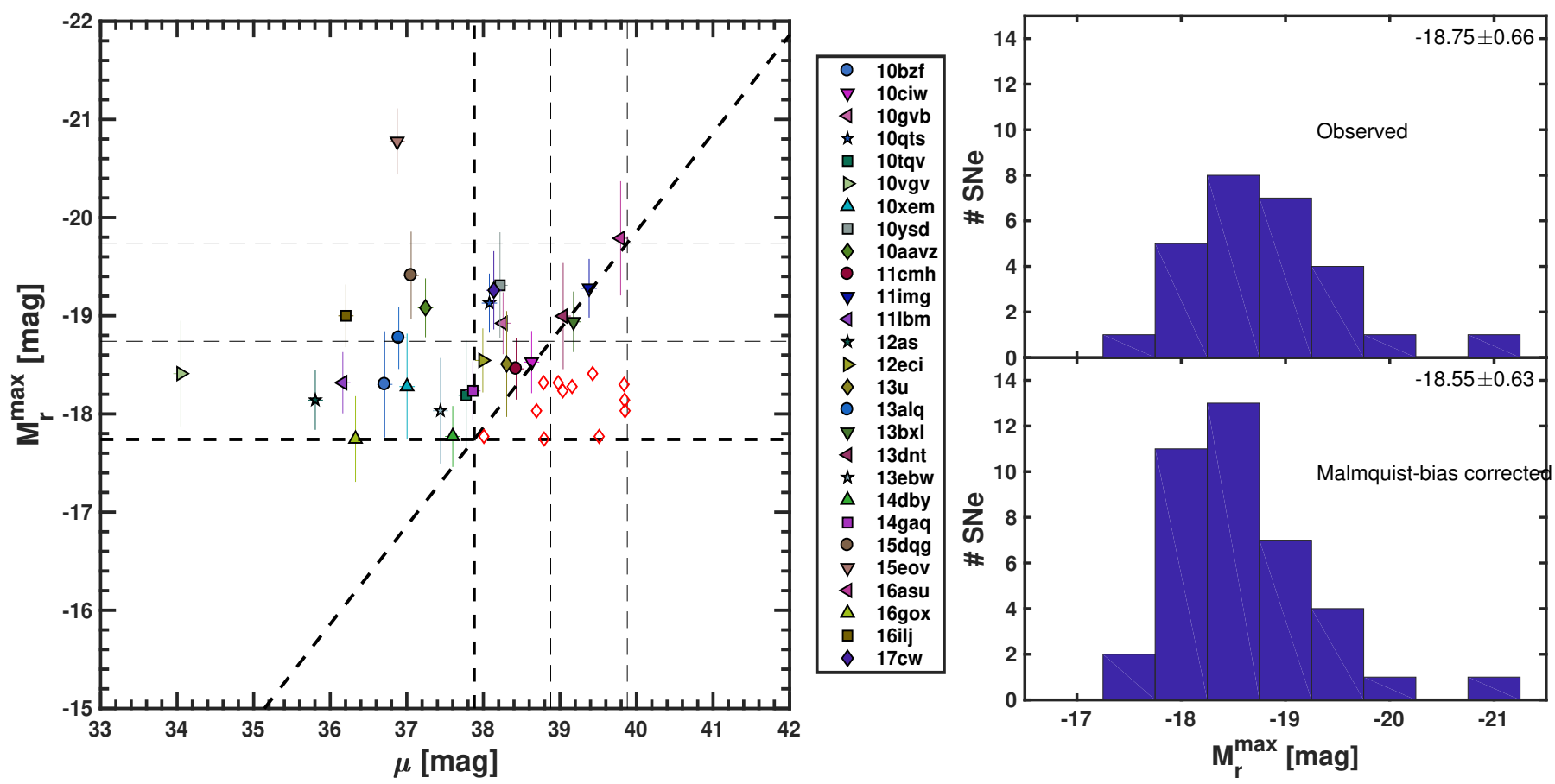

Fig. 25. Left panel: peak absolute magnitude vs. distance modulus. The red empty diamonds are the simulated SNe at large distance moduli (>37.88 mag), where the SN sample is not complete owing to the Malmquist bias (see diagonal dashed line). We followed the method by Richardson et al. (2014, see text in Sect. 8). Right panel: peak magnitude distribution before and after Malmquist-bias correction. It implies a decrease of the ${ }^{56} \mathrm{Ni}$ mass average of about $17 \%$.

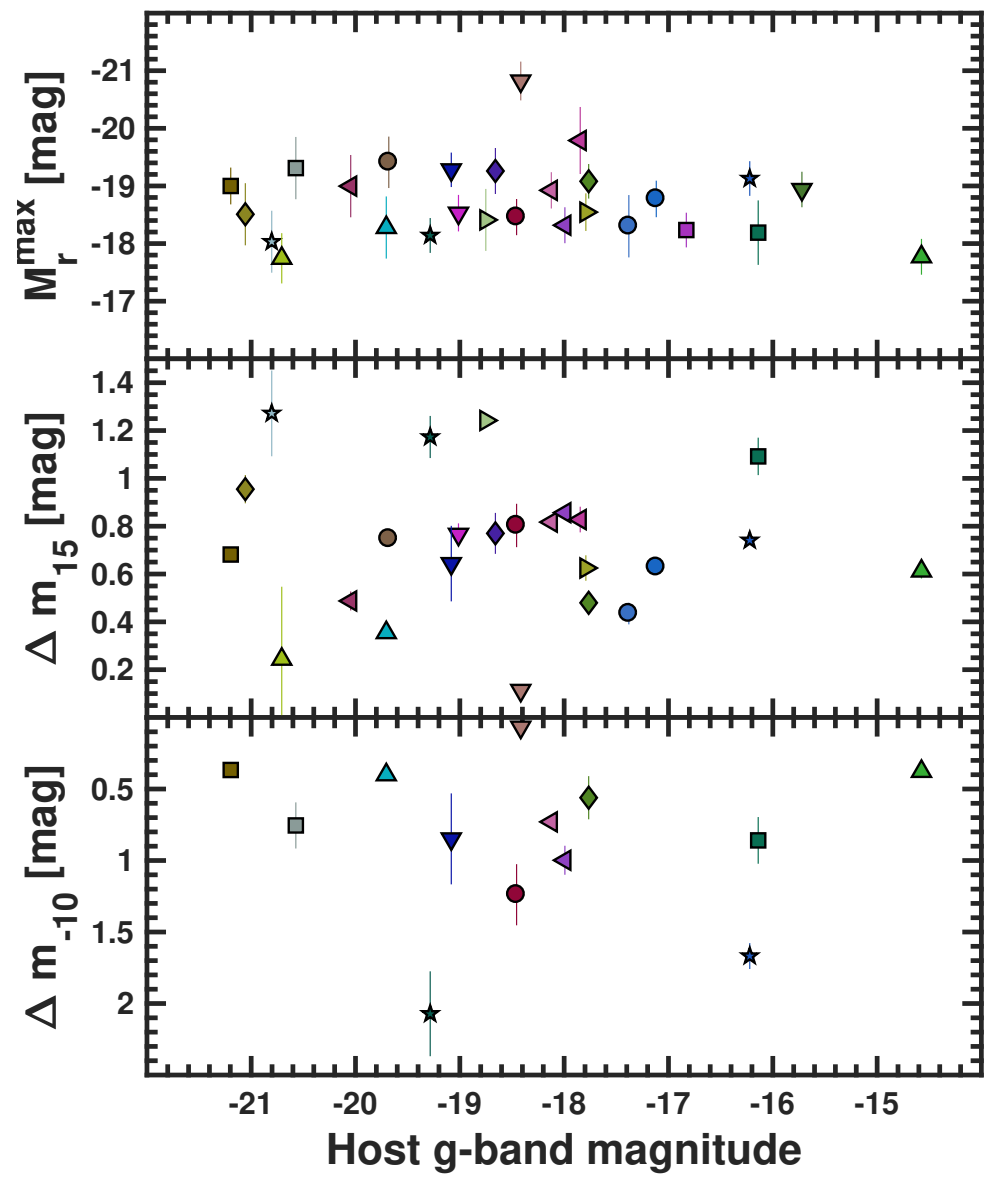

\begin{tabular}{|c|c|}
\hline 0 & 10bzf \\
\hline$\nabla$ & 10ciw \\
\hline$\triangleleft$ & $10 \mathrm{gvb}$ \\
\hline$\star$ & 10 qts \\
\hline 口 & 10 tqv \\
\hline$\triangleright$ & $10 \mathrm{vgv}$ \\
\hline$\Delta$ & 10xem \\
\hline 口 & 10ysd \\
\hline$\diamond$ & 10aavz \\
\hline 0 & $11 \mathrm{cmh}$ \\
\hline$\nabla$ & $11 \mathrm{img}$ \\
\hline$\triangleleft$ & $11 \mathrm{lbm}$ \\
\hline$\star$ & 12as \\
\hline$\triangleright$ & $12 \mathrm{eci}$ \\
\hline$\diamond$ & 13u \\
\hline 0 & 13alq \\
\hline$\nabla$ & 13bxl \\
\hline 4 & 13dnt \\
\hline$\star$ & $13 \mathrm{ebw}$ \\
\hline$\Delta$ & 14dby \\
\hline$\square$ & 14 gaq \\
\hline 0 & $15 \mathrm{dqg}$ \\
\hline$\nabla$ & $15 \mathrm{eov}$ \\
\hline 4 & 16asu \\
\hline & $\begin{array}{l}\text { 16gox } \\
16 \text { ili }\end{array}$ \\
\hline$\Delta$ & $17 \mathrm{cw}$ \\
\hline
\end{tabular}

Fig. 26. Light-curve properties in $r$ vs. the hostgalaxy $g$ absolute magnitude. Over a range of more than 7 mag in the host-galaxy luminosity, we do not see a clear trend in the light-curve properties. 


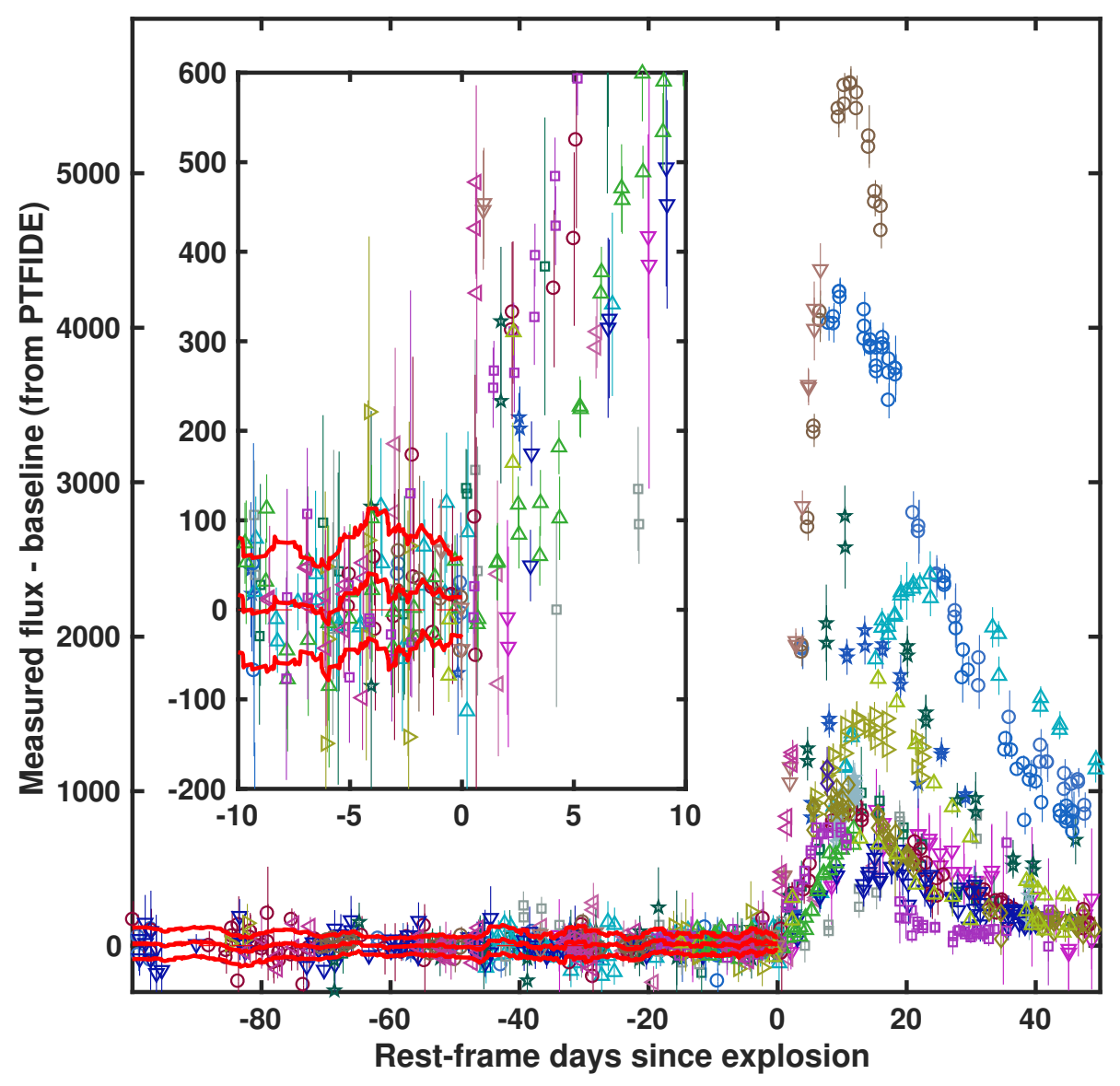

Fig. 27. Observed $r$-band ( $g$-band in the cases of iPTF14gaq, iPTF15dqg, iPTF16asu, and iPTF16gox) fluxes from PTFIDE minus the zero-flux level (baseline) as a function of time since explosion for all of the $\mathrm{SNe}$ with derived explosion epoch and data available a few days before explosion. The inset gives a close-up view of the time range between -10 and $+10 \mathrm{~d}$. No pre-rise excess is observed between -10 and +0 d. Colors and symbols as in Fig. 21. A moving mean over 20 points and its enveloping standard deviations are marked by thick solid red lines, and it is consistent with zero flux. results with a two-zone model in which two different optical depths and Ni masses characterized a slower inner core and a faster envelope. This model still assumes spherical symmetry, which is shown to be inappropriate by Maeda et al. (2006) and Maeda et al. (2008) in further light-curve and late-time spectra investigations of SN 1998bw and other GRB SNe. Dessart et al. (2017) have declared that it is not possible to have a two-zone model with high density in both zones such as that assumed by Maeda et al. (2003).

A solution to the discrepancy between early-time and latetime emission from SN 1998bw could be asymmetric ejecta. Recently, Barnes et al. (2018) used a two-dimensional relativistic hydrodynamical and radiative-transfer model to show that a central engine that triggers long GRBs might also produce a SN Ic-BL. In these models, which account for asymmetry, it is shown that the effect of the viewing angle is small on the early-time light curves. It could be more evident in the spectra; however, in Sect. 5.4 we checked for signs of asymmetry in the spectra of our SNe Ic-BL, but did not find any.

If the ejecta of our SNe Ic-BL are asymmetric, then the progenitor parameters derived from the Arnett model might be substantially different. In particular, as in SN 1998bw, larger ejecta masses would be possible, enlarging the fraction of SNe Ic-BL with ejecta masses consistent with massive single WR stars.

Another potential issue is connected to the explosion epochs that we derived in Sect. 6.1. We assumed that our $\mathrm{SNe}$ can be represented by a stretched version of SN 1998bw. This particular SN did not display any low-luminosity phase between the time of explosion and the light-curve rise, as predicted, for example, by Dessart et al. (2011) for a series of SN Ibc models. In Taddia et al. (2015) we showed that the four SNe Ic-BL in the
SDSS sample did not have such an early dark phase either. However, we tested whether this early dark phase was indeed absent in our iPTF SNe. In Fig. 27, we plot the fluxes from PTFIDE as a function of time since explosion for all of the SNe with estimated explosion epoch, and also with data taken just a few days earlier than the explosion epoch. From the flux obtained from PTFIDE, we subtracted the zero-flux level (baseline) taking the median of the prediscovery fluxes. We then checked whether in the range between -10 and $0 \mathrm{~d}$ before explosion there is an excess in the fluxes, but we found the data to be consistent with zero flux. Therefore, we conclude that our sample was not characterized by $\mathrm{SNe}$ with early light-curve dark plateau phases. This implies that our explosion epoch estimates are reliable along with the explosion parameters derived using those explosion epochs, such as the large ${ }^{56} \mathrm{Ni}$ mixing. This mixing implies that some mechanism in the $\mathrm{SN}$ explosion brings much inner material to the outer parts of the ejecta.

Yet another possibility that recent works have explored is that SNe Ic-BL are powered by magnetars or by the combination of a magnetar and radioactivity (Mazzali et al. 2014; Wang et al. 2017a,b). We have seen that neither iPTF16asu nor iPTF15eov are compatible with solely radioactive power, whereas the rest of the sample could well be. iPTF has also found other SNe Ic that are possibly powered by magnetars, such as iPTF15dtg (Taddia et al. 2016b) and PTF11mnb (Taddia et al. 2018b). However, these SNe, such as iPTF16asu and iPTF15eov, showed very peculiar light curves, clearly distinct from those of normal SNe Ic and SNe Ic-BL. Cano et al. (2016) showed that magnetars cannot be soley responsible for the emission of SNe Ic-BL. But as pointed out by Dessart et al. (2017), the fact that the energetics of SNe Ic-BL are consistent with a magnetar 
source does not necessarily mean magnetars are the actual powering source.

\subsection{Future perspectives: SNe Ic-BL from Zwicky Transient Facility}

With PTF/iPTF, we found 28 SNe Ic-BL observed before peak brightness, with good $r$-band coverage and additional $g B i$ observations, mainly after the peak. All of these SNe had good classification spectra and around three spectra per SN on average, mainly before and around peak.

With the Zwicky Transient Facility (ZTF) we can discover up to 10 times more SNe, including SNe Ic-BL. Only a fraction of these will be classified, as we have limited spectroscopic time at the different facilities we can use within the ZTF collaboration. With the P48 during the ZTF era, what we can do better than iPTF from the observational point of view will be (i) obtain color information before peak, as ZTF will observe both in $g$ and $r$ during the same night, and (ii) provide even tighter constrains on the explosion epoch, given the higher cadence we can reach with a larger field of view. Aside from P48, to improve our observations of SNe Ic-BL, we will need good spectroscopic and photometric follow-up observations until late epochs, using the different facilities to which the ZTF collaboration has access. With such an improved dataset from ZTF, we will be able to build more accurate bolometric light curves and velocity profiles in order to quantify the progenitor properties with higher precision. Late-epoch data will be of importance to study the asymmetry of the ejecta of these $\mathrm{SNe}$ and test alternative powering models.

\section{Summary}

We have presented the optical light curves of the largest existing homogeneous sample of SNe Ic-BL from an untargeted survey. This sample is characterized by good constraints on the SN explosion epochs thanks to the high cadence of PTF and iPTF. The light-curve shapes demonstrate that the decline parameter and rise parameter are correlated and that fast risers are also fast decliners.

The average peak absolute magnitude in the $r$ band is $-18.6 \pm$ $0.5 \mathrm{mag}$. Expansion velocities from the $\mathrm{SN}$ spectra are calculated with the technique from Modjaz et al. (2016), and are consistent with what was obtained for the SNe Ic-BL in their sample.

Arnett models fitted to the bolometric light curves and to the velocity profiles imply the following average explosion parameters: $\left\langle M_{\mathrm{ej}}\right\rangle=3.9 \pm 2.8 M_{\odot},\left\langle E_{K}\right\rangle=(7.1 \pm 5.7) \times 10^{51} \mathrm{erg}$, and $\left\langle M\left({ }^{56} \mathrm{Ni}\right)\right\rangle=0.31 \pm 0.16 M_{\odot}$. These results generally agree with those previously reported in the literature, but our sample has a number of SNe Ic-BL with large $\left(>5.5 M_{\odot}\right)$ ejecta masses that is clearly higher than the numbers found by others (e.g., Lyman et al. 2016).

The implications for the progenitors of SNe Ic-BL are that at least $21 \%$ of these $\mathrm{SNe}$ are compatible with originating in very massive WR stars $\left(M_{\text {ZAMS }}>28 M_{\odot}\right)$. Of the SNe, 64\% might come from close binary systems in which the progenitor was less massive $\left(M_{\text {ZAMS }}<20 M_{\odot}\right)$. However, our simple models do not account for any asymmetry of the $\mathrm{SN}$ ejecta, which has been discussed by others (e.g., Dessart et al. 2017). This might push the actual ejecta masses to higher values for the SNe. Our hydrodynamical models indicate that our $\mathrm{SNe}$ are strongly mixed and that the ${ }^{56} \mathrm{Ni}$ occurs all the way to the outermost layers.

Finally, we note that ZTF will allow us to observe $\sim 10$ times more SNe Ic-BL, will have better color information at early epochs, and tighter constraints on the explosion epoch. Substantial follow-up observations will be necessary to fully exploit these discoveries.

Acknowledgements. The Oskar Klein Centre is funded by the Swedish Research Council. F.T. and J.S. gratefully acknowledge support from the Knut and Alice Wallenberg Foundation. D.A.H. and G.H. are supported by National Science Foundation (NSF) grant AST-1313484. A.V.F. is grateful for financial assistance from NSF grant AST-1211916, the TABASGO Foundation, the Christopher R. Redlich Fund, and the Miller Institute for Basic Research in Science (U.C. Berkeley). A.G.-Y. is supported by the EU via ERC grant No. 725161, the Quantum Universe I-Core program, the ISF, the BSF Transformative program and by a Kimmel award. The intermediate Palomar Transient Factory project is a scientific collaboration among the California Institute of Technology, Los Alamos National Laboratory, the University of Wisconsin, Milwaukee, the Oskar Klein Center, the Weizmann Institute of Science, the TANGO Program of the University System of Taiwan, and the Kavli Institute for the Physics and Mathematics of the Universe. LANL participation in IPTF is supported by the US Department of Energy as a part of the Laboratory Directed Research and Development program. We acknowledge contributions from the full PTF and iPTF collaborations that made it possible to discover and monitor the SE SNe analyzed in this work. This work was supported by the GROWTH project funded by NSF under grant AST-1545949. We thank the staff at the various observatories at which data were collected. The William Herschel Telescope is operated on the island of La Palma by the Isaac Newton Group of Telescopes in the Spanish Observatorio del Roque de los Muchachos of the Instituto de Astrofsica de Canarias. The data presented herein were obtained in part with ALFOSC, which is provided by the Instituto de Astrofisica de Andalucia (IAA) under a joint agreement with the University of Copenhagen and NOTSA. This work is partly based on observations made with DOLoRes@TNG. Our results made use of the Discovery Channel Telescope (DCT) at Lowell Observatory. Lowell is a private, nonprofit institution dedicated to astrophysical research and public appreciation of astronomy, and it operates the DCT in partnership with Boston University, the University of Maryland, the University of Toledo, Northern Arizona University, and Yale University. The upgrade of the DeVeny optical spectrograph has been funded by a generous grant from John and Ginger Giovale. This work makes use of the Las Cumbres Observatory network and the LCO Supernova Key Project. Research at Lick Observatory is partially supported by a generous gift from Google. This research has made use of the NASA/IPAC Extragalactic Database (NED) which is operated by the Jet Propulsion Laboratory, California Institute of Technology, under contract with the National Aeronautics and Space Administration (NASA). Some of the data presented herein were obtained at the W. M. Keck Observatory, which is operated as a scientific partnership among the California Institute of Technology, the University of California, and NASA. The Observatory was made possible by the generous financial support of the W. M. Keck Foundation. The authors wish to recognize and acknowledge the very significant cultural role and reverence that the summit of Maunakea has always had within the indigenous Hawaiian community. We are most fortunate to have the opportunity to conduct observations from this mountain. We thank Mark Sullivan for contributing spectral data obtained under his observing programs. We acknowledge the large number of observers and those responsible for data reduction who helped acquire the spectroscopic database over the years. Among others, these include S. Adams, C. Badenes, S. Ben-Ami, N. Blagorodnova, J. S. Bloom, J. Botyanszki, K. Burdge, Y. Cao, J. Choi, N. Chotard, K. I. Clubb, D. Cook, N. Cucchiara, A. De Cia, A. Drake, G. Duggan, M. Fraser, M. L. Graham, T. Hashimoto, A. Ho, I. Hook, A. Horesh, E. Hsiao, T. Hung, M. Kandrashoff, M. Kasliwal, E. Kirby, I. Kleiser, S. Knezevic, T. Kupfer, K. Maguire, T. Matheson, A. A. Miller, K. P. Mooley, E. Ofek, Y.-C. Pan, D. Polishook, D. Poznanski, V. Ravi, J. Rex, A. Rubin, B. Sesar, I. Shivvers, A. Sternberg, N. Suzuki, D. Tal, C. Theissen, J. van Roestel. P. Vreeswijk, E. Walker, A. Waszczak, D. Xu, O. Yaron, and B. Zackay. We also thank the staff at the observatories where data were obtained. We acknowledge Umaa Rebbapragada and her group for the work with the machine-learning algorithm that allowed iPTF to discover transients. We thank Maryam Modjaz for the productive discussion on the SN classification.

\section{References}

Ahn, C. P., Alexandroff, R., Allende Prieto, C., et al. 2014, ApJS, 211, 17 Arnett, W. D. 1982, ApJ, 253, 785

Barnes, J., Duffell, P. C., Liu, Y., et al. 2018, ApJ, 860, 38

Benn, C., Dee, K., \& Agócs, T. 2008, Proc. SPIE, 7014, 70146X Berger, E., Kulkarni, S. R., \& Chevalier, R. A. 2002, ApJ, 577, L5 Bianco, F. B., Modjaz, M., Hicken, M., et al. 2014, ApJS, 213, 19 Blagorodnova, N., Neill, J. D., Walters, R., et al. 2018, PASP, 130, 035003 Blondin, S., \& Tonry, J. L. 2007, ApJ, 666, 1024 
Burns, C. R., Stritzinger, M., Phillips, M. M., et al. 2011, AJ, 141, 19 Burns, C. R., Stritzinger, M., Phillips, M. M., et al. 2014, ApJ, 789, 32 Cano, Z. 2013, MNRAS, 434, 1098

Cano, Z. 2014, ApJ, 794, 121

Cano, Z., Bersier, D., Guidorzi, C., et al. 2011, MNRAS, 413, 669

Cano, Z., de Ugarte Postigo, A., Pozanenko, A., et al. 2014, A\&A, 568, A19

Cano, Z., de Ugarte Postigo, A., Perley, D., et al. 2015, MNRAS, 452, 1535

Cano, Z., Johansson Andreas, K. G., \& Maeda, K. 2016, MNRAS, 457, 2761

Cenko, S. B., Fox, D. B., Moon, D.-S., et al. 2006, PASP, 118, 1396

Cenko, S. B., Urban, A. L., Perley, D. A., et al. 2015, ApJ, 803, L24

Chilingarian, I. V., \& Zolotukhin, I. Y. 2012, MNRAS, 419, 1727

Chilingarian, I. V., Melchior, A.-L., \& Zolotukhin, I. Y. 2010, MNRAS, 405, 1409

Chugai, N. N. 2000, Astron. Lett., 26, 797

Colgate, S. A. 1971, ApJ, 163, 221

Contardo, G., Leibundgut, B., \& Vacca, W. D. 2000, A\&A, 359, 876

Conti, P. S. 1976, Mem. Soc. R. Sci. Liège, 9, 193

Corsi, A., Ofek, E. O., Frail, D. A., et al. 2011, ApJ, 741, 76

Corsi, A., Ofek, E. O., Gal-Yam, A., et al. 2012, ApJ, 747, L5

Corsi, A., Gal-Yam, A., Kulkarni, S. R., et al. 2016, ApJ, 830, 42

Corsi, A., Cenko, S. B., Kasliwal, M. M., et al. 2017, ApJ, 847, 54

De Cia, A., Gal-Yam, A., Rubin, A., et al. 2018, ApJ, 860, 100

D'Elia, V., Pian, E., Melandri, A., et al. 2015, A\&A, 577, A116

Dessart, L., Hillier, D. J., Livne, E., et al. 2011, MNRAS, 414, 2985

Dessart, L., Hillier, D. J., Yoon, S.-C., Waldman, R., \& Livne, E. 2017, A\&A, 603, A51

Dressler, A., Bigelow, B., Hare, T., et al. 2011, PASP, 123, 288

Drout, M. R., Soderberg, A. M., Gal-Yam, A., et al. 2011, ApJ, 741, 97

Faber, S. M., Phillips, A. C., Kibrick, R. I., et al. 2003, Proc. SPIE, 4841, 1657

Filippenko, A. V. 1982, PASP, 94, 715

Filippenko, A. V. 1997, ARA\&A, 35, 309

Fitzpatrick, E. L. 1999, PASP, 111, 63

Fremling, C., Sollerman, J., Taddia, F., et al. 2016, A\&A, 593, A68

Fremling, C., Sollerman, J., Kasliwal, M. M., et al. 2018, A\&A, 618, A37

Galama, T. J., Vreeswijk, P. M., van Paradijs, J., et al. 1998, Nature, 395, 670

Gal-Yam, A. 2012, Science, 337, 927

Greiner, J., Mazzali, P. A., Kann, D. A., et al. 2015, Nature, 523, 189

Guevel, D., \& Hosseinzadeh, G. 2017, dguevel/PyZOGY: Initial release (Version v0.0.1), Zenodo

Hill, G. J., Nicklas, H. E., MacQueen, P. J., et al. 1998, Proc. SPIE, 3355, 375

Hook, I. M., Jørgensen, I., Allington-Smith, J. R., et al. 2004, PASP, 116, 425

Jerkstrand, A., Ertl, T., Janka, H.-T., et al. 2018, MNRAS, 475, 277

Kasen, D., \& Bildsten, L. 2010, ApJ, 717, 245

Komatsu, E., Dunkley, J., Nolta, M. R., et al. 2009, ApJS, 180, 330

Kulkarni, S. R. 2013, ATel, 4807, 1

Kulkarni, S. R., Frail, D. A., Wieringa, M. H., et al. 1998, Nature, 395, 663

Kumar, B., Singh, A., Srivastav, S., Sahu, D. K., \& Anupama, G. C. 2018, MNRAS, 473, 3776

Lantz, B., Aldering, G., Antilogus, P., et al. 2004, Proc. SPIE, 5249, 146

Law, N. M., Kulkarni, S. R., Dekany, R. G., et al. 2009, PASP, 121, 1395

Li, X., Hjorth, J., \& Wojtak, R. 2014, ApJ, 796, L4

Liu, Y., \& Modjaz, M. 2014, ArXiv e-prints [arXiv:1405 . 1437]

Lunnan, R., Chornock, R., Berger, E., et al. 2018, ApJ, 852, 81

Lyman, J. D., Bersier, D., \& James, P. A. 2014, MNRAS, 437, 3848

Lyman, J. D., Bersier, D., James, P. A., et al. 2016, MNRAS, 457, 328

Maeda, K., Mazzali, P. A., Deng, J., et al. 2003, ApJ, 593, 931
Maeda, K., Mazzali, P. A., \& Nomoto, K. 2006, ApJ, 645, 1331

Maeda, K., Kawabata, K., Mazzali, P. A., et al. 2008, Science, 319, 1220

Masci, F. J., Laher, R. R., Rebbapragada, U. D., et al. 2017, PASP, 129, 014002

Mazzali, P. A., Walker, E. S., Pian, E., et al. 2013, MNRAS, 432, 2463

Mazzali, P. A., McFadyen, A. I., Woosley, S. E., Pian, E., \& Tanaka, M. 2014, MNRAS, 443, 67

Milisavljevic, D., Margutti, R., Parrent, J. T., et al. 2015, ApJ, 799, 51

Miller, J. S., \& Stone, R. P. S. 1993, Lick Observatory Technical Report No. 66 (University of California, Santa Cruz)

Modjaz, M., Liu, Y. Q., Bianco, F. B., \& Graur, O. 2016, ApJ, 832, 108

Morozova, V., Piro, A. L., Renzo, M., et al. 2015, ApJ, 814, 63

Mould, J. R., Huchra, J. P., Freedman, W. L., et al. 2000, ApJ, 529, 786

Ofek, E. O., Laher, R., Law, N., et al. 2012, PASP, 124, 62

Oke, J. B., \& Gunn, J. E. 1982, PASP, 94, 586

Oke, J. B., Cohen, J. G., Carr, M., et al. 1995, PASP, 107, 375

Paxton, B., Bildsten, L., Dotter, A., et al. 2011, ApJS, 192, 3

Phillips, M. M. 1993, ApJ, 413, L105

Phillips, M. M., Simon, J. D., Morrell, N., et al. 2013, ApJ, 779, 38

Pian, E., Tomasella, L., Cappellaro, E., et al. 2017, MNRAS, 466, 1848

Pignata, G., Stritzinger, M., Soderberg, A., et al. 2011, ApJ, 728, 14

Piro, A. L., \& Nakar, E. 2013, ApJ, 769, 67

Prentice, S. J., \& Mazzali, P. A. 2017, MNRAS, 469, 2672

Prentice, S. J., Mazzali, P. A., Pian, E., et al. 2016, MNRAS, 458, 2973

Prentice, S. J., Ashall, C., Mazzali, P. A., et al. 2018, MNRAS, 478, 4162

Quimby, R. M., Kulkarni, S. R., Kasliwal, M. M., et al. 2011, Nature, 474, 487

Quimby, R. M., De Cia, A., Gal-Yam, A., et al. 2018, ApJ, 855, 2

Rahmer, G., Smith, R., Velur, V., et al. 2008, Proc., SPIE, 7014

Rau, A., Kulkarni, S. R., Law, N. M., et al. 2009, PASP, 121, 1334

Richardson, D., Jenkins, III., R. L., Wright, J., \& Maddox, L. 2014, AJ, 147, 118

Sanders, N. E., Soderberg, A. M., Levesque, E. M., et al. 2012, ApJ, 758, 132

Schlafly, E. F., \& Finkbeiner, D. P. 2011, ApJ, 737, 103

Singer, L. P., Cenko, S. B., Kasliwal, M. M., et al. 2013, ApJ, 776, L34

Soderberg, A. M., Chakraborti, S., Pignata, G., et al. 2010, Nature, 463, 513

Stritzinger, M. D., Taddia, F., Burns, C. R., et al. 2018, A\&A, 609, A135

Taddia, F., Sollerman, J., Leloudas, G., et al. 2015, A\&A, 574, A60

Taddia, F., Sollerman, J., Fremling, C., et al. 2016a, A\&A, 588, A5

Taddia, F., Fremling, C., Sollerman, J., et al. 2016b, A\&A, 592, A89

Taddia, F., Stritzinger, M. D., Bersten, M., et al. 2018a, A\&A, 609, A136

Taddia, F., Sollerman, J., Fremling, C., et al. 2018b, A\&A, 609, A106

Tanaka, M., Maeda, K., Mazzali, P. A., \& Nomoto, K. 2007, ApJ, 668, L19

Taubenberger, S., Pastorello, A., Mazzali, P. A., et al. 2006, MNRAS, 371, 1459

Toy, V. L., Cenko, S. B., Silverman, J. M., et al. 2016, ApJ, 818, 79

Valenti, S., Howell, D. A., Stritzinger, M. D., et al. 2016, MNRAS, 459, 3939

Volnova, A. A., Pruzhinskaya, M. V., Pozanenko, A. S., et al. 2017, MNRAS, 467, 3500

Walker, E. S., Mazzali, P. A., Pian, E., et al. 2014, MNRAS, 442, 2768

Wang, L. J., Yu, H., Liu, L. D., et al. 2017a, ApJ, 837, 128

Wang, L. J., Cano, Z., Wang, S. Q., et al. 2017b, ApJ, 851, 54

Whitesides, L., Lunnan, R., Kasliwal, M. M., et al. 2017, ApJ, 851, 107

Woosley, S. E., \& Bloom, J. S. 2006, ARA\&A, 44, 507

Woosley, S. E., \& Heger, A. 2006, ApJ, 637, 914

Yaron, O., \& Gal-Yam, A. 2012, PASP, 124, 668

Yoon, S.-C., Woosley, S. E., \& Langer, N. 2010, ApJ, 725, 940

Zackay, B., Ofek, E. O., \& Gal-Yam, A. 2016, ApJ, 830, 27 


\section{Appendix A: Additional table}

Table A.1. Spectral log and Fe II velocity measurements.

\begin{tabular}{|c|c|c|c|c|c|c|c|c|}
\hline SN & $\operatorname{Icn}\langle N\rangle^{a}$ & JD & $\begin{array}{c}\text { UT Date } \\
\text { [yyyymmdd] }\end{array}$ & Telescope & 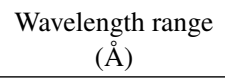 & $\begin{array}{l}\text { Phase [Rest-frame days] } \\
\text { [since } r \text {-band maximum] }\end{array}$ & $\begin{array}{l}\text { Fe II Velocity } \\
\left(\mathrm{km} \mathrm{s}^{-1}\right)\end{array}$ & $\begin{array}{l}T_{\mathrm{BB}} \\
(\mathrm{K}) \\
\end{array}$ \\
\hline 10bzf & 3 & 2455256.5 & 20100301 & GeminiN & $3501-9652$ & -5 & 21660(1501) & $8121(32)$ \\
\hline 10bzf & & 2455262.5 & 20100307 & Keck 1 & $3050-10200$ & 1 & 17664(1541) & 7895(111) \\
\hline 10ciw & $2-3$ & 2455262.5 & 20100307 & Keck 1 & $3342-10239$ & 0 & $21334(8208)$ & $7362(57)$ \\
\hline $10 \mathrm{gvb}$ & 3 & 2455322.9 & 20100506 & Keck 1 & $3500-10000$ & -13 & & $13885(38)$ \\
\hline $10 \mathrm{gvb}$ & & 2455331.5 & 20100515 & Keck 1 & $3300-10180$ & -4 & $15300(1505)$ & $8573(33)$ \\
\hline $10 \mathrm{gvb}$ & & 2455385.5 & 20100708 & Keck 1 & $3120-10040$ & 50 & $7038(1525)$ & 4173(43) \\
\hline $10 \mathrm{qts}$ & $2-3$ & 2455421.5 & 20100813 & P200 & $3505-10100$ & -4 & 19540(1504) & $11934(82)$ \\
\hline 10qts & & 2455423.5 & 20100815 & Lick $3 \mathrm{~m}$ & $3482-10012$ & -2 & $20579(1506)$ & $9069(42)$ \\
\hline $10 \mathrm{qts}$ & & 2455441.5 & 20100902 & P200 & $3440-9850$ & 16 & $20375(1546)$ & $4770(33)$ \\
\hline $10 \mathrm{qts}$ & & 2455444.5 & 20100905 & P200 & $3440-9850$ & 19 & 19595(1985) & $5551(33)$ \\
\hline $10 \mathrm{qts}$ & & 2455448.5 & 20100909 & KPNO 4 m & $3622-8144$ & 23 & $\ldots$ & $5231(33)$ \\
\hline $10 \mathrm{qts}$ & & 2455678.5 & 20110427 & Keck 1 & $3100-11000$ & 253 & $\ldots$ & $\ldots$ \\
\hline 10 tqv & 3 & 2455441.5 & 20100902 & P200 & $3440-9850$ & 4 & & $5208(22)$ \\
\hline 10 tqv & & 2455472.5 & 20101003 & Keck 1 & $3090-10240$ & 35 & $17137(2570)$ & $5080(31)$ \\
\hline $10 \mathrm{tqv}$ & & 2455501.5 & 20101101 & Keck 1 & $3500-7500$ & 64 & 10944(1641) & $\ldots$ \\
\hline $10 \mathrm{vgv}$ & $2-3$ & 2455455.8 & 20100916 & Lick 3 m & $3440-10128$ & -8 & & $9008(20)$ \\
\hline $10 \mathrm{vgv}$ & & 2455466.5 & 20100927 & HET & $4232-10356$ & 3 & $8030(1500)$ & $5471(80)$ \\
\hline $10 \mathrm{vgv}$ & & 2455470.8 & 20101001 & Lick $3 \mathrm{~m}$ & $3440-10700$ & 7 & $\ldots$ & $4971(20)$ \\
\hline $10 \mathrm{vgv}$ & & 2455499.5 & 20101030 & P200 & $3340-9500$ & 36 & $\ldots$ & $4806(31)$ \\
\hline $10 \mathrm{vgv}$ & & 2455536.5 & 20101206 & P200 & 3375-9760 & 73 & $\ldots$ & $\ldots$ \\
\hline 10xem & $2-3$ & 2455479.9 & 20101010 & Lick 3 m & $3500-10000$ & -7 & $14640(1506)$ & 7544(21) \\
\hline $10 x e m$ & & 2455480.5 & 20101011 & KPNO $4 \mathrm{~m}$ & $3840-8400$ & -6 & $10410(5015)$ & $7877(29)$ \\
\hline $10 x e m$ & & 2455501.5 & 20101101 & Keck 1 & $3500-7500$ & 15 & $\ldots$ & $5145(57)$ \\
\hline $10 x e m$ & & 2455593.5 & 20110201 & Keck 1 & $3195-10200$ & 107 & $\ldots$ & $\ldots$ \\
\hline $10 x e m$ & & 2455600.5 & 20110208 & P200 & $3440-9800$ & 114 & $\ldots$ & $\ldots$ \\
\hline $10 y s d$ & 2 & 2455486.5 & 20101017 & P200 & $3450-9900$ & -5 & $14800(1522)$ & 11344(101) \\
\hline 10ysd & & 2455499.5 & 20101030 & WHT & $3500-9498$ & 8 & $\ldots$ & $9877(38)$ \\
\hline $10 \mathrm{ysd}$ & & 2455503.5 & 20101103 & KPNO $4 \mathrm{~m}$ & $3330-8460$ & 12 & $7259(3336)$ & $8278(34)$ \\
\hline 10aavz & 4 & 2455530.5 & 20101130 & WHT & $3100-9348$ & 1 & & $6466(20)$ \\
\hline 10aavz & & 2455536.5 & 20101206 & P200 & $3505-10000$ & 7 & $10630(1506)$ & $5631(21)$ \\
\hline 10aavz & & 2455543.5 & 20101213 & P200 & $3500-9900$ & 14 & $\ldots$ & $6425(55)$ \\
\hline 10aavz & & 2455678.5 & 20110427 & Keck 1 & $3100-10200$ & 149 & $\ldots$ & $\ldots$ \\
\hline $11 \mathrm{cmh}$ & 4 & 2455683.5 & 20110502 & WHT & $3015-11180$ & 1 & $14784(1521)$ & $7422(34)$ \\
\hline $11 \mathrm{img}$ & $3-4$ & 2455772.5 & 20110730 & WHT & $3501-9499$ & 5 & $\ldots$ & $6939(80)$ \\
\hline $11 \mathrm{img}$ & & 2455775.9 & 20110802 & Keck 1 & $3404-10200$ & 9 & $16420(1502)$ & $6395(35)$ \\
\hline $11 \mathrm{lbm}$ & 3 & 2455804.5 & 20110831 & WHT & $3500-9500$ & -1 & $11659(1503)$ & 7931(45) \\
\hline $11 \mathrm{lbm}$ & & 2455825.5 & 20110921 & P200 & $3360-9600$ & 20 & $7815(1560)$ & $6320(26)$ \\
\hline $12 \mathrm{as}$ & 3 & 2455929.0 & 20120102 & APO & $3316-9864$ & -5 & $14950(1516)$ & $7239(75)$ \\
\hline 12as & & 2455956.5 & 20120130 & P200 & $3500-10200$ & 23 & $6995(1688)$ & $7879(42)$ \\
\hline 12as & & 2456044.8 & 20120427 & Keck 1 & 3702-9995 & 111 & & $\ldots$ \\
\hline 12eci & 3 & 2456060.8 & 20120517 & Keck 1 & $3424-10100$ & -7 & $10560(1516)$ & $9005(28)$ \\
\hline $13 \mathrm{u}$ & $\leq 4$ & 2456341.5 & 20130218 & P200 & $3210-10100$ & 11 & 18249(1504) & $5067(25)$ \\
\hline 13alq & 3 & 2456395.5 & 20130413 & P200 & $3600-9900$ & -3 & $21530(1503)$ & $8492(41)$ \\
\hline 13alq & & 2456414.5 & 20130502 & P200 & $3840-10450$ & 16 & $13745(1507)$ & $5932(21)$ \\
\hline 13alq & & 2456414.7 & 20130502 & TNG & $3220-7989$ & 16 & $13745(1507)$ & $5403(22)$ \\
\hline 13alq & & 2456452.8 & 20130609 & Keck 1 & $3080-10284$ & 55 & $\ldots$ & $6139(23)$ \\
\hline $13 \mathrm{bxl}$ & 3 & 2456476.5 & 20130703 & MagellanBaade & $3900-9500$ & 12 & $\ldots$ & $12700(61)$ \\
\hline $13 \mathrm{bxl}$ & & 2456476.7 & 20130703 & P200 & $3700-10000$ & -11 & $\ldots$ & $12362(63)$ \\
\hline $13 \mathrm{bxl}$ & & 2456476.8 & 20130703 & UH88 & 3301-9701 & -11 & $\ldots$ & 7993(26) \\
\hline $13 \mathrm{bxl}$ & & 2456477.7 & 20130704 & $\mathrm{P} 200$ & $3700-10000$ & -10 & $\ldots$ & $15750(137)$ \\
\hline $13 \mathrm{bxl}$ & & 2456478.5 & 20130705 & P200 & $3400-8933$ & -10 & $27780(1532)$ & $14062(112)$ \\
\hline 13bxl & & 2456481.7 & 20130708 & P200 & $4450-9625$ & -6 & $23340(1501)$ & $12486(67)$ \\
\hline $13 \mathrm{bxl}$ & & 2456484.5 & 20130711 & Keck 2 & 4905-10131 & -4 & $20020(1502)$ & $17169(213)$ \\
\hline $13 \mathrm{bxl}$ & & 2456486.5 & 20130713 & Keck 2 & $3700-10840$ & -2 & (1) & 7096(63) \\
\hline $13 \mathrm{bxl}$ & & 2456489.5 & 20130716 & P200 & $4425-9640$ & 1 & $14754(1508)$ & 7997(36) \\
\hline 13bxl & & 2456506.5 & 20130802 & Keck 2 & $3066-10259$ & 18 & $6605(1518)$ & $6344(27)$ \\
\hline $13 \mathrm{bxl}$ & & 2456508.8 & 20130804 & Keck 1 & $3066-10259$ & 21 & $\ldots$ & $6344(27)$ \\
\hline
\end{tabular}

Notes. The double horizontal line separates the spectra for which the phase is known, which were therefore used to measure the Fe II velocity, from those for which it is not. ${ }^{(a)}$ Number of absorption features in the spectra before and around maximum brightness, following the classification method by Prentice \& Mazzali (2017). 
Table A.1. continued.

\begin{tabular}{|c|c|c|c|c|c|c|c|c|}
\hline SN & $\operatorname{Icn}\langle N\rangle^{a}$ & JD & $\begin{array}{c}\text { UT Date } \\
\text { [yyyymmdd] }\end{array}$ & Telescope & 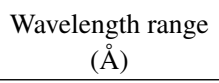 & $\begin{array}{l}\text { Phase [Rest-frame days] } \\
\text { [since } r \text {-band maximum] }\end{array}$ & $\begin{array}{l}\text { Fe II Velocity } \\
\left(\mathrm{km} \mathrm{s}^{-1}\right)\end{array}$ & $\begin{array}{l}T_{\mathrm{BB}} \\
(\mathrm{K}) \\
\end{array}$ \\
\hline $13 \mathrm{dnt}$ & 4 & 2456569.7 & 20131004 & P200 & $3200-10200$ & 4 & $19040(1502)$ & $5575(13)$ \\
\hline $13 \mathrm{ebw}$ & 2 & 2456622.5 & 20131126 & P200 & $3400-10580$ & -8 & & $6148(11)$ \\
\hline $13 \mathrm{ebw}$ & & 2456625.5 & 20131129 & Keck 2 & 4905-10131 & -5 & $\ldots$ & 7903(45) \\
\hline $13 \mathrm{ebw}$ & & 2456630.1 & 20131203 & Keck 1 & $3200-10265$ & -0 & $26584(1505)$ & $5841(15)$ \\
\hline $13 \mathrm{ebw}$ & & 2456631.0 & 20131204 & Keck 1 & $3017-10237$ & 1 & $22004(1505)$ & $5711(21)$ \\
\hline $13 \mathrm{ebw}$ & & 2456658.5 & 20140101 & Keck 2 & 4909-10140 & 28 & $\ldots$ & $5855(18)$ \\
\hline 14dby & 4 & 2456837.9 & 20140629 & Keck 1 & $3057-10288$ & -12 & $16085(1506)$ & $8115(24)$ \\
\hline $14 \mathrm{dby}$ & & 2456899.8 & 20140830 & Keck 1 & 3099-10290 & 49 & $6248(1516)$ & $5272(22)$ \\
\hline $14 \mathrm{dby}$ & & 2456924.7 & 20140924 & Keck 2 & $4600-9599$ & 74 & $\ldots$ & $\ldots$ \\
\hline $14 \mathrm{dby}$ & & 2457045.1 & 20150122 & Keck 1 & $3069-10287$ & 195 & $\ldots$ & $\ldots$ \\
\hline $14 \mathrm{gaq}$ & $2-4$ & 2456924.9 & 20140924 & Keck 2 & $4600-9600$ & -5 & & 9992(23) \\
\hline $14 \mathrm{gaq}$ & & 2456931.5 & 20141001 & P200 & $3000-10239$ & 1 & $24034(1512)$ & $7881(27)$ \\
\hline $14 \mathrm{gaq}$ & & 2456955.7 & 20141025 & Keck 2 & $5000-9998$ & 25 & $\ldots$ & $5491(16)$ \\
\hline $15 \mathrm{dqg}$ & $2-\leq 5$ & 2457333.8 & 20151107 & Keck 2 & $4500-9601$ & -8 & 19935(1519) & 10364(24) \\
\hline $15 \mathrm{dqg}$ & & 2457362.5 & 20151206 & Keck 1 & $3076-10231$ & 20 & $9265(1505)$ & $5527(20)$ \\
\hline $15 \mathrm{dqg}$ & & 2457369.3 & 20151212 & TNG & $3381-10436$ & 27 & $8515(1502)$ & $6029(39)$ \\
\hline $15 \mathrm{eov}$ & $2-4$ & 2457362.5 & 20151206 & Keck 1 & $3069-10233$ & -24 & $20625(1503)$ & 23481(104) \\
\hline $15 \mathrm{eov}$ & & 2457365.0 & 20151208 & FTN & $3249-10000$ & -22 & $20635(1507)$ & 21563(180) \\
\hline 15 eov & & 2457365.9 & 20151209 & FTN & $3201-10000$ & -21 & $20565(1508)$ & $18815(143)$ \\
\hline 15 eov & & 2457367.5 & 20151211 & P200 & 3091-10199 & -19 & $20685(1505)$ & 19400(108) \\
\hline $15 \mathrm{eov}$ & & 2457368.9 & 20151212 & FTN & $3200-10000$ & -18 & $20605(1507)$ & $22267(215)$ \\
\hline $15 \mathrm{eov}$ & & 2457370.0 & 20151213 & FTN & $3200-10001$ & -17 & $20545(1505)$ & $23209(207)$ \\
\hline 15 eov & & 2457371.1 & 20151214 & FTS & $3350-10000$ & -16 & $20685(1505)$ & 29823(681) \\
\hline $15 \mathrm{eov}$ & & 2457374.9 & 20151218 & FTN & $3221-10001$ & -12 & $20625(1505)$ & 19727(192) \\
\hline $15 \mathrm{eov}$ & & 2457384.8 & 20151228 & FTN & $4000-9300$ & -2 & & $31875(3372)$ \\
\hline 15 eov & & 2457392.9 & 20160105 & FTN & 3300-9999 & 6 & 19030(1507) & $9819(29)$ \\
\hline 15 eov & & 2457395.5 & 20160108 & Keck 2 & $4501-9641$ & 9 & $19060(1506)$ & $9549(18)$ \\
\hline 15 eov & & 2457408.9 & 20160121 & FTN & $4000-10000$ & 22 & $17445(1506)$ & $7147(38)$ \\
\hline $15 \mathrm{eov}$ & & 2457416.9 & 20160129 & FTN & $3500-10000$ & 30 & $17872(1505)$ & $6292(23)$ \\
\hline $15 \mathrm{eov}$ & & 2457424.8 & 20160206 & FTN & $3300-10001$ & 38 & $17812(1506)$ & $6543(21)$ \\
\hline $15 \mathrm{eov}$ & & 2457432.8 & 20160214 & FTN & $3800-10000$ & 46 & $17048(1504)$ & $6113(27)$ \\
\hline $15 \mathrm{eov}$ & & 2457436.7 & 20160218 & FTN & $5000-9300$ & 50 & & $6438(81)$ \\
\hline $15 \mathrm{eov}$ & & 2457452.8 & 20160305 & FTN & 3999-10001 & 66 & $17150(1503)$ & $\ldots$ \\
\hline 16asu & $\leq 3$ & 2457522.5 & 20160514 & P200 & $3101-9199$ & -3 & $\ldots$ & $19253(360)$ \\
\hline 16asu & & 2457524.6 & 20160516 & NOT & $3478-9662$ & -1 & $\ldots$ & $17776(370)$ \\
\hline 16asu & & 2457533.5 & 20160524 & TNG & $3315-10330$ & 8 & $27590(1546)$ & $8287(42)$ \\
\hline 16asu & & 2457535.5 & 20160527 & P200 & $3600-10237$ & 10 & 27414(1924) & $8271(52)$ \\
\hline 16asu & & 2457543.9 & 20160604 & Keck 2 & $4550-9649$ & 18 & $24835(1504)$ & $6560(24)$ \\
\hline 16asu & & 2457546.9 & 20160607 & Keck 1 & $3072-10285$ & 21 & $22415(1508)$ & $6343(18)$ \\
\hline 16asu & & 2457549.5 & 20160610 & Keck 1 & $3101-10290$ & 24 & 19882(1513) & $6221(20)$ \\
\hline 16gox & $\leq 3$ & 2457660.5 & 20160929 & P60 & $3807-10456$ & 13 & $\ldots$ & $4694(111)$ \\
\hline 16gox & & 2457662.5 & 20161001 & P200 & 3100-10236 & 15 & $\ldots$ & $4693(21)$ \\
\hline 16 gox & & 2457667.5 & 20161006 & P200 & $3101-10236$ & 20 & $\ldots$ & $5735(16)$ \\
\hline 16 gox & & 2457676.6 & 20161015 & NOT & $3702-9711$ & 29 & $17572(1820)$ & $5039(34)$ \\
\hline 16 ilj & 3 & 2457717.5 & 20161125 & P60 & $3807-10456$ & -4 & & $6352(132)$ \\
\hline $16 \mathrm{ilj}$ & & 2457721.5 & 20161128 & NOT & $3463-9715$ & -0 & 19154(1549) & $6406(62)$ \\
\hline $16 \mathrm{ilj}$ & & 2457722.5 & 20161130 & $\mathrm{P} 60$ & $3807-9187$ & 1 & $\ldots$ & $4859(179)$ \\
\hline $17 \mathrm{cw}$ & $\leq 3$ & 2457760.5 & 20170107 & P200 & $3088-10261$ & -8 & $\ldots$ & $14029(83)$ \\
\hline $17 \mathrm{cw}$ & & 2457785.9 & 20170201 & DCT & $3536-7996$ & 18 & $20595(1528)$ & $5076(34)$ \\
\hline $17 \mathrm{cw}$ & & 2457811.9 & 20170227 & Keck 1 & $3068-10266$ & 44 & $11028(1563)$ & $6104(28)$ \\
\hline 09sk & & 2455008.8 & 20090626 & Keck 1 & $3027-9833$ & & & \\
\hline $10 \mathrm{cs}$ & & 2455205.5 & 20100109 & P200 & $3242-10224$ & & & \\
\hline $10 \mathrm{cs}$ & & 2455262.5 & 20100307 & Keck 1 & $3342-10264$ & & & \\
\hline 12grr & & 2456129.5 & 20120721 & P200 & $3200-10500$ & & & \\
\hline $12 \mathrm{grr}$ & & 2456135.5 & 20120727 & P200 & $3300-10300$ & & & \\
\hline $14 \mathrm{bfu}$ & & 2456816.1 & 20140607 & Keck 2 & $4550-9599$ & & & \\
\hline $14 \mathrm{bfu}$ & & 2456833.0 & 20140624 & Keck 1 & 3116-10279 & & & \\
\hline $14 \mathrm{bfu}$ & & 2456838.1 & 20140629 & Keck 1 & $3234-10289$ & & & \\
\hline 15dld & & 2457333.8 & 20151107 & Keck 2 & $4500-9601$ & & & \\
\hline 15dld & & 2457399.7 & 20160112 & Keck 1 & $3064-10257$ & & & \\
\hline 16coi & & 2457626.5 & 20160826 & P60 & $3807-9187$ & & & \\
\hline $16 \mathrm{coi}$ & & 2457656.6 & 20160925 & NOT & $3407-9647$ & & & \\
\hline $17 \mathrm{axg}$ & & 2457809.5 & 20170225 & P200 & $3500-10924$ & & & \\
\hline $17 \mathrm{axg}$ & & 2457809.9 & 20170225 & P60 & $3807-10456$ & & & \\
\hline
\end{tabular}




\section{Appendix B: Spectral sequences}
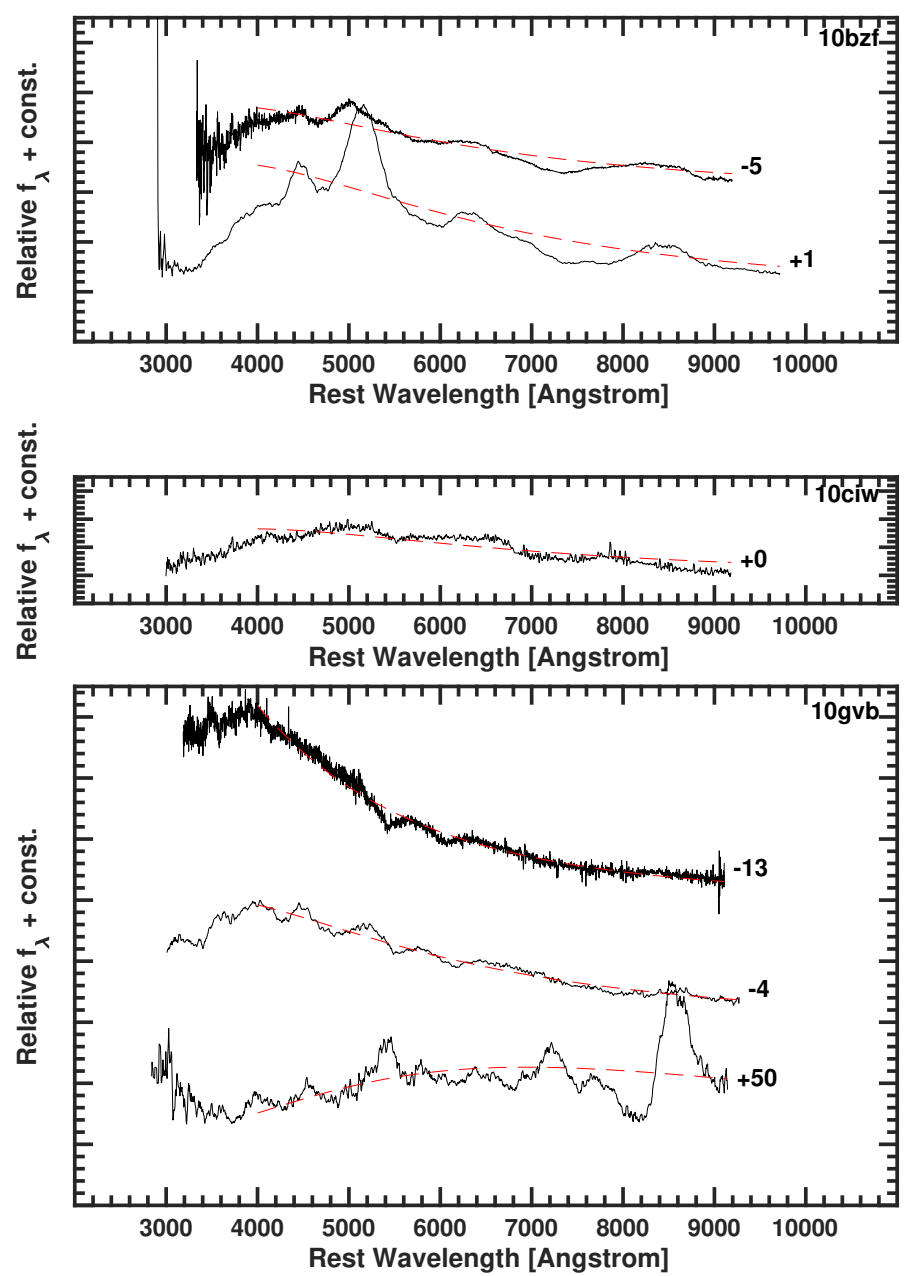

Fig. B.1. Spectral sequences of PTF10bzf, PTF10ciw, and PTF10gvb. The reported phases next to each spectrum are in rest-frame days since $r$-band maximum, and the dashed red lines are black-body fits. This format is followed for Figs. B.2-B.11.
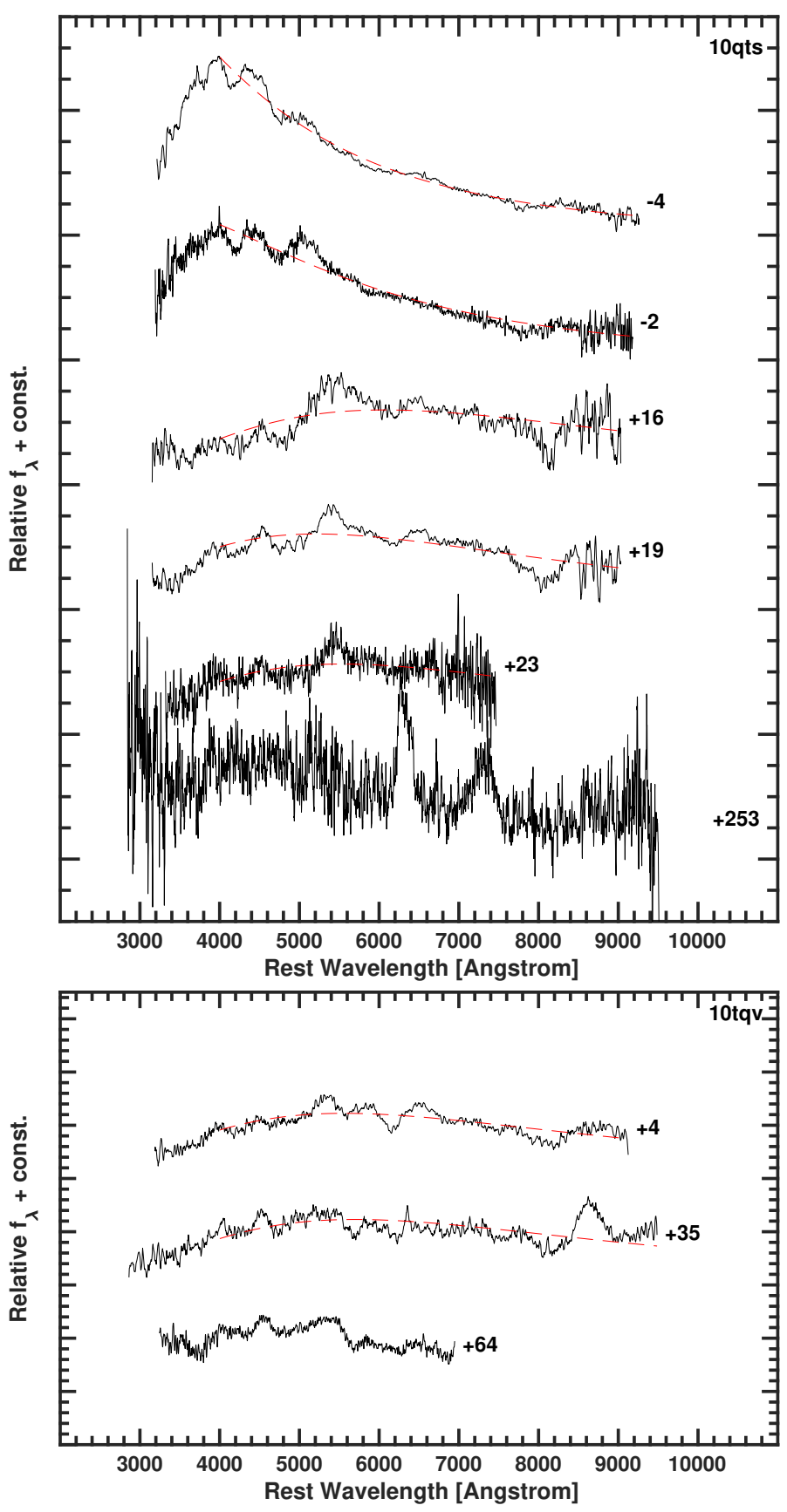

Fig. B.2. Spectral sequences of PTF10qts and PTF10tqv. 

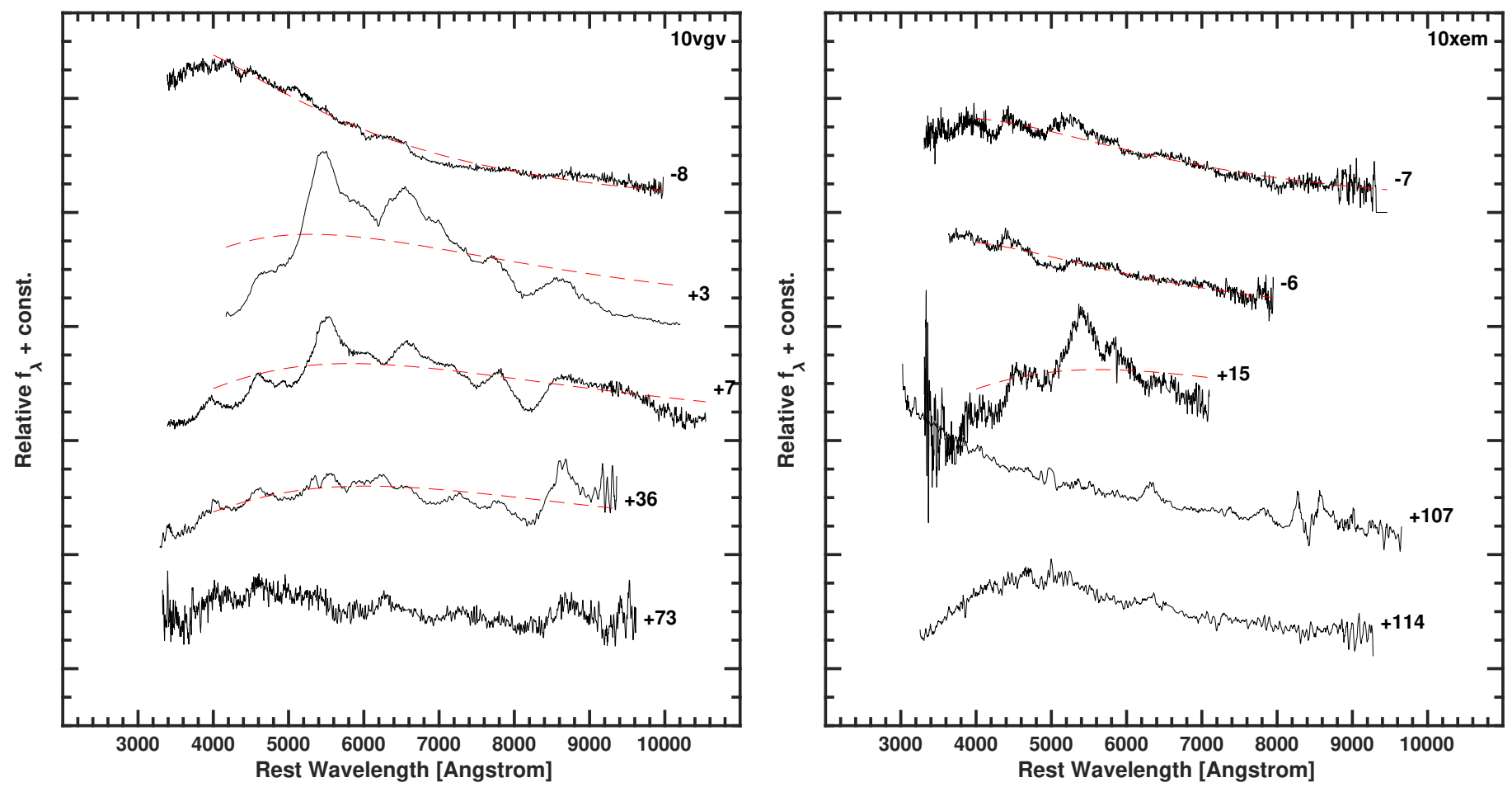

Fig. B.3. Spectral sequence of PTF10vgv.
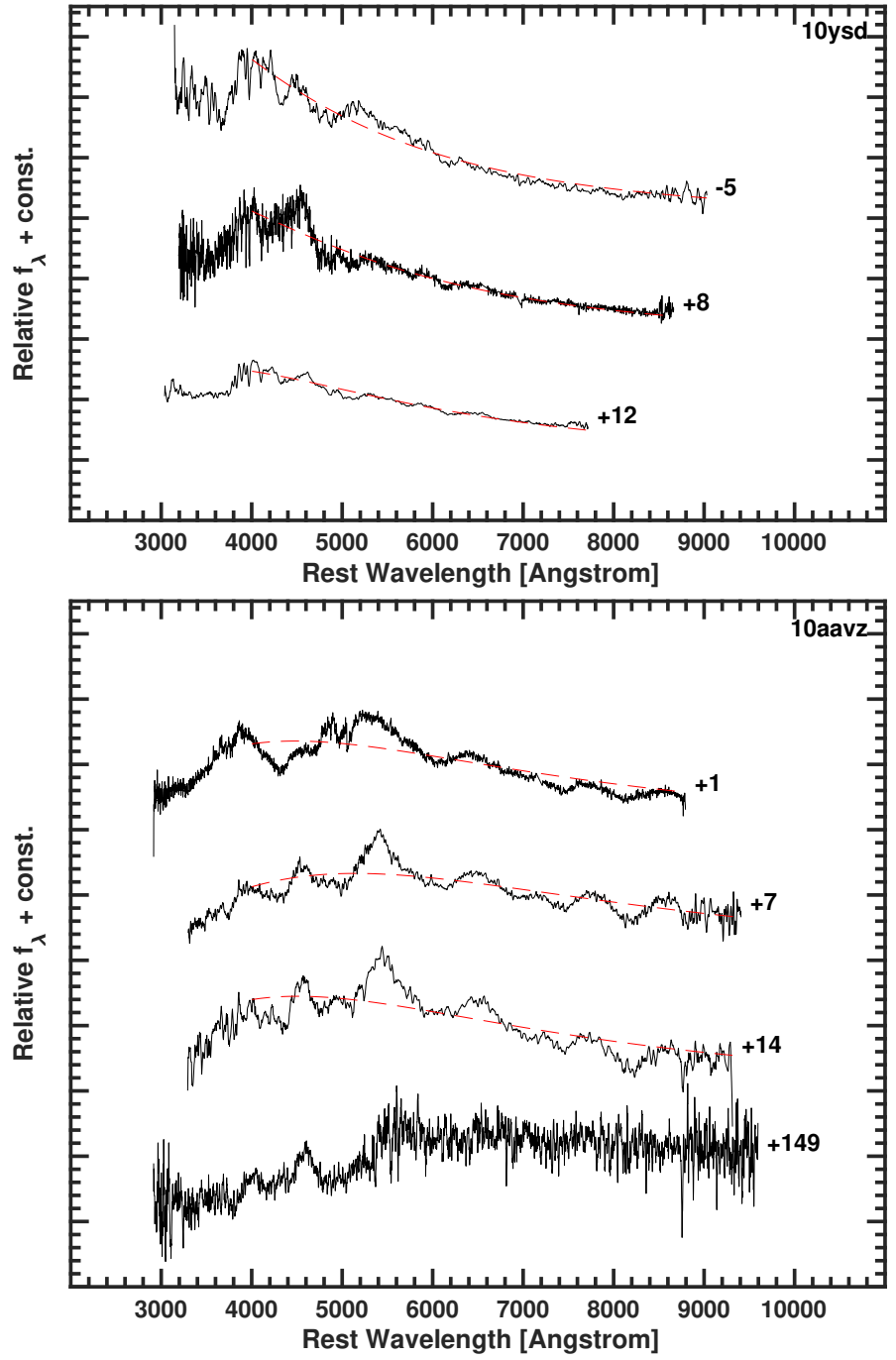

Fig. B.4. Spectral sequences of PTF10xem, PTF10ysd, and PTF10aavz. 

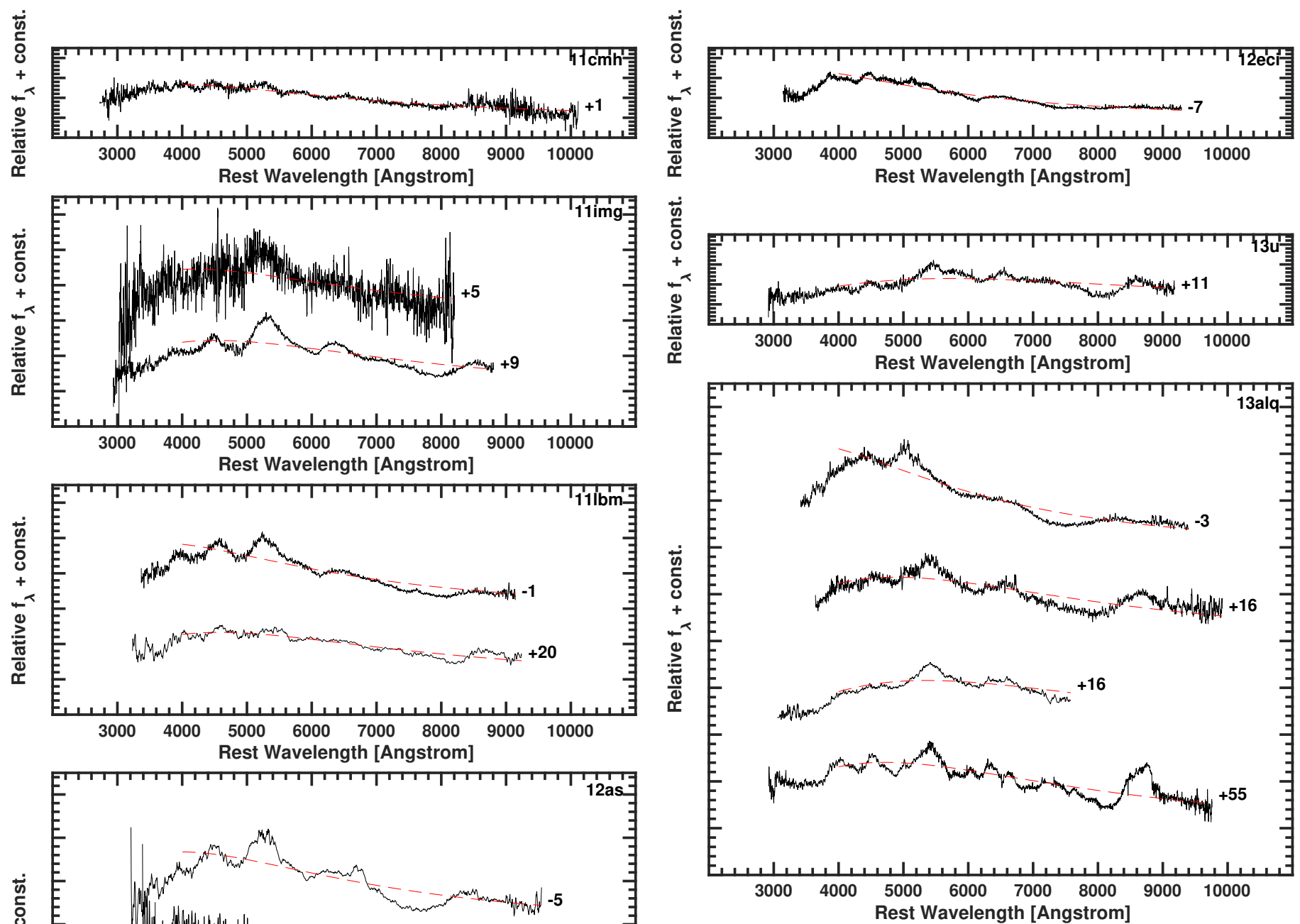

Fig. B.6. Spectral sequences of PTF12eci, iPTF13u, and iPTF13alq.

Fig. B.5. Spectral sequences of PTF11 cmh, PTF11img, PTF11lbm, and PTF12as. 

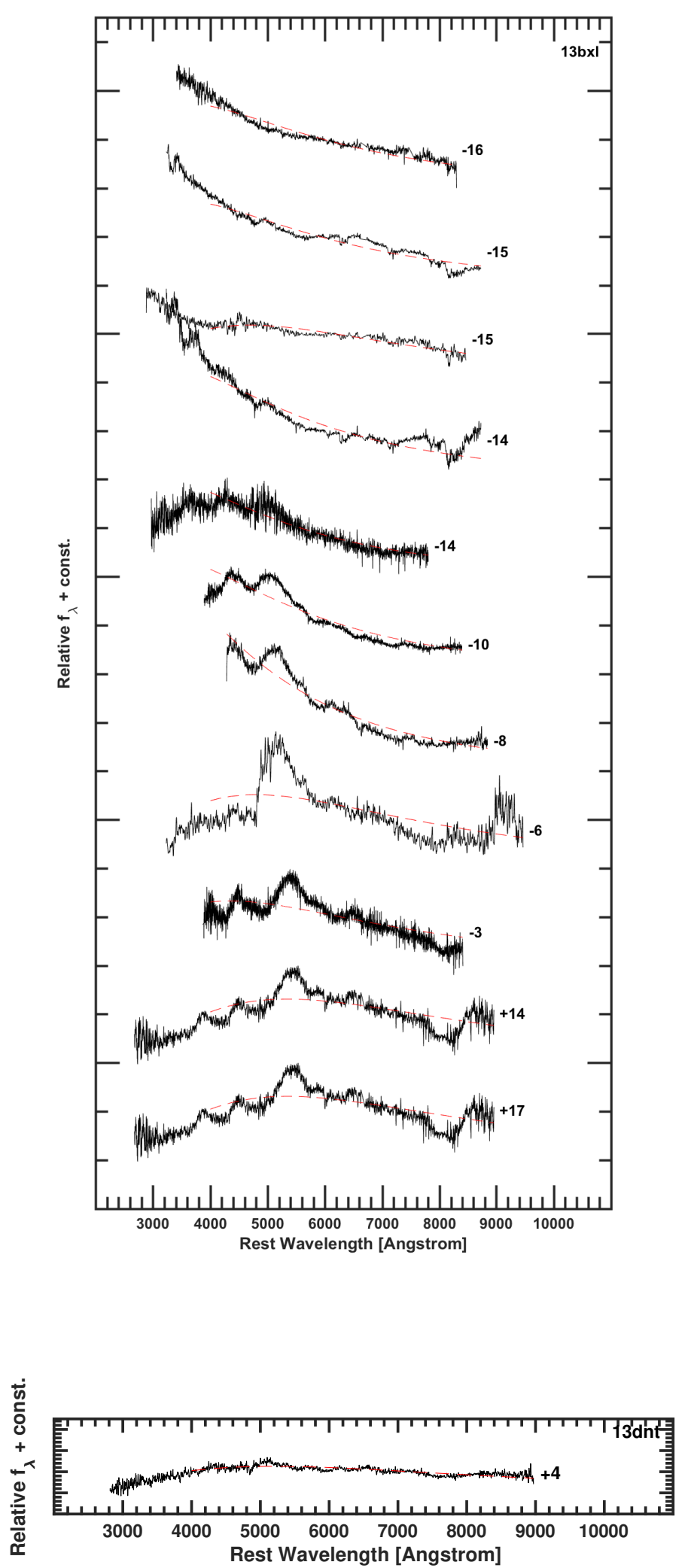

Fig. B.7. Spectral sequences of iPTF13bxl and iPTF13dnt.
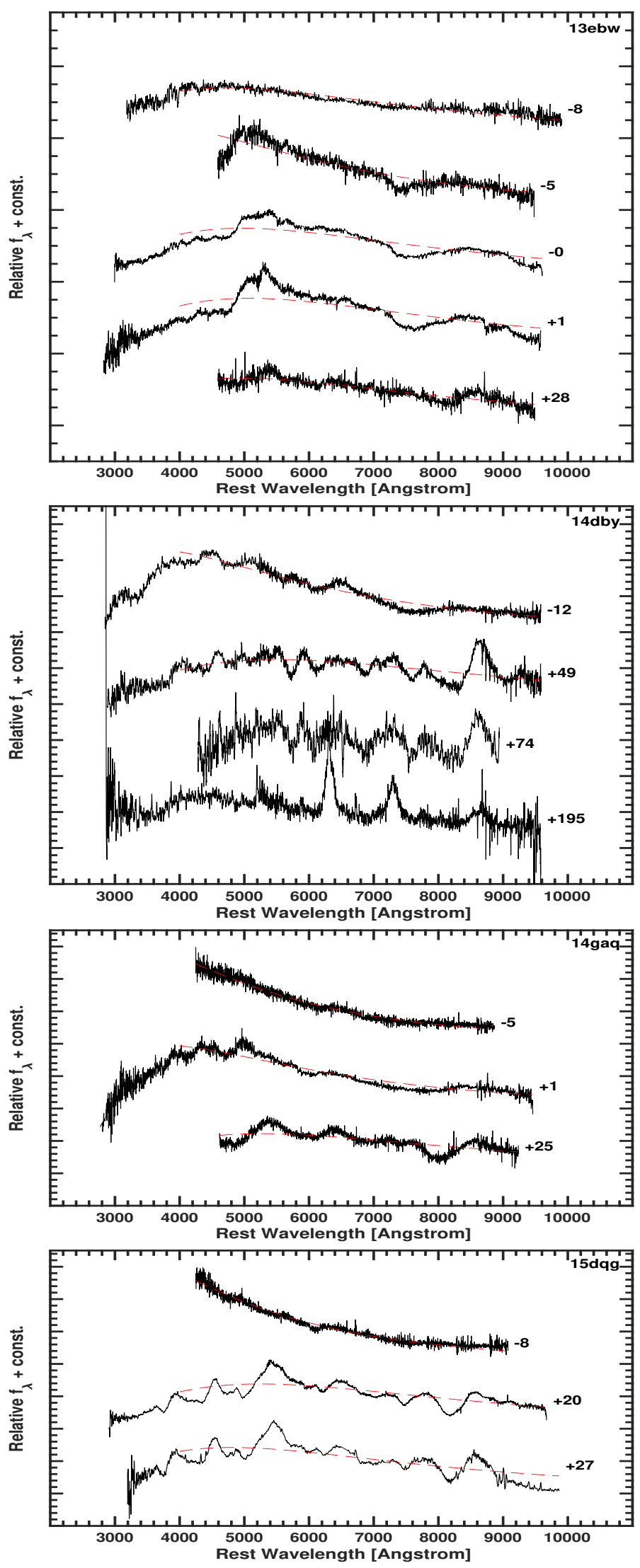

Fig. B.8. Spectral sequences of iPTF13ebw, iPTF14dby, iPTF14gaq, and iPTF15dqg. 

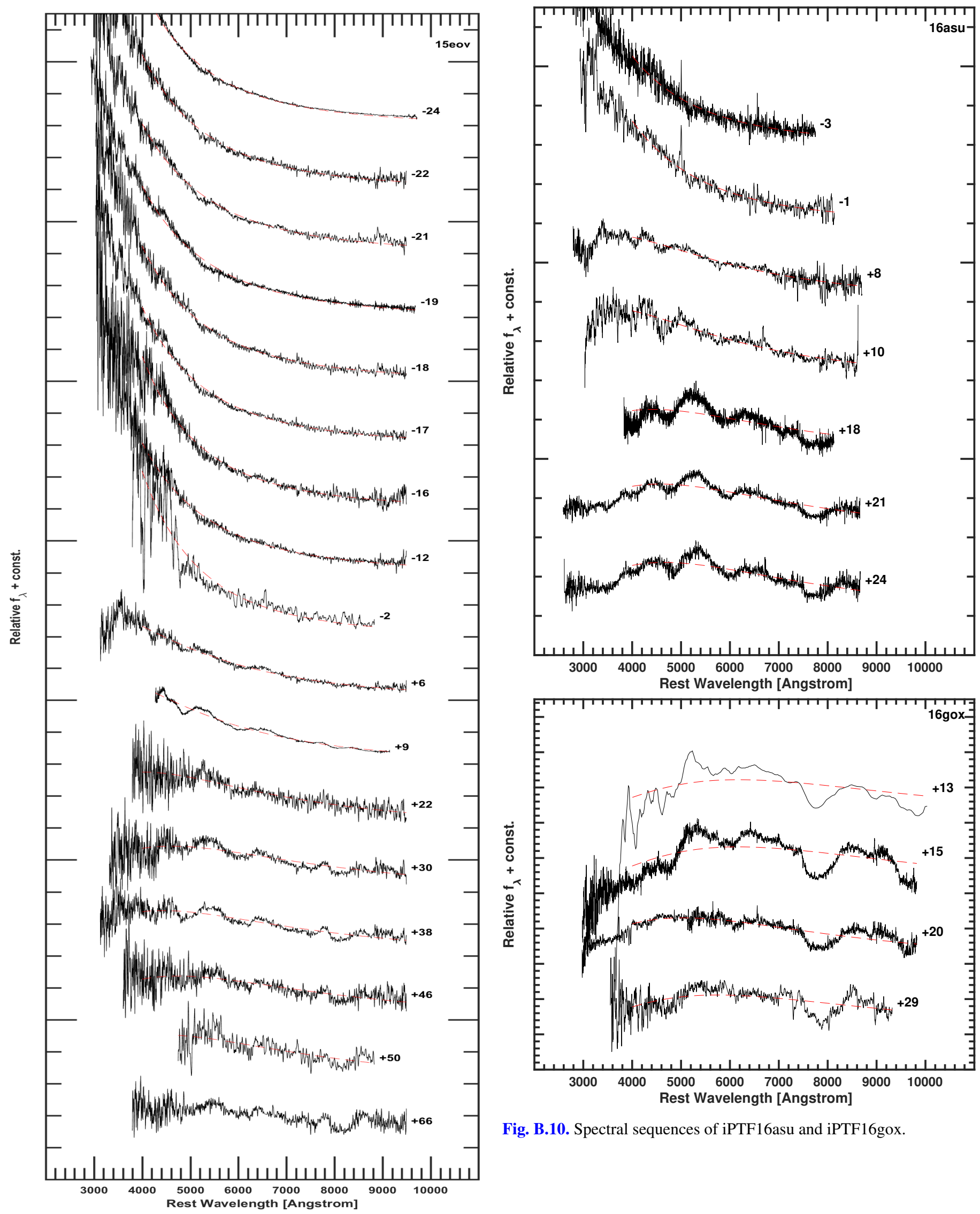

Fig. B.10. Spectral sequences of iPTF16asu and iPTF16gox.

Fig. B.9. Spectral sequence of iPTF15eov. 

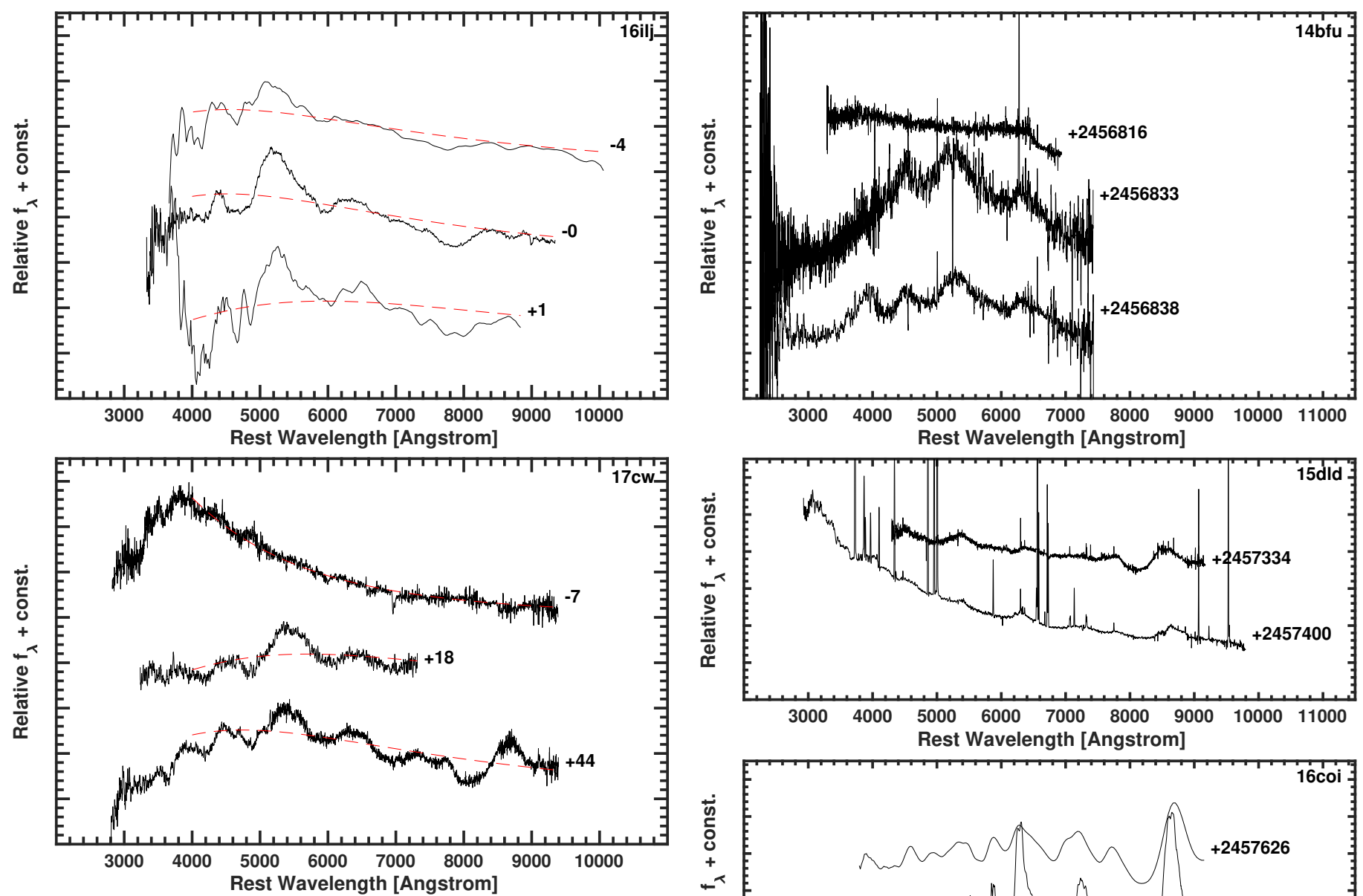

Fig. B.11. Spectral sequences of iPTF16ilj and iPTF17cw.
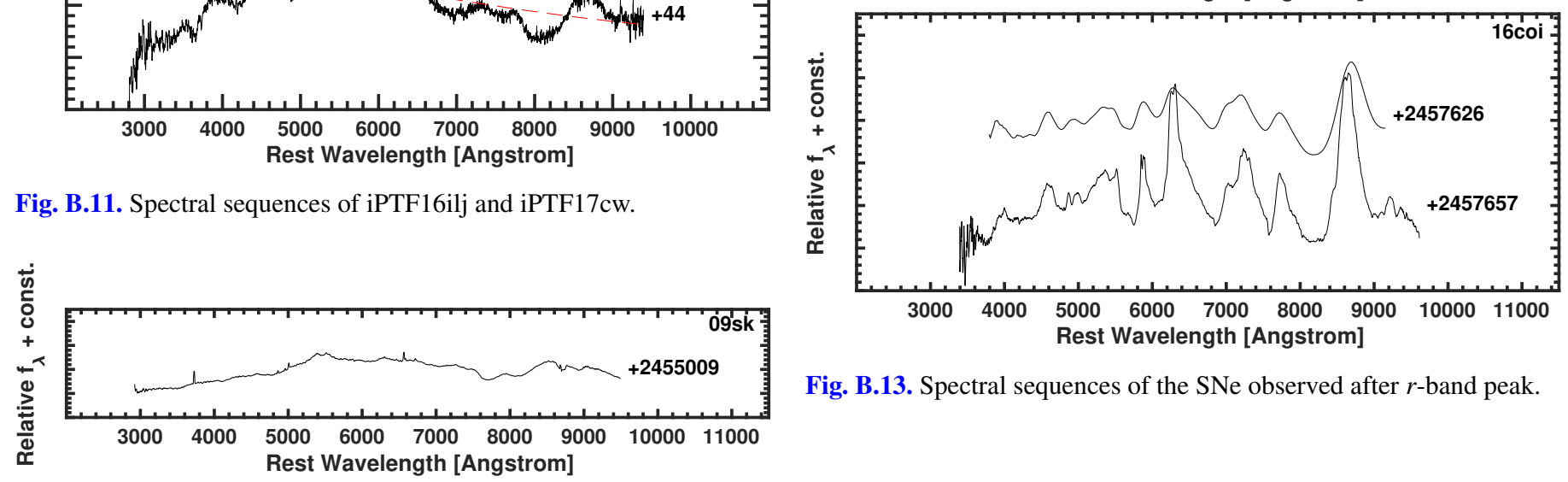

Fig. B.13. Spectral sequences of the $\mathrm{SNe}$ observed after $r$-band peak.
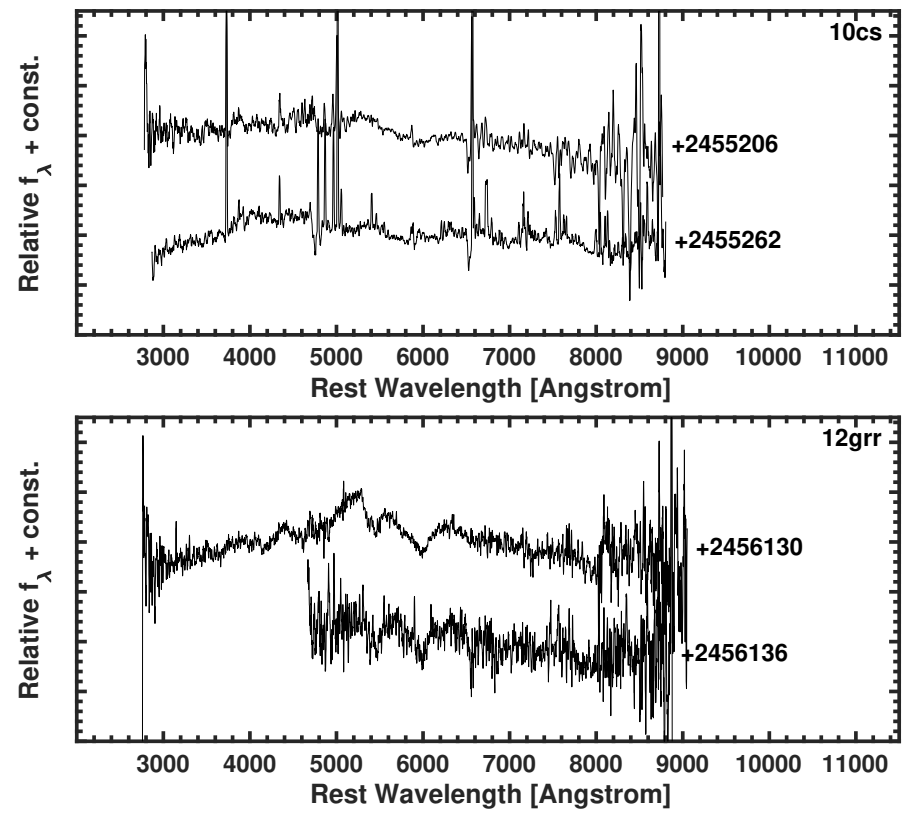

Fig. B.12. Spectral sequences of the SNe observed after $r$-band peak. 